\title{
Assessment and management of polycystic ovary syndrome: summary of an evidence-based guideline
}
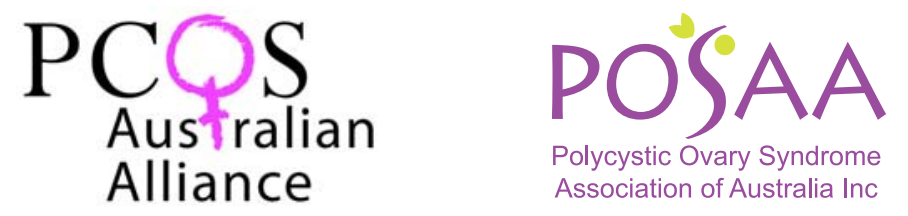

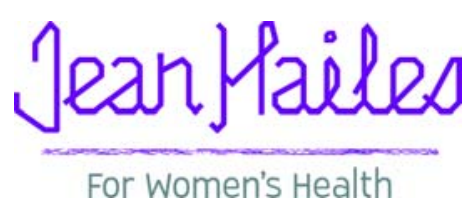

For Women's Health 


\section{AUTHORS}

Name

Professor Helena J Teede

Discipline

Endocrinology, Research, Public Health

Dr Marie L Misso

Research

Dr Amanda A Deeks

Counselling and Research Psychology

Dr Lisa J Moran

Professor Bronwyn GA Stuckey

Dr Jennifer LA Wong

Professor Robert J Norman

Dr Michael F Costello
Dietetics, Research

Endocrinology, Research

Endocrinology, Research

Obstetrics and Gynaecology, Reproductive Endocrinology, Research

Obstetrics and Gynaecology, Reproductive Endocrinology

\section{Organisational affiliation}

Director of Research, Jean Hailes Foundation for Women's Health Professor of Women's Health and Site Director, School of Public Health and Preventive Medicine, Monash University; Head of Diabetes Southern Health, VIC

Head of Evidence Synthesis Research Program, Jean Hailes Foundation for Women's Health; School of Public Health and Preventive Medicine, Monash University, VIC

Manager, Translation Services, Jean Hailes Foundation for Women's Health, VIC

Research Fellow, The Robinson Institute, University of Adelaide, SA

Medical Director, Keogh Institute for Medical Research;

Endocrinologist, Sir Charles Gairdner Hospital; Clinical Professor,

School of Medicine and Pharmacology, University of Western

Australia, WA

Deputy Head of Diabetes, Researcher, Southern Health, VIC

Director, The Robinson Institute, University of Adelaide, SA; Professor of Reproductive and Periconceptual Medicine, University of Adelaide, SA

Senior Lecturer in Obstetrics and Gynaecology, University of New South Wales; Clinical Academic, Reproductive Medicine, Royal Hospital for Women, NSW; Consultant, IVF Australia, NSW

\section{Contributing Authors}

\section{ASSESSMENT AND DIAGNOSIS GUIDELINE DEVELOPMENT GROUP}

\section{Name}

Professor Bronwyn Stuckey (Chair)

Dr Jacqueline Boyle

Associate Professor Elizabeth Davis

Ms Linda Downes

Associate Professor John Eden

Dr Meredith Frearson

Mrs Debbie Hester

Dr Ee Mun Lim

Ms Angela Melder

Dr Marie Misso

Professor Helena Teede

\section{Designation}

\section{Endocrinologist, Researcher}

Obstetrician, Gynaecologist,

Reproductive Endocrinologist, Researcher

Paediatric Endocrinologist

Project Manager

Reproductive Endocrinologist, Researcher

General Practitioner

Consumer

Chemical Pathologist, Endocrinologist

Senior Consultant in Clinical Effectiveness

Senior Evidence Officer

Endocrinologist, Researcher in Public Health, Project Director

\section{Organisation}

Keogh Institute for Medical Research, WA

Menzies School of Health, NT

Princess Margaret Hospital for Children, WA

Jean Hailes Foundation for Women's Health, VIC

Women's Health and Research Institute of Australia, NSW

Adelaide Health Care, SA

Polycystic Ovary Syndrome Association of Australia, NSW

PathWest Laboratory Medicine WA, WA

Centre for Clinical Effectiveness, Southern Health, VIC

Jean Hailes Foundation for Women's Health, VIC

Jean Hailes Foundation for Women's Health, VIC

\section{EMOTIONAL WELLBEING GUIDELINE DEVELOPMENT GROUP}

\section{Name}

Dr Amanda Deeks (Chair)

Ms Irene Apostolopoulos

Dr Leah Brennan

Professor David Clarke

Ms Linda Downes

Ms Marie Garrubba

Dr Cate Lombard

Ms Angela Melder

Professor Bronwyn Stuckey

Professor Helena Teede

Dr Mala Thondan

Associate Professor Beverley Vollenhoven

Dr Jennifer Wong

\section{Designation}

Counselling and Research Psychologist, Translation Manager

Consumer

Clinical and Health Psychologist

Psychiatrist, Researcher

Project Manager

Senior Evidence Officer

Dietitian, Researcher

Senior Consultant in Clinical Effectiveness

Endocrinologist, Researcher

Endocrinologist, Researcher in Public Health, Project Director

General Practitioner

Obstetrician, Gynaecologist

Endocrinologist, Service Development

\section{Organisation}

Jean Hailes Foundation for Women's Health, VIC

Polycystic Ovary Syndrome Association of Australia, NSW School of Psychology and Psychiatry, Monash University, VIC School of Psychology and Psychiatry, Monash University, VIC Jean Hailes Foundation for Women's Health, VIC

Centre for Clinical Effectiveness, Southern Health, VIC Jean Hailes Foundation for Women's Health, VIC Centre for Clinical Effectiveness, Southern Health, VIC Keogh Institute for Medical Research, WA Jean Hailes Foundation for Women's Health, VIC

Harp Family Medical, VIC

Monash IVF, VIC

Southern Health, VIC 


\section{THERAPY FOR INFERTILITY GUIDELINE DEVELOPMENT GROUP}

\section{Name}

Dr Michael F Costello (Chair)

Ms Irene Apostolopoulos

Ms Linda Downes

Professor Roger Hart

Ms Angela Melder

Dr Marie Misso

Associate Professor John Moss

Professor Robert Norman

Associate Professor Luk Rombauts

Professor Helena Teede

Ms Narelle Thredgold

Dr Jennifer Wong

\section{Designation}

Obstetrician, Gynaecologist, Reproductive Endocrinologist

Consumer

Project Manager

Obstetrician, Gynaecologist, Reproductive Endocrinologist

Senior Consultant in Clinical Effectiveness

Senior Evidence Officer

Health Economist

Obstetrician, Gynaecologist, Reproductive Endocrinologist, Researcher

Obstetrician, Gynaecologist, Reproductive Endocrinologist, Researcher

Endocrinologist, Researcher in Public Health, Project Director

Consumer

Endocrinologist

\section{Organisation}

School of Women's and Children's Health, University of New South Wales, NSW

Polycystic Ovary Syndrome Association of Australia, NSW

Jean Hailes Foundation for Women's Health, VIC

School of Women's and Infant's Health, University of Western Australia, WA

Centre for Clinical Effectiveness, Southern Health, VIC Jean Hailes Foundation for Women's Health, VIC

School of Population Health and Clinical Practice, University of Adelaide, SA

The Robinson Institute, University of Adelaide, SA

Department of Obstetrics and Gynaecology, Monash University, VIC

Jean Hailes Foundation for Women's Health, VIC

Polycystic Ovary Syndrome Association of Australia, NSW

Southern Health, VIC

\section{LIFESTYLE GUIDELINE DEVELOPMENT GROUP}

\section{Name}

Dr Lisa Moran (Chair)

Ms Irene Apostolopoulos

Ms Linda Downes

Dr Meredith Frearson

Dr Henry Ko

Dr Kate Marsh

Ms Angela Melder

Associate Professor Manny Noakes

Dr Nigel Stepto

Dr Mac Talbot

Professor Helena Teede

Dr Mala Thondan

\section{Designation}

Dietitian, Researcher

Consumer

Project Manager

General Practitioner

Senior Evidence Officer

Dietitian and Diabetes Educator

Senior Consultant in Clinical Effectiveness

Dietitian, Researcher

Exercise Physiologist, Researcher

Obstetrician, Gynaecologist

Endocrinologist, Researcher in Public Health, Project Director

General Practitioner

\section{Organisation}

The Robinson Institute, University of Adelaide, SA

Polycystic Ovary Syndrome Association of Australia, NSW

Jean Hailes Foundation for Women's Health, VIC

Adelaide Health Care, SA

Centre for Clinical Effectiveness, Southern Health, VIC Northside Nutrition and Dietetics, NSW

Centre for Clinical Effectiveness, Southern Health, VIC CSIRO Food and Nutritional Sciences, SA

School of Sport and Exercise Science and Institute of Sport Exercise and Active Living, Victoria University, VIC

Monash IVF, VIC

Jean Hailes Foundation for Women's Health, VIC

Harp Family Medical, VIC

\section{CO-OPTED EXPERTISE IN BARIATRIC SURGERY}

\section{Name}

Associate Professor Wendy Brown Associate Professor John Dixon Associate Professor Boyd Strauss Dr Amanda Vincent
Designation

Surgeon

Nutrition Specialist Physician, Researcher Nutrition Specialist Physician, Researcher Endocrinologist, Researcher

\section{Organisation}

Centre for Obesity Research and Education, Monash University, VIC Baker IDI Heart and Diabetes Institute, VIC Body Composition Laboratory, Southern Health, VIC Jean Hailes Foundation for Women's Health, VIC 


\section{CONTENTS}

Assessment and management of polycystic ovary syndrome: summary of an evidence-based guideline

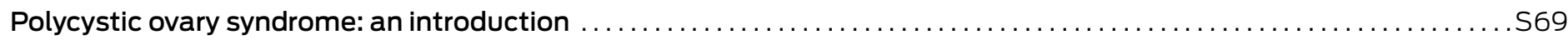

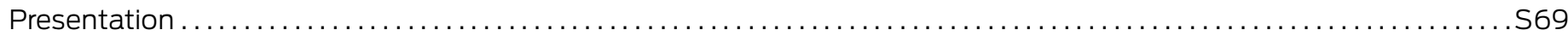

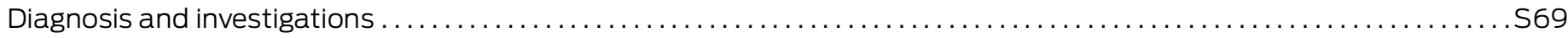

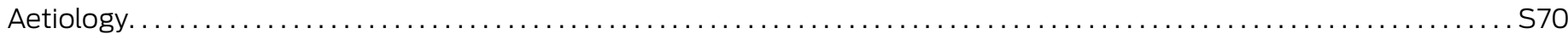

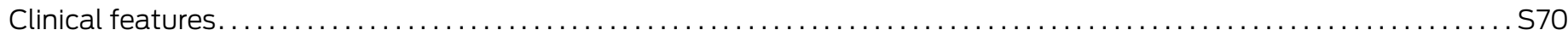

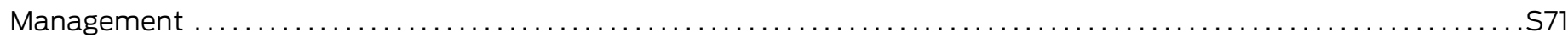

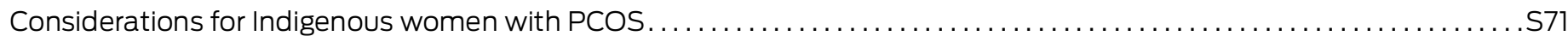

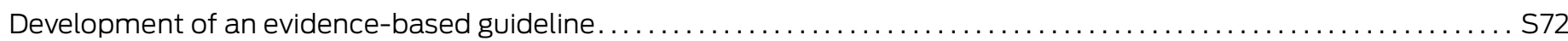

Summary of the evidence-based guideline

\section{Section 1.}

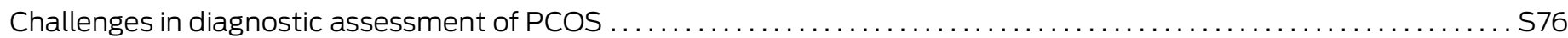

\section{Section 2.}

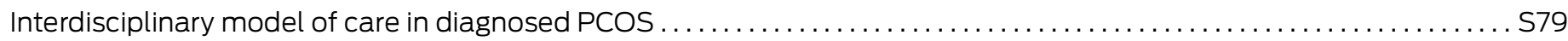

\section{Section 3.}

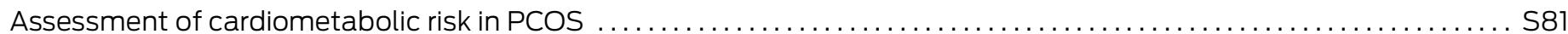

\section{Section 4.}

Assessment of emotional wellbeing in PCOS

\section{Section 5 .}

Lifestyle management in PCOS

\section{Section 6.}

Non-pharmacological first-line management of infertility in PCOS

\section{Section 7.}

Pharmacological management of infertility in PCOS

\section{Section 8.}

Surgical management of infertility in PCOS

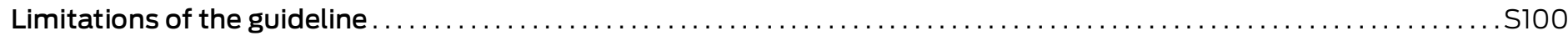

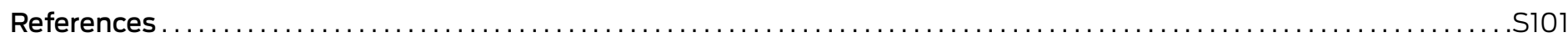

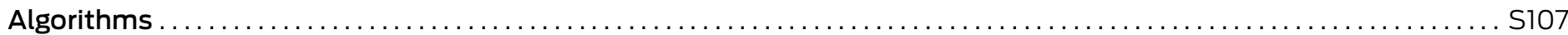




\section{Assessment and management of polycystic ovary syndrome: summary of an evidence-based guideline}

\section{Polycystic ovary syndrome: an introduction}

Polycystic ovary syndrome (PCOS) has recently been shown to affect a striking $12 \%-21 \%$ of Australian reproductive-age women, being more common among those who are overweight or of Indigenous background. ${ }^{1}$ PCOS can be a frustrating experience for women, a complex syndrome for clinicians and a scientific challenge for researchers, and is a major public health concern.

Although reproductive features are prominent, PCOS has potential for major metabolic consequences, including obesity and related type 2 diabetes mellitus (DM2) as well as cardiovascular disease (CVD), all of which are currently national health priority areas. ${ }^{2,3}$ It also has significant mental health and psychological impact, impairing quality of life (QoL). ${ }^{4,5}$ Because increased obesity exacerbates incidence, prevalence and severity of PCOS, and weight loss improves reproductive, metabolic and psychological features, lifestyle change should be first-line therapy for PCOS. $^{6}$

Helena J Teede
Marie L Misso
Amanda A Deeks
Lisa J Moran
Bronwyn G A Stuckey
Jennifer L A Wong
Robert J Norman
Michael F Costello
on behalf of
the Guideline
Development
Groups
helena.teede@
monash.edu
doi:10.5694/mjal1.10915

Figure 1. The aetiological, hormonal and clinical features of polycystic ovary syndrome

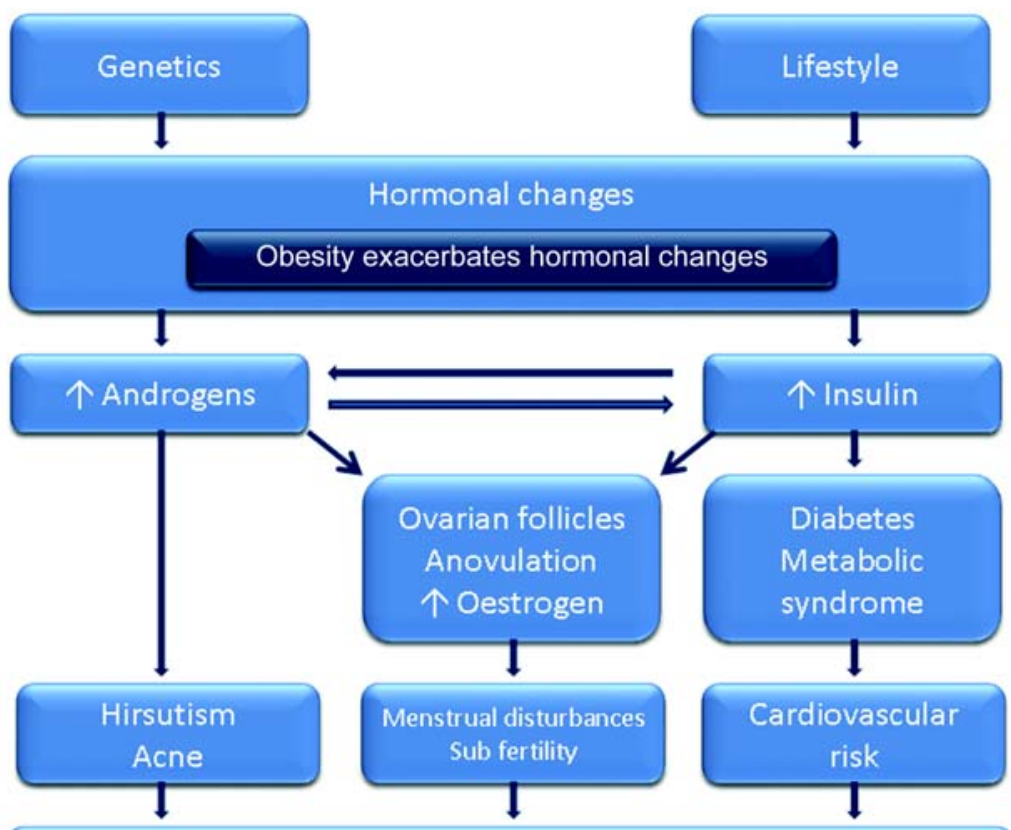

Psychosocial issues: body image, self esteem, depression, anxiety

Adapted and reproduced from Teede at al with permission from the Royal Australian College of General Practitioners. ${ }^{13}$

It is estimated that $70 \%$ of Australian women with PCOS remain undiagnosed; ${ }^{1}$ clinical practice is inconsistent; ${ }^{7}$ psychological issues are under-recognised; ${ }^{5}$ and there is little focus on lifestyle and prevention, with most services targeting infertility and costly assisted reproductive technology. Given the prevalence, disease burden, health costs and clear gaps in care, PCOS is highlighted in national policy and has been prioritised by government, with funding for development of a national PCOS evidence-based guideline and translation of evidence into practice. Here, we present a brief general clinical introduction to PCOS, concise guideline algorithms and clinical pathways and a comprehensive summary of the Evidence based guideline for assessment and management of PCOS (available at http:// www.managingpcos.org.au/pcos-evidence-basedguidelines). ${ }^{8}$

\section{Presentation}

PCOS has significant and diverse implications, including reproductive (hyperandrogenism, hirsutism, anovulation, infertility), metabolic (insulin resistance [IR], impaired glucose tolerance, DM2, adverse cardiovascular risk profiles) and psychological features (increased anxiety and depression and worsened QoL). ${ }^{9}$ Presentation varies across the life span. Hyperandrogenic features are often most prominent among adolescents, ${ }^{10}$ fertility issues most prominent among women in their 20s and 30s, and metabolic challenges most notable after this. ${ }^{11}$ The propensity to weight gain and psychological challenges affect all ages, and metabolic features can occur early, especially among those who are overweight. Variations across ethnic groups should also be noted, such as fewer hyperandrogenic dermatological features and more severe metabolic features in Asian women, even without weight gain. Indigenous women appear to have a higher prevalence and severity of PCOS..$^{1,12}$ The clinical presentation of PCOS is outlined in Figure 1.

\section{Diagnosis and investigations}

Diagnosis of PCOS is now largely based on the Rotterdam criteria, $^{14}$ which are inclusive of the original National Institutes of Health (NIH) criteria ${ }^{15}$ and require two of three key features: oligo- or anovulation, clinical and/or biochemical hyperandrogenism and polycystic ovaries on ultrasound (Figure 2). However, as noted, PCOS phenotypes vary widely depending on life stage, genotype, ethnicity and environmental factors, including lifestyle and body weight. 


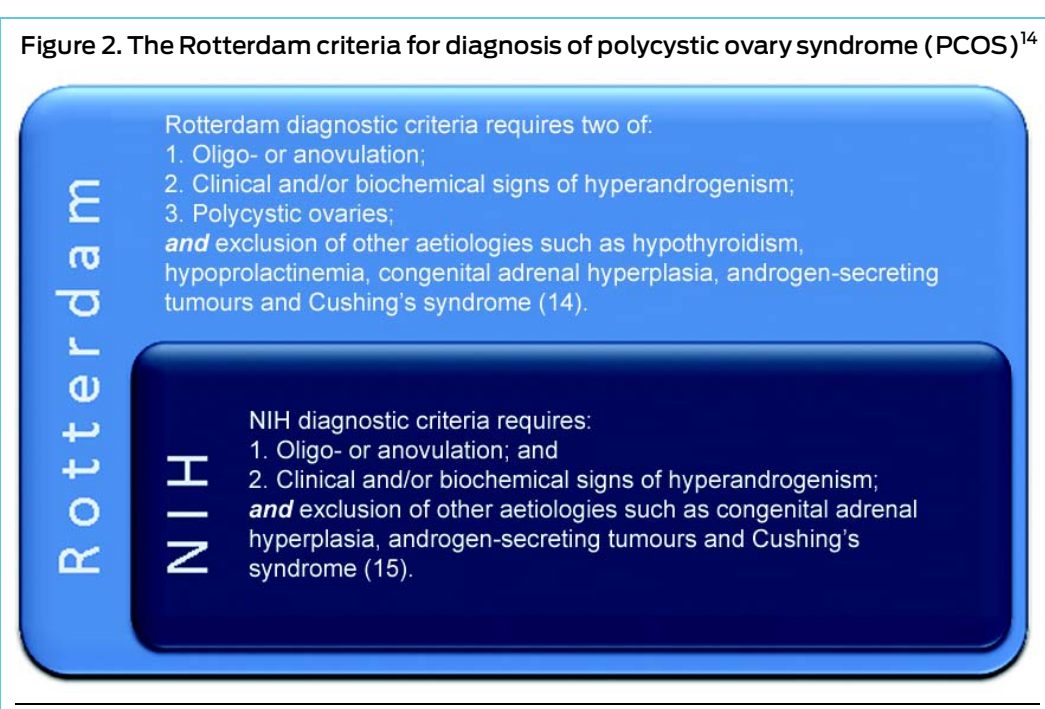

The Rotterdam criteria are inclusive of National Institutes of Health $(\mathrm{NIH})$ criteria in that a woman diagnosed with PCOS using the NIH criteria will also meet Rotterdam criteria; however, a woman diagnosed with PCOS using Rotterdam criteria may not meet NIH criteria.

Diagnostic investigations must exclude other causes and include thyroid function tests and prolactin and folliclestimulating hormone (FSH) levels. ${ }^{9}$ For diagnosis, androgen levels should be measured; however, optimal methodology remains very controversial and is addressed in Section 1. Vaginal ultrasound is often needed for diagnosis where hyperandrogenism and anovulation are not both clearly present. Ultrasound can check for polycystic ovaries and endometrial thickness. However, vaginal ultrasound should be reserved for sexually active women. The role of ultrasound remains controversial for adolescents, among whom a polycystic appearance of the ovaries is very common, potentially leading to overdiagnosis; ${ }^{16}$ hence, this area is also covered in the guideline. Other diagnostic investigations are based on clinical discretion.

Screening is also vital to detect PCOS complications and guide prevention and treatment. Comprehensive cardiovascular risk-factor screening, including family history, ethnic group, body mass index (BMI), waist circumference, smoking status, blood pressure, glycaemic status (oral glucose tolerance test [OGTT]) and lipid profile, is important at diagnosis and should be repeated with a frequency informed by metabolic risk (eg, body weight, age, family history, ethnicity) as outlined in Section 3. Optimal methodology for the routine screening for prediabetes and DM2 has been controversial in PCOS, but because lifestyle change and metformin improve IR in PCOS, and among other at-risk groups these measures have been shown to dramatically reduce progression to diabetes, early detection, including detection of prediabetes, is vital and is addressed in the guideline.

\section{Aetiology}

PCOS is an endocrine disorder, the pathophysiology of which remains unclear. Genetic and environmental contributors combine with obesity, ovarian dysfunction and hormonal drivers to contribute to the aetiology of
PCOS. ${ }^{17,18}$ The underlying hormonal imbalance may include a combination of increased androgens and/or hyperinsulinaemia secondary to IR (Figure 1). Greater understanding of cause has been hampered by a lack of ideal methods to assess either hyperandrogenism or IR. Hyperandrogenism is detected in around $60 \%-80 \%$ of women with PCOS, and IR is a pathophysiological contributor in around $50 \%-80 \% .{ }^{19}$ Obesity increases reproductive features - hyperandrogenism, hirsutism, infertility and pregnancy complications - both independently and by exacerbating PCOS. ${ }^{20,21}$ Furthermore, obesity exacerbates the PCOS-related increased risk factors for impaired glucose tolerance, DM2 and $\mathrm{CVD}^{22}$ while obesity also affects psychological features of PCOS.

\section{Clinical features}

PCOS is a chronic condition that manifests across the life course. Women with PCOS present with psychological, 5,23 reproductive $^{24}$ and metabolic implications. In terms of psychosocial implications, challenges to feminine identity and body image due to obesity, acne, excess hair, infertility and long-term health-related concerns compromise QoL and adversely affect mood and psychological wellbeing. With a higher prevalence and greater severity of depression and anxiety, low self-esteem, negative body image, and psychosexual dysfunction, ${ }^{5,25}$ assessment of psychological functioning in women with PCOS is vital. This is relevant to clinical care as mood disturbance, in turn, impairs QoL and adversely affects ability to selfmanage and optimise lifestyle. Optimal approaches to screening and assessment of psychological functioning in PCOS are unknown and recognition is generally poor; hence, this area was prioritised in the guideline (Section 4). If mood disturbance is detected during screening, further assessment and management is required.

Reproductive and reproductive hormonal features are often the best-recognised features in PCOS as they form the basis of the diagnostic criteria. ${ }^{14}$ These include clinical and biochemical hyperandrogenism, anovulation, subfertility and polycystic ovaries on ultrasound. A key point is that fertility is not necessarily impaired in all PCOS cases - some women conceive without medical intervention, depending on the severity of the condition. Age and BMI have a critical role in infertility risk in PCOS; therefore, early family initiation (before the age of 30-35 years) combined with maintaining a BMI $<31 \mathrm{~kg} / \mathrm{m}^{2}$ is ideal for increasing the chance of conceiving.

Metabolic features of PCOS include an apparent propensity for excess weight gain, an increased prevalence of prediabetes and DM2, a 5-10-fold risk of progression from prediabetes to DM2 and a 4-7-fold risk of DM2. ${ }^{11}$ Cardiovascular risk factors are increased and CVD appears more prevalent among women with PCOS despite inadequate long-term studies to appropriately address this question. $^{22}$ In the general population, IR is a predictor of CVD. ${ }^{26,27}$ Women with PCOS also have an increased prevalence of metabolic syndrome (associated with an increased risk for DM2 and CVD) ${ }^{28}$ individual risk factors for CVD and clinical signs of atherosclerosis, ${ }^{29,30}$ which are all exacerbated by obesity. Women with PCOS are 
therefore a population at high risk of developing DM2 and CVD. As DM2 and subsequent CVD are the primary cause of death in Australian women, any increase in prevalence will have significant public health implications. It is also important to note that relatives of women with PCOS may have increased risk of diabetes and increased CVD risk factors. Metabolic features are often poorly appreciated in PCOS; hence, recommendations on screening and assessment of DM2 and CVD risk factors are covered in the guideline.

Obesity or excess weight is a major cause of chronic disease in Western countries. In Australia, $56 \%$ of the adult population is overweight (BMI $\geqslant 25 \mathrm{~kg} / \mathrm{m}^{2}$ ) or obese (BMI $\geqslant 30 \mathrm{~kg} / \mathrm{m}^{2}$ ). In $2007,31 \%$ of women were overweight and $24 \%$ of women were obese. Recent data from the Australian Longitudinal Study on Women's Health showed that among 26-31-year-old women, 20.4\% were overweight and a further $13.9 \%$ were obese. ${ }^{31}$ Overall, the proportion of adults who are obese has doubled in the past 20 years. ${ }^{32}$ Obesity is now the primary cause of chronic disease among Australian women, with adverse outcomes including DM2 and CVD. ${ }^{31}$ Obesity has a specific impact on women's reproductive health, increasing the prevalence and severity of PCOS, infertility, pregnancy complications, gestational diabetes and fetal pregnancy complications, with substantial and escalating economic costs. ${ }^{33,34}$ Indeed, the adverse impact of obesity on fertility, exacerbated by delay in childbearing, is resulting in a significant social, health and economic burden in Australia. ${ }^{35}$ Given the dramatic increase in obesity, the guideline addresses weight loss and prevention of weight gain through lifestyle intervention (Section 5).

\section{Management}

Therapy should focus on both the short- and long-term reproductive, metabolic and psychological features. It is important to address psychological factors initially to optimise self-efficacy, readiness to change and sustainability of lifestyle interventions as well as to improve QoL. Screening, assessment and treatment of depression and anxiety are vital, and recognition of other aspects of emotional wellbeing, including poor body image, sexual dysfunction, disordered eating and eating disorders - all more common among women with PCOS — is important for improving QoL. Optimal approaches to screening and assessment of emotional wellbeing among women with PCOS remain unclear and are also addressed in this guideline (Section 4). Once recognised, poor emotional wellbeing and mood disorders should be addressed to improve QoL among women with PCOS.

PCOS management should focus on support and education, and needs to strongly emphasise healthy lifestyle, with targeted medical therapy as required. Given the putative aetiological role of IR and obesity in PCOS, prevention of weight gain is important across the life span. Furthermore, among those who are already overweight, multidisciplinary lifestyle intervention aimed at improving IR and aiding weight management is recognised as firstline therapy for most women who are overweight. ${ }^{6}$ Modest weight loss of $5 \%-10 \%$ of initial body weight significantly reduces IR and has been demonstrated to ameliorate many of the features of PCOS. ${ }^{6}$ Optimal methods for achieving weight loss and prevention of weight gain remain unclear and are a focus of this guideline (Section 5).

Short-term diets rarely lead to permanent weight loss, and lifestyle change requires behavioural change. Healthcoaching principles can be incorporated to optimise readiness to change, and include education and accurate risk perception, which can assist with motivation through education and tailoring the knowledge relevant to the individual. Once ready to change, support is needed to convert this to action with effective strategies including patient-driven goal setting (eg, $5 \%$ of body weight loss, small improvements in exercise), so that these incremental changes are seen as achievements. Multidisciplinary involvement in care is often useful in the early stages to support education and behaviour change and is explored further in this guideline (Section 2).

In addition to lifestyle measures, therapy in PCOS can be targeted to specific clinical presentations. Although there is a plethora of options for therapy in PCOS, in this guideline we have focused on the most controversial interventions, where little guidance is currently available. Assessment of mood disorders and emotional wellbeing is prioritised in the guideline, yet treatment is well guided by a range of existing clinical guidance tools. ${ }^{36-43}$ Hirsutism treatment is also guided by a recent and comprehensive international statement, ${ }^{44}$ so these areas are not covered in the guideline. Infertility remains a highly controversial area and is covered in detail in the guideline (Sections 6, 7 and 8). DM2 and CVD risk assessment is included (Section 3); yet treatments for these established complications are covered in other specific national evidenced-based guidelines, ${ }^{45,46}$ and have not been reproduced here. Optimal therapy for infertility is one of the most controversial areas of PCOS management and includes lifestyle interventions, medical and surgical ovulation induction, consideration of bariatric surgery for preconception weight loss, and in-vitro fertilisation (IVF). All these areas are covered in the guideline except for IVF therapy, as this was deemed to be of a lower priority than the first-line lifestyle measures and ovulation induction therapies. Potential targeted treatment options for PCOS are summarised in Box 1.

\section{Considerations for Indigenous women with PCOS}

The prevalence of PCOS among Indigenous Australian women appears to be as high as $21 \%$ by the Rotterdam criteria $^{12}$ and the NIH criteria, ${ }^{50}$ and increases with rising BMI. $^{12}$ In a group of Indigenous women with PCOS, $30.3 \%$ were obese and $7.0 \%$ had a normal BMI. ${ }^{12}$

DM2 and obesity are associated with major morbidity among Indigenous women. The National Aboriginal and Torres Strait Islander Health Survey (NATSIHS) found that Indigenous Australians are 1.2 times more likely to be overweight or obese than non-Indigenous Australians, and this disparity is greatest for women. ${ }^{51}$ DM2 is the second-commonest cause of mortality and disabilityadjusted life-years (DALY) among Indigenous women. ${ }^{52}$ The DALY rate ratios (age-standardised to total Indigenous population) for ischaemic heart disease and DM2 among Indigenous women, compared with all Australian women, 
Box 1. Summary of potential targeted treatment options for polycystic ovary syndrome (PCOS)

\section{Oligomenorrhoea/amenorrhoea}

- Lifestyle change (5\%-10\% weight loss + structured exercise)

- Oral contraceptive pill (OCP) (low oestrogen doses [eg, 20 $\mu \mathrm{g}$ ] may have less impact on insulin resistance $)^{47}$

- Cyclic progestins (eg, 10 mg medroxyprogesterone acetate 10-14 days every 2-3 months)

- Metformin (improves ovulation and menstral cyclicity)

\section{Hirsutism}

Choice of options depends on patient preferences; impact on wellbeing; and access and affordability: ${ }^{44}$

- Self-administered and professional cosmetic therapy are first line (laser recommended)

- Eflornithine cream can be added and may induce a more rapid response

- If cosmetic therapy is not adequate, pharmacological therapy can be considered

- Pharmacological therapy

$>$ Medical therapy if patient is concerned and cosmetic therapy is ineffective/ inaccessible/unaffordable

$>$ Primary therapy is the OCP (monitor glucose tolerance in those at risk of diabetes)

$>$ Anti-androgen monotherapy (eg, spironolactone or cyproterone acetate) should not be used without adequate contraception

$>$ Trial therapies for $\geqslant 6$ months before changing dose or medication

$>$ Combination therapy - if $\geqslant 6$ months of OCP is ineffective, add anti-androgen to OCP (twice daily spironolactone $>50 \mathrm{mg}$ or cyproterone acetate $25 \mathrm{mg} /$ day, days 1-10 of OCP)

\section{Infertility}

- Lifestyle intervention (to optimise preconception health and fertility and reduce pregnancy and long-term complications)

- Advise on folate, smoking cessation and optimal weight and exercise before conception

- Given age-related infertility, advise women to optimise family initiation

- Infertility therapies may include clomiphene citrate, metformin, gonadotrophins, surgery and in-vitro fertilisation.

\section{Cardiometabolic risk}

- Lifestyle change: $>5 \%$ weight loss in those who are overweight reduces diabetes risk by approximately $50 \%-60 \%$ in high-risk groups ${ }^{48}$

- Optimise cardiovascular risk factors

- Consider metformin* (reduces the risk of diabetes by 50\% in adherent high-risk groups $)^{48}$

Adapted and reproduced with permission from Teede et $\mathrm{al},{ }^{9}$ not generated directly from the evidence-based guidelines. Hirsutism therapy is summarised from existing hirsutism clinical practice guidelines. ${ }^{44} *$ Metformin and the OCP are not currently approved for use to manage PCOS by many regulatory bodies. The OCP is indicated for contraception and metformin for diabetes. However, their use is supported by evidence and is recommended by international and national specialist societies. ${ }^{49}$

are 6.6 and 6.3, respectively; and the mortality rate ratio is 5.0 for ischaemic heart disease and 18.9 for DM2. ${ }^{52}$

The risk of metabolic complications is already high among Indigenous women, independent of PCOS; therefore, PCOS can amplify metabolic risk in these women. Given that these metabolic complications are largely preventable, it is important to provide early access to care.

The leading cause of burden of disease among Indigenous women in the NATSIHS was anxiety and depression, accounting for $10 \%$ of the burden. ${ }^{51}$ Little is known about the prevalence of eating disorders and disordered eating among Indigenous women. Social and cultural factors influence emotional wellbeing, and the challenges facing many Indigenous women are likely to amplify the impact of PCOS on emotional wellbeing. Further research in this area is needed.

Access to culturally appropriate care, services and programs is currently not optimal. Access issues are a key barrier for many Indigenous women, as health services generally, and women's health services in particular, are limited in rural and remote locations. There are many barriers to healthy lifestyles, including the high cost of maintaining a healthy diet in rural and remote locations. Socioeconomic factors, such as poverty and overcrowding, make the use of refrigerators and kitchen equipment to cook healthy food difficult. Lifestyle programs may need to be applied in different ways to engage Indigenous women and incorporate exercise into daily activities, especially in rural and remote locations, due to a lack of service provision and facilities.

Other issues for Indigenous women include that the role of ultrasound in the Indigenous setting is questionable due to limitations in care and access to ultrasound facilities and service provision in rural and remote locations; and there may be cultural factors and potential issues around acceptability of bariatric surgery.

It is important to encourage and enable Indigenous women with PCOS to access services that are available and address potential barriers presented by cultural and traditional health practices. Work is currently underway to adapt, translate and implement the recommendations outlined here to Indigenous settings.

\section{Development of an evidence-based guideline}

\section{Rationale and methods}

Given its heterogeneous clinical features across the life span, PCOS is a condition that engages many health disciplines. The associated complications are serious yet are often largely preventable; however, there is a lack of awareness of PCOS among consumers and health professionals. It is essential that consumers and health professionals recognise the life-course implications of PCOS, identify the early signs and symptoms and work together to manage PCOS and prevent its complications - especially as the burden and cost of PCOS complications, including infertility, DM2, CVD and emotional wellbeing issues are significant.

Currently, there is limited consensus among different medical specialties as to the optimal management of PCOS in Australia. ${ }^{7,53}$ Diagnosis and treatment of PCOS can therefore differ depending on the health professional consulted (eg, general practitioner, endocrinologist or gynaecologist). ${ }^{7}$ There are limited clinical guidelines and no evidence-based guidelines, either in Australia or internationally, for assessment or management of women with PCOS; rather, PCOS is briefly mentioned within guidelines for the management of obesity and DM2. ${ }^{45,54}$ Where international clinical guidelines for the assessment and management of women with PCOS exist, they are informed by expertise and, in some cases, evidence, but are not rigorously developed evidenced-based guidelines; they do not consider psychological issues; and they offer simplistic advice on lifestyle management of PCOS. There is no guidance on the assessment and management of PCOS among Indigenous women, nor any adaptation for the Australian context.

Overall, many areas of controversy remain in PCOS. Comprehensive evidence-based guidelines are warranted to optimise diagnosis, assessment and management. Accordingly, the Jean Hailes Foundation for Women's Health has facilitated the formation of an independent 


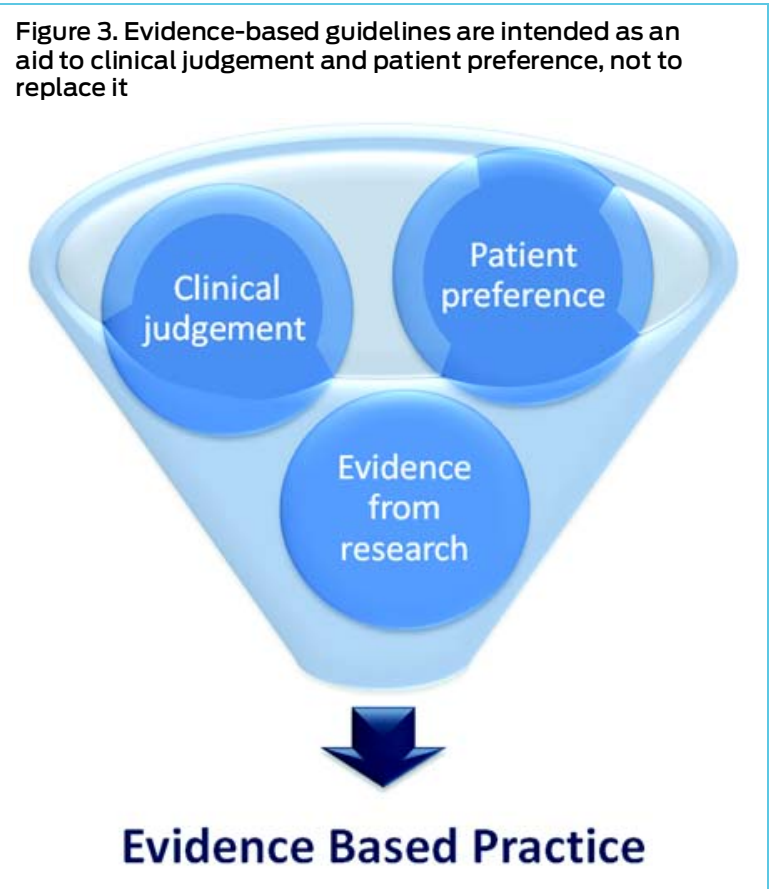

PCOS Australian Alliance, bringing together health professionals, researchers, consumers and policymakers to advance knowledge and quality of care in PCOS. The federal government has funded the PCOS Australian Alliance, under the auspices of the Jean Hailes Foundation, to produce national evidence-based guidelines. The full version of the guideline has been approved by the National Health and Medical Research Council (NHMRC) (for detail about obtaining NHMRC approval, please see the full guideline), is endorsed by the Royal Australian College of General Practitioners and is freely available at http:// www.managingpcos.org.au/pcos-evidence-basedguidelines. ${ }^{8}$ This is a summary version of the full guideline. The Jean Hailes Foundation was funded to translate the guideline into practice, including freely available independent evidence-based information on PCOS for health professionals and women at http:// www.managingpcos.org.au.

\section{Scope}

The purpose of the guideline is to integrate the best available evidence with clinical expertise and consumer preferences; to provide health professionals, consumers and policymakers with guidance on timely diagnosis, accurate assessment and optimal management of PCOS; and to promote consistency of care and prevention of complications in primary care and specialist settings.

The guideline is relevant to the assessment and management of reproductive-age adolescents and women with PCOS, including women with PCOS who are experiencing infertility. The guideline will apply in all health care settings and to a broad audience, including: community care practitioners; Indigenous health care workers; GPs; nurses; endocrinologists; obstetricians and gynaecologists; allied health professionals psychologists, dietitians, exercise physiologists and physiotherapists; patients; community support groups (eg, the Polycystic Ovary Syndrome Association of Australia [POSAA]); the general public; students; and policymakers.

PCOS is a syndrome, and as such, no single diagnostic criterion is sufficient for diagnosis. The 2003 Rotterdam consensus workshop concluded that PCOS diagnosis requires at least two of: oligo- or anovulation, hyperandrogenism (clinical and/or biochemical) and polycystic ovaries on ultrasound (Figure 2). ${ }^{14}$ The evidence-based guideline development groups and the Alliance agreed to endorse the Rotterdam diagnostic criteria for the guideline, while recognising there are current limitations of all definitions.

\section{Methodology}

Guidelines are intended to improve patient outcomes, promote standardised care, develop standards to assess the clinical practice of health care professionals, and promote research and translation into practice. Guidelines are developed by drawing from clinician judgement, patient preference and research evidence, and are intended to aid clinical judgement and patient preference, not to replace it (Figure 3). The ultimate decision about clinical management of an individual patient will always depend on the clinical circumstances, patient preferences, and the clinical judgement of the health care team. Although there are many types of guidelines, this NHMRC-approved evidence-based guideline followed a rigorous, systematic process of development, which is briefly outlined below and in detail in the full guideline. ${ }^{8}$

An independent PCOS Australian Alliance was formed in 2008 after a national workshop facilitated by the Jean Hailes Foundation for Women's Health, which brought together key leaders from the research and multidisciplinary clinical sectors, with consumers providing a driving force through the peak national support group, POSAA. The vision of the Alliance is to improve the lives of Australian women with PCOS through education, research and evidence-based health care. One of the priorities of the Alliance was to develop an evidence-based guideline for PCOS.

This guideline was developed as outlined in the NHMRC standards and procedures for externally developed guidelines. ${ }^{55}$

The Alliance identified key clinical objectives for the guideline based on highest clinical priority, greatest knowledge gaps, factors identified by the Australian government (which funded the guideline), and the expertise of Alliance members. The identified key clinical priorities focused on care of women with PCOS to facilitate early diagnosis of PCOS; early detection and treatment of depression, anxiety and mood disorders; early detection and diagnosis of risk factors for prediabetes, DM2 and CVD; and early detection and treatment of fertility problems and prevention of pregnancy complications.

Multidisciplinary guideline development committees included a Project Board, PCOS Australia Alliance Strategic Advisory Group and four guideline development groups. Each guideline development group comprised a chair, professional group members with specific expertise 
Table 1. National Health and Medical Research Council grades for recommendations ${ }^{56}$

A Body of evidence can be trusted to guide practice.

B Body of evidence can be trusted to guide practice in most situations.

C Body of evidence provides some support for recommendation but care should be taken in its application.

D Body of evidence is weak and recommendation must be applied with caution.

in PCOS and the clinical area of interest (eg, psychologist in the emotional wellbeing guideline development group), a consumer representative from POSAA, evidence officers and, where possible, a representative to provide context for the Indigenous setting. Indigenous representation was present on the PCOS Australian Alliance Strategic Advisory Group, and the guideline development groups comprised clinicians with experience working with Indigenous communities.

These multidisciplinary groups determined and prioritised the clinical questions addressed in this guideline and developed the clinical practice and research recommendations from the evidence reviews. For more detail about the development and prioritisation of clinical questions, please see the full guideline. ${ }^{8}$

To facilitate this process using an evidence-based approach, the chairs of each guideline development group attended a 1-day workshop, facilitated by the Southern Health Centre for Clinical Effectiveness, where the methods of identifying, appraising and synthesising evidence; grading the strength of evidence and its suitability to support evidence-based recommendations; and the process of guideline development overall were described in detail.

Evidence reviews were conducted for each of the 22 identified clinical questions. Search strategies were developed according to a-priori selection criteria for each clinical question. Searches were limited to English language articles and there were no limits on year of publication. The literature was searched until November 2010. The following electronic databases were employed to identify relevant evidence: Australasian Medical Index, CINAHL, the Cochrane Library, the Cochrane Database of Systematic Reviews, DARE (Database of Abstracts of Reviews of Effects), the Cochrane Central Register of Controlled Trials, the Cochrane Database of Methodology Reviews, the Cochrane Methodology Register, Health Technology Assessment Database, the United Kingdom National Health Service Economic Evaluation Database, EMBASE, EBMR, MEDLINE and PsycINFO. Bibliographies of relevant studies identified by the search strategy and relevant reviews/meta-analyses were also searched. Included studies were classified according to the NHMRC levels of evidence ${ }^{56}$ and appraised using a-priori criteria according to study design, using a descriptive component approach to assign a risk of bias rating. ${ }^{57}$ In accordance with the selection criteria, data were extracted from included studies using a specially developed data extraction form, ${ }^{57}$ and meta-analyses were performed where appropriate. The guideline development groups were able to develop guideline recommendations from these evidence reviews. The evidence reviews for each question can be found in the supporting document to the full guideline: Evidence report: evidence based guidelines for assessment and management of PCOS, available at http:// www.managingpcos.org.au/pcos-evidence-basedguidelines.

The guideline contains 38 recommendations, each of which is assigned a grade. In developing the guideline recommendations, the guideline development groups placed emphasis on accurate assessment and management of PCOS. The recommendations in this guideline are strengthened by the use of rigorous methodology for evidence review and guideline development, including use of: study designs least susceptible to bias; a-priori criteria for inclusion and appraisal of studies; extraction of study data; and meta-analysis where appropriate. The recommendations were formulated using a considered judgement process that took into account the amount and quality of available evidence as well as its generalisability and applicability to current practice in Australia.

Each evidence-based recommendation was given an overall grading from $\mathrm{A}$ to $\mathrm{D}$, according to the NHMRC grades of recommendations for guideline developers (Table 1). ${ }^{56}$ Evidence grading is provided primarily to inform users about the strength of the evidence underpinning each recommendation. Where there was insufficient high-quality evidence in specific patient groups, lower-quality evidence or data from other patient groups, and where there was consensus among the guideline development group, combined with clinician and patient preferences, clinical consensus recommendations were developed. Clinical practice points have also been included, where important issues (such as safety, side effects or risks) arose from discussion of evidence-based or clinical consensus recommendations. Further points of relevance to the clinical implementation of recommendations were made in "implications of the recommendations" sections, including consideration of resource implications.

The words "should", "could" and "should not" do not directly reflect the grade or classification allocated to a recommendation, and are independent descriptors intended to reflect the judgement of the multidisciplinary guideline development group about the practical application of the recommendation, balancing benefits and harms. Where the word "should" is used in the recommendations, the guideline development group judged that the benefits of the recommendation (whether evidence-based or clinical consensus) clearly exceed the harms, and that the recommendation can be trusted to guide practice. Where the word "could" is used, either the quality of evidence was underpowered, or the available studies demonstrated little clear advantage of one approach over another, or the balance of benefits to harm was unclear. Where the words "should not" are used, there is either a lack of appropriate evidence, or the harms outweigh the benefits.

In formulating the recommendations for this guideline, the guideline development groups recognised and took into account several factors and limitations pertaining to the available evidence. For many aspects of PCOS, there is 
little or no evidence or the evidence is of poor quality, with other potential biases resulting from different methods for diagnosis of PCOS and differing end points.

Public and targeted consultation on the draft guideline was conducted for 30 days commencing 5 March 2011, in accordance with the legislative requirements for approval of externally developed guidelines under Section 14A of the National Health and Medical Research Council Act 1992 (Cwlth). All aspects of the guideline were developed as outlined in the NHMRC standards and procedures for externally developed guidelines, ${ }^{55}$ and accordingly, the guideline was approved by the NHMRC in July 2011. In approving the full version of the guideline, the NHMRC is satisfied that it is based on the systematic identification and synthesis of the best available scientific evidence and makes clear recommendations for health professionals practising in an Australian health care setting.

This guideline does not seek to provide full safety and usage information on pharmacological and surgical interventions. The pharmacological and surgical interventions recommended in the guideline should not be applied without consideration of the patient's clinical profile and personal preferences. It is recommended that the reader consults the Therapeutic Guidelines (http:// www.tg.com.au) and the National Prescribing Service (http://www.nps.org.au) for detailed prescribing information, including indications, drug dosages, methods and routes of administration, contraindications, supervision and monitoring, product characteristics, and adverse effects.

It is intended that this evidence-based guideline summary be used alongside the full guideline. ${ }^{8}$ The guideline should be considered according to the limitations outlined within, and used in conjunction with clinical judgement and patient preference. For a detailed description of the methodology used to develop the guideline, please see the full guideline. ${ }^{8}$

Translation of the guideline, including the production and dissemination of guideline-associated tools and resources, is the responsibility of the Jean Hailes Foundation for Women's Health as a national not-forprofit women's health organisation funded by the federal government. The PCOS Alliance and POSAA provided significant contribution to these resource developments.

Summary of the evidence-based guideline for PCOS

The recommendations in the guideline are summarised in the algorithms and are outlined in the following sections. 


\section{Summary of the evidence-based guideline}

\section{Section 1. Challenges in diagnostic assessment of PCOS (Algorithm 1)}

PCOS is diagnosed based on at least two of: oligo- or anovulation, hyperandrogenism (clinical and/or biochemical) and polycystic ovaries on ultrasound after exclusion (where relevant) of other conditions such as thyroid dysfunction, congenital adrenal hyperplasia, hyperprolactinaemia, androgen-secreting tumours and Cushing syndrome (Figure 2). ${ }^{14}$ Diagnosis of PCOS is challenging, as the presenting symptoms and signs are heterogeneous, depending on populations studied, degree of obesity and life stage of the women affected; indeed, clinical features can vary over time within an individual woman. The key identified challenges in diagnosis prioritised in the guideline included assessment of biochemical hyperandrogenism, and diagnosis of ovulatory dysfunction, polycystic ovaries and PCOS in adolescents.

Clinical question. In women with suspected PCOS, what is the most effective measure to diagnose PCOS-related hyperandrogenism?

Recommendations

Clinical consensus recommendation

1.1a Late-onset congenital adrenal hyperplasia, although rare, needs to be considered before the diagnosis of polycystic ovary syndrome is confirmed. In more severe clinical cases of hyperandrogenism, 21-hydroxylase deficiency, the most common form of congenital adrenal hyperplasia, can be excluded by measuring serum 17hydroxyprogesterone in the follicular phase to explore this diagnosis.

Evidence-based recommendation

1.1b Calculated bioavailable testosterone, calculated free testosterone or free androgen index should be first line investigation for biochemical determination of hyperandrogenism in polycystic ovary syndrome.

The addition of androstenedione and

dehydroepiandrosterone sulfate could be second line investigation for biochemical determination of hyperandrogenism in polycystic ovary syndrome. - level C Clinical practice points

1.lc It is difficult to assess androgen status in women on the oral contraceptive pill as effects include oestrogen mediated increases in sex hormone-binding globulin and reduction in androgens.

Where the oral contraceptive pill has already been commenced, it should be withdrawn for at least three months before appropriate hormonal assessments for diagnosis of polycystic ovary syndrome are undertaken. Contraception should be otherwise managed during this time.

1.1d If androgen levels are markedly above laboratory reference ranges, secondary causes may be considered. Mild elevations of androstenedione may be seen in polycystic ovary syndrome, whereas marked elevations are more indicative of non-classical adrenal hyperplasia. Reference ranges for different methods and different laboratories vary widely and clinical decisions should be guided by the reference ranges of the laboratory used.
Context. Hyperandrogenism is a well established contributor to PCOS aetiology, detected in around 60\%$80 \%$ of cases, yet it is challenging to diagnose. It includes clinical and biochemical hyperandrogenism. When determining biochemical hyperandrogenism, accurate diagnosis is hampered by a lack of ideal methods of measurement. Testosterone assays are generally designed for use in males, with poor accuracy noted at the lower androgen levels characteristic of females. In this setting, calculated measures of free androgens are preferred for diagnosis of PCOS. To improve accuracy, testosterone should ideally be measured in the morning in the follicular phase (in cycling women). For those with irregular cycles, measurement of $\mathrm{FSH}$, luteinising hormone, oestradiol and progesterone may be helpful to assess the phase of cycle and thus interpretation of androgen levels. The optimal methodology for testosterone measurement on which to base calculated measurements is unclear; however, Davison and colleagues have described a highly sensitive direct manual radioimmunoassay in the Australian setting that more accurately measures total testosterone than other assays, and that provides reference values for androgens by age. ${ }^{58}$

Calculated measures of androgen status in PCOS include calculated bioavailable testosterone and calculated free testosterone, which can be used interchangeably with calculated free testosterone, the most commonly measured marker, using the formula of Vermeuelen and colleagues. ${ }^{59}$ The free androgen index (FAI) is also a widely used measure of androgens among women, calculated based on testosterone and sex hormone-binding globulin (SHBG) levels. Other androgens, including androstenedione and dehydroepiandrosterone sulfate (DHEAS), can also be measured. These can be mildly elevated in PCOS; however, these additional androgen tests are primarily indicated in women with rapidly progressive virilisation or more severe clinical hyperandrogenism. DHEAS elevation is more marked among patients with androgen-secreting adrenal tumours, and androstenedione levels are especially elevated among patients with non-classical congenital adrenal hyperplasia. Given the controversy, methodological challenges and variety of different options for the biochemical measurement of androgens, there is uncertainty and inconsistency in clinical practice; hence, this was prioritised during the process of guideline development.

Evidence informing the recommendations. Three diagnostic studies (NHMRC level III-2) with low, moderate and high risk of bias compared the diagnostic accuracy of different reproductive hormone markers in serum to detect PCOS. ${ }^{60-62}$ For diagnosis of PCOS, calculated bioavailable and free testosterone were better than androstenedione, SHBG and total testosterone levels, and the FAI was better than androstenedione and SHBG levels. ${ }^{61}$ The remaining two studies provided insufficient 
lower-quality evidence to support any differences between total testosterone, SHBG and androstenedione levels with respect to their value in PCOS diagnosis. ${ }^{60,62}$

Implications of the recommendations. Because different laboratories currently use different methodologies, specific cut-off values to determine hyperandrogenism cannot be recommended and should be based on appropriate laboratory-specific reference intervals. Current practice in androgen measurement in PCOS is unclear, so the recommendation may require changes in usual care. There may be resource implications, as the cost of a calculated testosterone test involves measurement of testosterone and SHBG levels, and this test is more expensive than a testosterone level alone. However, more targeted first-line androgen testing may also reduce costs. The field of androgen testing is constantly changing and is likely to keep changing in the future. New automated technology (liquid chromatography mass spectrometry) is currently being introduced in many laboratories. This will need to be considered when implementing these recommendations.

Clinical question. In adolescents, at what time point after onset of menarche do irregular cycles indicate ongoing menstrual dysfunction?

Recommendations

Clinical consensus recommendation

$1.2 \mathrm{a}$ In adolescent women (< 18 years), after two years of irregular cycles ( $>35$ or $<21$ days) following the onset of menarche, polycystic ovary syndrome should be considered and appropriate assessment should be undertaken

As polycystic ovary syndrome is a diagnosis of exclusion, other causes of irregular cycles (such as thyroid dysfunction or hyperprolactinemia) need to be considered and excluded prior to the diagnosis of polycystic ovary syndrome.

Clinical practice point

1.2b If oral contraceptive pill therapy is being considered or has commenced in adolescents ( $<18$ years), the following are recommended:

- After twelve months of irregular cycles (> 35 or < 21 days) after onset of menarche, polycystic ovary syndrome should be considered before commencement of the oral contraceptive pill.

- Where the oral contraceptive pill has already been commenced, when girls are not sexually active, if biochemical hyperandrogenism is needed for the diagnosis of polycystic ovary syndrome, the oral contraceptive pill could be withdrawn for three months to facilitate appropriate hormonal assessments. Withdrawal of the oral contraceptive pill may facilitate assessment and early diagnosis of polycystic ovary syndrome as diagnosis can have important implications including optimisation of healthy lifestyle, regular metabolic screening and proactive fertility planning, with consideration of planning for conception at an earlier age. However, the risk of unplanned pregnancy needs to be considered and weighed up against potential benefits of early diagnosis. Contraception may still need to be otherwise managed during this time.
Context. Physiologically, during the first year postmenarche, hormonal responses do not match adult patterns. During the second year, hormonal profiles are similar to control-group adult women during both phases of the menstrual cycle, although progesterone levels remain lower. ${ }^{63}$

The average adult menstrual cycle is 28 days, with a normal cycle range of 24 to 35 days. ${ }^{64}$ However, during the first few years postmenarche, cycles vary considerably. ${ }^{64-66}$ In the first postmenarcheal year, around half of cycles are anovulatory. Nonetheless, $80 \%$ of cycles occur within a predictable range of 21 to 45 days. ${ }^{65-68}$ By the third postmenarcheal year, $95 \%$ of cycles fall into this range; as such, the minority without regular cycles after 2 years postmenarche should be investigated for PCOS.

Another clinical consideration when determining whether cycle irregularity is normal or abnormal in adolescents is that the onset of regular ovulatory cycles is related to age at menarche. ${ }^{69}$ Of girls who begin menses at before 12 years of age, between 12 and 13 years, and after 13 years of age, $50 \%$ of cycles are ovulatory by 1 year, 3 years, and 4.5 years, respectively. ${ }^{69}$ Weight and exercise influence the menstrual cycle of women in many ways and should always be evaluated when assessing a women with menstrual disturbance. As it remains unclear at what stage irregular cycles in adolescents reflect immaturity of the reproductive system or possible PCOS, this question was prioritised during the process of guideline development.

Evidence informing the recommendations. We did not identify any evidence from our PCOS patient population to answer the question; therefore, a clinical consensus recommendation has been made, informed by the natural history of menstrual cycles and ovulation in adolescents (aged $<18$ years).

Implications of the recommendations. Irregular cycles ( $>35$ or $<21$ days) that continue for more than 2 years after the onset of menarche are likely to reflect oligo- or anovulation. Consideration should be given to age of menarche. Current practice is unclear. It is likely that current practice involves prescription of the oral contraceptive pill without diagnosis of PCOS in adolescents with irregular cycles. This recommendation may increase referral for diagnostic testing and specialist care; however, the benefits of early diagnosis and prevention of associated complications and infertility are likely to result in significant health and cost benefits. Focus on lifestyle management, rather than medical management, may also be increased.

Clinical question. In adolescents, what are the most effective criteria to diagnose polycystic ovaries on ultrasound?

Recommendations

Clinical consensus recommendation

1.3a Given the apparent lack of specificity of polycystic ovaries on ultrasound in adolescents, generally, ultrasound should not be recommended first line in this age group for diagnosis of polycystic ovary syndrome pending further research. If pelvic ultrasounds are to be ordered in adolescents, the results should be interpreted with caution.

Clinical practice point

1.3b Vaginal ultrasound is not appropriate in adolescents who have not been sexually active. 
Context. Between the ages of 2 and 9 years, the size and morphology of the ovaries are relatively stable, with the volume of each ovary being less than $2 \mathrm{~cm}^{3}$; however, from around 9 years of age onward, the ovaries undergo progressive increases in size. ${ }^{70}$ The number of large antral follicles and ovarian size reaches its maximum around the time of menarche ${ }^{71,72}$ and many girls with regular menstrual patterns may have polycystic ovaries. ${ }^{71}$ Recently, on community-based screening, up to $68 \%$ of $19-21$-yearold Danish women were shown to have polycystic ovaries according to the Rotterdam criteria. ${ }^{16}$ As follicle counts are high at a younger age, this needs to be considered to avoid overdiagnosis of PCOS. ${ }^{16}$ It seems unlikely that the adult criteria for diagnosing PCOS according to polycystic ovaries (presence of 12 or more follicles in each ovary measuring 2-9 $\mathrm{mm}$ in diameter, and/or increased ovarian volume $[>10 \mathrm{~mL}]^{14}$ ) are directly applicable to adolescents. There is continuing discussion as to the quantitative characteristics of a polycystic ovary given increasing resolution of ultrasound. The appropriateness of vaginal ultrasound in adolescents who may not yet have been sexually active has also been questioned. Given the controversy in this area, it was prioritised during the process of guideline development.

Evidence informing the recommendations. One level III3 case-control study with moderate risk of bias ${ }^{73}$ was identified by our search to answer the question, and was deemed insufficient evidence on which to base a recommendation. Therefore, a clinical consensus recommendation was made, based on the clinical expertise of the multidisciplinary guideline development group, informed by observational data in this patient population.

Implications of the recommendations. There is a high prevalence of polycystic ovaries among adolescents, and normal reference ranges for this age group have not been developed or validated. Hence, pelvic ultrasound should be used and interpreted with caution in this age group. Original NIH criteria based on ovulatory disturbance and hyperandrogenism may be the optimal way to diagnose PCOS in adolescents. Although current practice is unclear, it is anticipated that the recommendations may result in less use of ultrasound in PCOS diagnosis in adolescents.

Research recommendation

Further methodologically rigorous trials are important to determine:

1 Natural history of menstrual cycles in PCOS compared to non-PCOS adolescents

2 Age-appropriate normal ranges for sonographic features of polycystic ovaries and for clinical and biochemical features of PCOS in adolescents (< 18 years)

3 Accuracy of ovarian volume to diagnose polycystic ovary syndrome in adolescents (<18 years). 


\section{Section 2. Interdisciplinary model of care in diagnosed PCOS (Algorithm 2)}

Clinical question. What is the effectiveness of an interdisciplinary model of care compared to usual care in terms of biopsychosocial wellbeing?

Recommendations

Clinical consensus recommendation

2.la Interdisciplinary care, with multiple health

professionals involved, should be offered to women with polycystic ovary syndrome, where appropriate based on the chronic and complex nature of the disease.

An interdisciplinary care model is the collaboration between a woman with polycystic ovary syndrome and a care team who have shared goals for her total wellbeing. It should have the following integral components:

- A care team, comprised of representation from varied health disciplines (eg, may include dietetics, psychology, endocrinology, gynaecology, exercise physiology, general practice)

- A care plan which has been developed and agreed with the woman, and if relevant, the carer

- A designated care coordinator, who oversees the care plan and monitors and evaluates outcomes, which is often the general practitioner

- Clear and regular communication (eg, information sharing via different forms of media, including internet, letters, case conferencing, email, teleconference)

The complexity of the woman's need will determine the extent of interdisciplinary care required.

Clinical practice point

2.1b When referring a woman with polycystic ovary syndrome to other health professionals (ie, psychologists), a resource has been developed (Appendix IV in the full guideline ${ }^{8}$ ) to inform the professional about polycystic ovary syndrome.

Figure 4. Patient-centred interdisciplinary model of care incorporating one or more health professionals with emphasis on shared decision making, mutual respect between all collaborating members, interactive communication and active patient empowerment

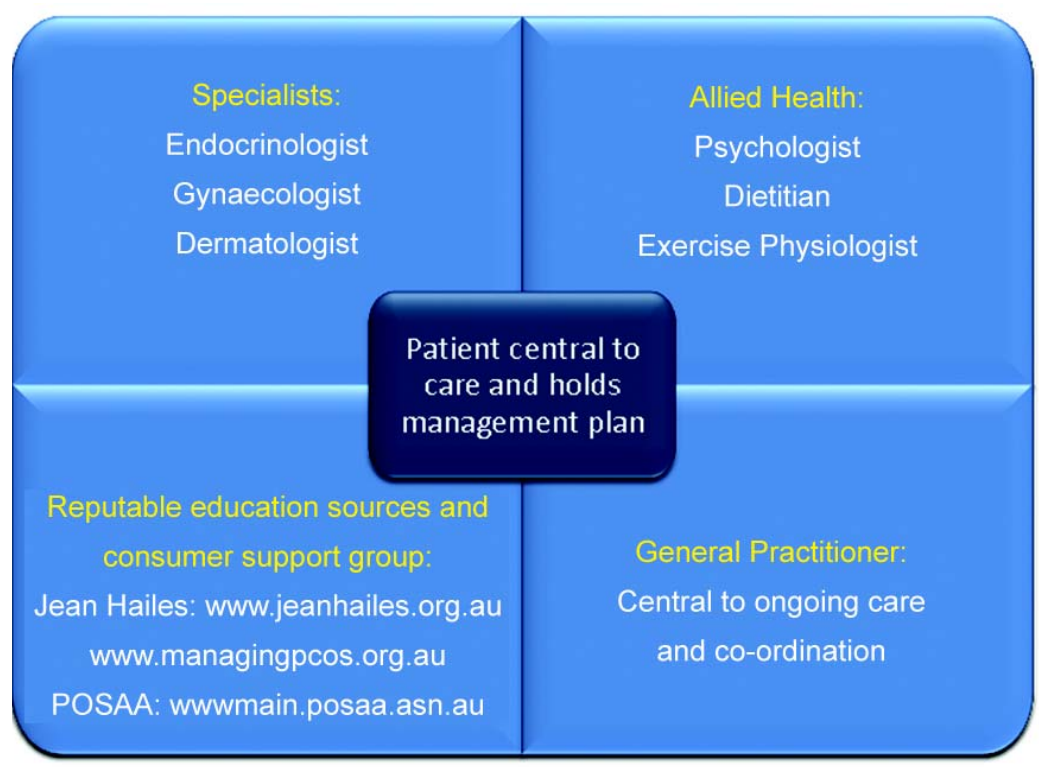

Context. Once diagnosed, a woman with PCOS may benefit from care from a variety of health professionals to manage the metabolic, reproductive and psychological features of PCOS across her life span. In addition to a GP, women with PCOS may need to engage with a dietitian, psychologist, exercise physiologist, endocrinologist, gynaecologist and infertility specialist. It appears that most women with PCOS do not adequately access these disciplines, and when they do it is usually via a GP referral to separate multidisciplinary health professionals, with communication between health professionals and limited engagement and carriage of the care plan by the patient with PCOS.

Multidisciplinary team approaches have been used in the management of several chronic diseases. A multidisciplinary approach has been credited with improvements in health-related behaviours, health parameters and general wellbeing. However, limitations of the multidisciplinary care model include compartmentalisation of patient care and limited communication between health professionals. These considerations, and the drive to more patient-centred care, have led to recommendations for an interdisciplinary model of care in chronic conditions. Interdisciplinary care involves collaboration between health professionals with clear communication pathways, and partnering with patients to address health issues with common care goals and active patient empowerment. Elements of a successful collaboration include "a willingness to collaborate, trust in each other, mutual respect and communication". ${ }^{74}$ Successful collaboration also relies on adequate systems and organisational supports. ${ }^{75}$ Although seemingly intuitive, a recent Cochrane review revealed only poorquality studies, ${ }^{76}$ highlighting a key research gap and the need for evaluation strategies in development and implementation of interdisciplinary models of care. For these reasons, this area was prioritised during the process of guideline development.

Evidence informing the recommendations. We did not identify PCOS-specific evidence to answer the question, so a clinical consensus recommendation was made based on clinical expertise, informed by the literature in other chronic disease areas.

Implications of the recommendations. Current PCOS management involves one or more health professionals managing individual components of the syndrome (Figure 4). As interdisciplinary care emphasises shared decision making, mutual respect between all collaborating members, interactive communication and active patient empowerment, changes to current management and change in the cultural mindset and philosophy of clinicians may be required. Current Australian funding arrangements may hinder community-based interdisciplinary care. Medicare funding for allied health professionals is capped, and although care plans facilitate chronic PCOS care, these may be underutilised and may benefit from adaptation for complex chronic health problems like PCOS. 
Research recommendation

Further methodologically rigorous trials are important to address implementation and evaluation of interdisciplinary models of care in polycystic ovary syndrome, where an evaluation strategy for a service model needs to be designed from the outset of the interdisciplinary service. Implementation research/evaluation of health service models requires resources. 


\section{Section 3. Assessment of cardiometabolic risk in PCOS (Algorithm 1)}

Clinical question. In women with PCOS, what is the most effective tool/method to assess risk of cardiovascular disease?

Recommendations

Clinical consensus recommendation - cardiovascular risk

3.1a All women with polycystic ovary syndrome should be assessed for cardiovascular disease risk by assessing individual cardiovascular disease risk factors.

If screening in women with polycystic ovary syndrome shows that any of the following cardiovascular disease risk factors are present, these women should be considered at increased relative risk of cardiovascular disease (obesity, cigarette smoking, dyslipidemia, hypertension, impaired glucose tolerance, lack of physical activity) and those with metabolic syndrome and/or type 2 diabetes, at even greater risk.

Clinical consensus recommendation - weight and cigarette smoking

3.1b All women with polycystic ovary syndrome should be assessed for excess weight at every visit.

In assessing women with polycystic ovary syndrome $<18$ years, age appropriate and gender appropriate body mass index should be calculated at every visit.

All women with polycystic ovary syndrome should be assessed for cigarette smoking.

Clinical practice point

3.1c Body mass index should be assessed in all women with polycystic ovary syndrome using the following criteria: ${ }^{54}$

" Body mass index $<18.5 \mathrm{~kg} / \mathrm{m}^{2}=$ underweight

- Body mass index $18.5-24.9 \mathrm{~kg} / \mathrm{m}^{2}$ = lean

" Body mass index $25.0-29.9 \mathrm{~kg} / \mathrm{m}^{2}=$ overweight

" Body mass index $30.0-34.9 \mathrm{~kg} / \mathrm{m}^{2}=$ obese

- Body mass index $\geqslant 35 \mathrm{~kg} / \mathrm{m}^{2}=$ morbidly obese

Significant benefits have been demonstrated with 5-10\% weight loss in overweight women with polycystic ovary syndrome and is a feasible initial target (see 5.4C).

Body mass index doesn't always reflect adverse body fat stores and waist circumference will be useful.

Waist circumference should be assessed using the following criteria: ${ }^{54}$

- Waist circumference $>80 \mathrm{~cm}=$ increased risk of metabolic complications

" Waist circumference $>88 \mathrm{~cm}=$ substantially increased risk of metabolic complications

Clinical consensus recommendation - lipid profile 3.1d A complete lipid profile should be measured every two years in women with polycystic ovary syndrome who have normal lipid profiles.

A complete lipid profile should be measured annually in women with polycystic ovary syndrome who have abnormal lipid profiles and/or excess weight.

Clinical practice point

3.1e In women with polycystic ovary syndrome, a lipid profile should include:

- Total cholesterol - total cholesterol should be

$<4 \mathrm{mmol} / \mathrm{L}^{77}$

- Low-density lipoprotein cholesterol (LDL-C) - in women without additional cardiovascular disease risk factors, LDL-C levels should be $<3.4 \mathrm{mmol} / \mathrm{L}^{.7}{ }^{7}$ In women with metabolic syndrome or type 2 diabetes, LDL-C levels should be $<1.8-2.6 \mathrm{mmol} / \mathrm{L}$ or $1.8 \mathrm{mmol} / \mathrm{L}$, respectively ${ }^{78}$
- High-density lipoprotein cholesterol (HDL-C) - HDL-C levels should be $>1.0 \mathrm{mmol} / \mathrm{L}^{79}$

- Triglycerides - triglyceride levels should be $<1.7 \mathrm{mmol} / \mathrm{L}^{78}$

Clinical consensus recommendation - blood pressure

3.1f Blood pressure should be measured annually in women with polycystic ovary syndrome and a body mass index $<25 \mathrm{~kg} / \mathrm{m}^{2}$ (lean).

Blood pressure should be routinely measured at each visit in women with polycystic ovary syndrome and a body mass index $\geqslant 25 \mathrm{~kg} / \mathrm{m}^{2}$ (overweight/obese).

Clinical practice point

$3.1 \mathrm{In}$ women with polycystic ovary syndrome who are at high risk of type 2 diabetes, the ideal daytime blood pressure should not exceed $135 \mathrm{mmHg}$ systolic and $85 \mathrm{mmHg}$ diastolic. ${ }^{80}$

Clinical consensus recommendation - type 2 diabetes 3.1h Prediabetes and/or type 2 diabetes should be assessed in all women with polycystic ovary syndrome (see 3.2a and 3.2b).

Context. Women with PCOS often have increased cardiovascular risk factors (hyperinsulinaemia, dyslipidaemia, hypertension) and increased prevalence of metabolic syndrome $^{81}$ (Figure 5). Dyslipidaemia in PCOS is underpinned by overproduction of triglyceride-rich lipoproteins and increased catabolism of high-density lipoproteins ${ }^{82}$ in the setting of IR and excess visceral and hepatic fat. ${ }^{82}$ In PCOS, other novel CVD risk factors, premature cardiovascular dysfunction and subclinical CVD ${ }^{29,30,83}$ have been noted and are related to IR and obesity. ${ }^{84-89}$ This evidence, along with high diabetes risk, suggests an increased relative risk of CVD in PCOS.

Two large epidemiological studies have reported increased prevalence of CVD among women with anovulation; $; 0,91$ however, poor-quality long-term PCOS studies have not shown increased CVD. ${ }^{89,92}$ In contrast, Shaw and colleagues examined a subset of postmen-pausal women from the Women's Ischemia Syndrome Evaluation study who had displayed clinical features consistent with PCOS and confirmed increased cardiovascular events and deaths. ${ }^{22}$

Figure 5. Cardiometabolic risk factor assessment in women with polycystic ovary syndrome (PCOS)

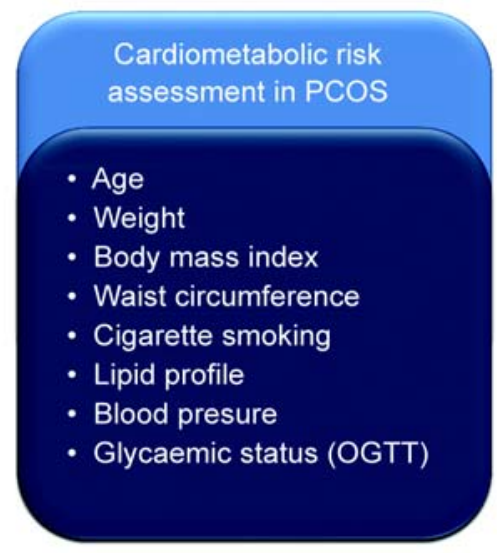

OGTT = oral glucose tolerance test 
CVD is the biggest killer of Australian women and approximately $80 \%$ is preventable through lifestyle change. The public health impact of early identification and targeted prevention in high-risk, young, reproductiveage women with PCOS is likely to be very significant; ${ }^{93-96}$ however, the best method for assessing cardiovascular risk in women with PCOS remains unclear and was prioritised during the process of guideline development.

Evidence informing the recommendations. We did not identify high-quality PCOS-specific evidence to answer the question, hence a clinical consensus recommendation was made based on clinical expertise, an international position statement on CVD risk assessment in $\mathrm{PCOS}^{78}$ and on national guidelines on absolute CVD risk assessment, ${ }^{46}$ management of obesity, ${ }^{54}$ lipids $^{77,79}$ and hypertension ${ }^{80}$ for the general population.

Implications of the recommendations. PCOS management often focuses on reproductive features and CVD risk is often not considered. A shift in cultural mindset to acknowledge the cardiovascular features of PCOS may be required. The recommendation to screen for CVD risk factors may have resource implications, with more frequent investigations; however, early detection may motivate lifestyle changes. These recommendations also highlight the need for a stronger focus on reducing excess weight and prevention of weight gain in PCOS. Also, the adverse impact of weight gain needs to be highlighted here, especially in high cardiovascular risk populations, where consideration should be given to potentially lower BMI limits.

Clinical question. In women with PCOS, what is the most effective tool/method to assess risk of type 2 diabetes?

Recommendations

Clinical consensus recommendations

3.2a To assess for risk of type 2 diabetes, in addition to polycystic ovary syndrome status, the following diabetes risk factors should be considered:11,97

" Age

- Gender

" Ethnicity

- Parental history of diabetes

- History of high blood glucose level

" Use of antihypertensive medications

- Smoking

- Physical inactivity

" Waist circumference

3.2b An oral glucose tolerance test should be performed every second year in all women with polycystic ovary syndrome and annually in those found to have additional risk factors for developing type 2 diabetes as outlined in 3.2a. Clinical practice point

3.2c Reference ranges for: ${ }^{98}$

- Impaired fasting glucose - fasting plasma glucose: $6.1-6.9 \mathrm{mmol} / \mathrm{L}$

- Impaired glucose tolerance - 2-hour glucose level: $7.8-11 \mathrm{mmol} / \mathrm{L}$

- Type 2 diabetes - fasting plasma glucose: $\geqslant 7.0 \mathrm{mmol} / \mathrm{L}$; or 2-hour oral glucose tolerance test: $\geqslant 11.1 \mathrm{mmol} / \mathrm{L}$.

Ideally, 150 grams of carbohydrate per day should be consumed for 3 days before, and women should then fast for 8 hours immediately before the oral glucose tolerance test, since low carbohydrate intake may lead to false positive glucose tolerance tests.
Context. IR is a key aetiological feature in PCOS, worsening the reproductive and metabolic features, type 2 diabetes and CVD risk. ${ }^{30,99,100} \mathrm{IR}$ is present in around 50\%$80 \%$ of women with PCOS, independent of obesity, ${ }^{101}$ and is further exacerbated by excess weight. ${ }^{20,21,102}$ Women with PCOS have increased Finnish Diabetes Risk Scores as well as increased risk of gestational diabetes, ${ }^{24}$ prediabetes and $\mathrm{DM} 2^{11,103}$ — independent of, but exacerbated by, obesity. Earlier-onset hyperglycaemia and rapid progression to DM2 is also reported in PCOS. ${ }^{104,105}$ In this context, PCOS is listed by the International Diabetes Federation as a non-modifiable risk factor for DM2. ${ }^{106}$ Furthermore, lifestyle therapy has been shown to prevent or delay progression to DM2.

Previous studies have noted that fasting blood glucose level alone appears to lack sensitivity in screening for prediabetes or DM2 in PCOS, with national and international groups recommending screening all women with PCOS with an OGTT. ${ }^{78,107}$ It is important to note that assessment of IR or measurement of serum insulin levels has no current role in clinically assessing for risk of DM2. Although the high risk of DM2 is recognised in PCOS and screening is important to facilitate effective lifestyle therapy to prevent DM2, which patients to target, how to test and at what frequency remain unclear and were prioritised during the process of guideline development.

Evidence informing the recommendations. We identified one low-quality systematic review (level I) with high risk of bias; ${ }^{108}$ however, this was deemed insufficient evidence on which to base the recommendation. A clinical consensus recommendation was made based on the previous national and international publications suggesting screening all women with PCOS with an OGTT, ${ }^{78,107}$ on the systematic review, on a recent an international position statement on CVD risk assessment in $\mathrm{PCOS}^{78}$ and on national guidelines for case detection and diagnosis of DM2. ${ }^{98}$

Implications of the recommendations. The significantly increased risk of DM2 in PCOS represents a major health and economic burden. The recommendation to screen all women with PCOS and to use an OGTT will result in

Figure 6. Anxiety and depression, poor body image, eating disorders and psychosexual dysfunction are all likely to affect quality of life in polycystic ovary syndrome

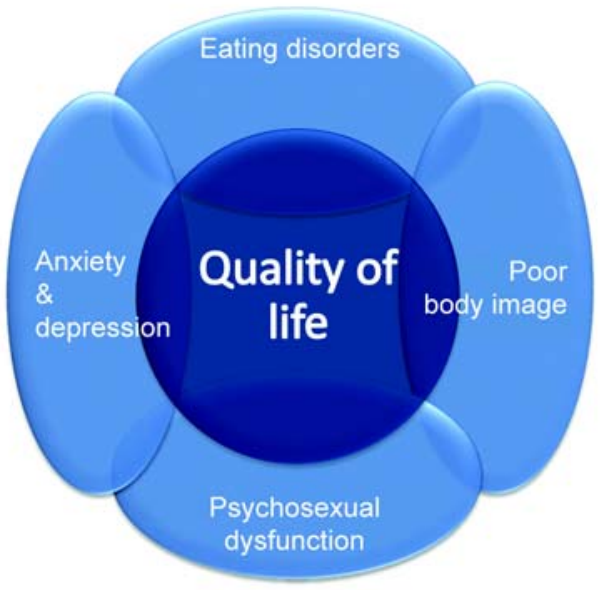


earlier detection of prediabetes and DM2, and will present opportunities for prevention of DM2 and its complications, through effective lifestyle intervention, as outlined below. Resource implications include increased screening tests, counterbalanced by earlier detection and potential for prevention. However, it is acknowledged that OGTTs increase inconvenience for patients, which may affect compliance. Other anticipated changes to diagnosis of diabetes may impact on these recommendations including the role of glycated haemoglobin, which is currently under review at national and international levels.
Research recommendation

Further methodologically rigorous trials in women with polycystic ovary syndrome are important to determine:

1 Absolute risk of CVD in women with polycystic ovary syndrome across age ranges

2 The most appropriate way of identifying those with polycystic ovary syndrome at highest risk of developing type 2 diabetes and the value of utilising existing scores such as the AUSDiab risk score in women with polycystic ovary syndrome

3 The most effective method to prevent the development of type 2 diabetes

4 The risk of diabetes and cardiometabolic issues in relatives of women with PCOS. 


\section{Section 4. Assessment of emotional wellbeing in PCOS (Algorithm 2)}

Clinical question. In women with PCOS, what is the most effective tool/method to assess depression and/or anxiety? Recommendations

\section{Clinical consensus recommendation}

4.1a Depression and/or anxiety should be routinely screened and assessed by all appropriately qualified health professionals in women with polycystic ovary syndrome.

If a woman with polycystic ovary syndrome is positive on screening, the practitioner should further assess for depression and/or anxiety.

If depression and/or anxiety are detected, appropriate management should be offered.

Clinical practice point

4.1b To screen for depression and/or anxiety, the following questions could be asked:41

1 During the last month, have you often been bothered by feeling down, depressed, or hopeless?

2 During the last month, have you often been bothered by having little interest or pleasure in doing things?

3 During the last month, have you been bothered by feeling excessively worried or concerned?

If any of the screening questions are positive further depression and/or anxiety assessment could be by either:

a Referring the patient to an appropriate professional if they do not feel competent to perform a further mental health assessment. If the health professional is not the patient's usual GP, inform the GP of the referral.

$b$ If they feel competent, perform a clinical interview and according to level of competence, choose from one or more of the following:

- Kessler Psychological Distress Scale 10 (K10)

- Depression Anxiety Stress Scale (DASS21)

- Patient Health Questionnaire (PHQ9)

- Generalized Anxiety Disorder 7-item scale (GAD7).

Context. Prevalence of depression among women with PCOS is higher $(28 \%-64 \%)^{5,109,110}$ than in the general population (7.1\%-8.0\%). ${ }^{111,112}$ Prevalence of anxiety among women with PCOS ranges from $34 \%{ }^{113}$ to $57 \%{ }^{5}$ again, higher than in the general population $(18 \%) .{ }^{111}$ Women with PCOS are also likely to experience more severe anxiety and depression. ${ }^{5,10}$ Contributing factors remain controversial, but include clinical symptoms of PCOS, excess weight, and the chronic and complex nature of PCOS. The higher prevalence and severity of mood disorders in PCOS is likely to affect self-efficacy and ability to adhere to lifestyle management in PCOS. Mood also adversely affects overall QoL. Assessment of depression and/or anxiety for women with PCOS is important, as recognition and treatment improves emotional wellbeing and QoL (Figure 6) and is likely to improve effectiveness of lifestyle intervention in PCOS. Given that recognition of depression and anxiety remains poor in PCOS, screening and assessment was prioritised during the process of guideline development.

Evidence informing the recommendations. We did not identify any PCOS-specific evidence to answer the question, so a clinical consensus recommendation was made based on key relevant sources of evidence-based information for mood disorders in the general population combined with the clinical expertise of the multidisciplinary guideline development group. Several other relevant national and international guidelines were also used to inform the recommendations. ${ }^{36,38,41-43}$

Implications of the recommendations. It is the responsibility of all health professionals to understand the impact of PCOS on psychological health and to screen, assess and manage these disorders. However, it is not usual practice to screen women with PCOS for depression and/or anxiety. Screening may uncover undiagnosed mood disorders with resource implications including increasing consultation duration. This can be attenuated by the use of the freely available recommended tools and the guidelinedeveloped brief PCOS emotional wellbeing general screening tool (see Appendix $\mathrm{V}$ in the full guideline). ${ }^{8}$ If depression and or anxiety are detected, intervention may require referral to other health practitioners or the use of other avenues (eg, an Australian GP Mental Health Care Plan), to optimise access to relevant mental health professionals. Access to appropriately trained health professionals will be required.

Clinical question. In women with PCOS, what is the most effective tool/method to assess body image?

Recommendations

Clinical consensus recommendation

4.2a Negative body image should be considered in women with polycystic ovary syndrome.

If a woman with polycystic ovary syndrome is positive on screening, the practitioner should further assess for negative body image.

If negative body image is detected, appropriate management should be offered.

Clinical practice point

4.2b To screen for negative body image, the following questions could be asked:

1 Do you worry a lot about the way you look and wish you could think about it less?

2 On a typical day, do you spend more than 1 hour per day worrying about your appearance? (More than 1 hour a day is considered excessive)

3 What specific concerns do you have about your appearance?

4 What effect does it have on your life?

5 Does it make it hard to do your work or be with your friends and family?

If an issue is identified, the practitioner could further assess negative body image by:

a Identifying any focus of concern of the patient and respond appropriately

b Assessing the level of depression and/or anxiety (if they have not done so already [see 4.1a])

$c$ Identifying if there is any distortion of body image (eg, presence of anorexia nervosa [see 4.3] or body dysmorphic disorder).

Context. Body image is complex and is influenced by many factors. In this guideline, body image is defined as the way a person feels about, thinks of and views their body, including their appearance. Body image includes attitudes to physical appearance, understanding of health, physical fitness and body size, the mental picture that individuals form of their body, values and self-esteem (Figure 7). 
Figure 7. Continuum of disordered eating and body image

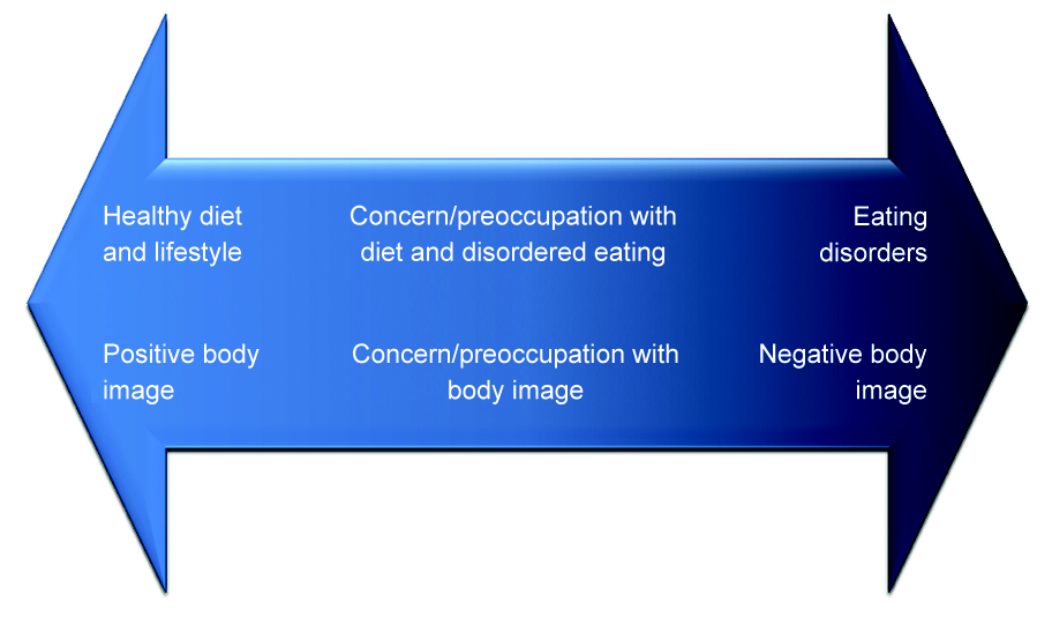

Physical factors affecting appearance (excess weight and hirsutism), psychological (self-esteem) and sociocultural factors can influence body image. Body image is generally poor in women, yet negative body image is more prevalent among women with PCOS and affects thoughts and feelings about health and appearance, QoL, mood and physical fitness. ${ }^{114,115}$ Negative body image also predicts depression and anxiety. ${ }^{116,117}$ Given the impact of negative body image on mood and QoL, body image in women with PCOS should be considered. If identified, addressing negative body image and associated mood disorders is important for improving emotional wellbeing and QoL in PCOS (Figure 6). However, body image is often not considered in PCOS; hence, this area was prioritised during the process of guideline development.

Evidence informing the recommendations. We did not identify any PCOS-specific evidence to answer the question, so a clinical consensus recommendation has been made based on key relevant sources of evidencebased information for the general population and the clinical expertise of the multidisciplinary guideline development group. ${ }^{37,39}$

Implications of the recommendations. It is not usual practice to screen women with PCOS for negative body image. However, its detection provides an opportunity to address psychological aspects, such as self-esteem and self-acceptance, and the physical aspects, such as hirsutism and excess weight. Screening may increase consultation lengths; however, this can be reduced by the emotional wellbeing screening tool and other tools recommended here (see Appendices V and VI in the full guideline) ${ }^{8}$ Intervention may require referral to other health practitioners. Where needed, access to appropriately trained and experienced health professionals is required. A shift in cultural mindset may be required to ensure the clinical impact of negative body image is understood and addressed.

Clinical question. In women with PCOS, what is the most effective tool/method to assess disordered eating? Recommendations

Clinical consensus recommendation

4.3a Disordered eating, including eating disorders, should be considered in women with polycystic ovary syndrome.
If a woman with polycystic ovary syndrome is positive on screening, the practitioner should further assess for disordered eating and eating disorders.

If disordered eating or an eating disorder is detected, appropriate management should be offered.

Clinical practice point

4.3b To screen for disordered eating and eating disorders, the following questions could be asked:

1 Do you worry you have lost control over your eating?

2 Do you ever feel disgusted, depressed, or guilty about eating?

3 Have you tried fasting or skipping meals in an attempt to lose weight?

4 Have you tried vomiting, laxatives or diuretics in an attempt to lose weight?

5 Have you had significant (eg, > 5\%-7\%), recurrent fluctuation in body weight?

If a woman with polycystic ovary syndrome answers yes to any of the above questions the practitioner should further assess for the diagnosis of an eating disorder by either:

a Referring the patient to an appropriate professional to perform a further mental health assessment.

b If the practitioner feels competent, performing a clinical interview which may be supplemented with standardised assessment tools (eg, Eating Assessment Tool [EAT-26] http://www.eat-26.com).

c Assessing the level of depression and/or anxiety (see 4.7a).

Context. Australian research suggests that prevalence of diagnosable eating disorders is increasing. ${ }^{118}$ Disordered eating refers to eating- and weight-related symptoms and can include behavioural (eg, bingeing, excessive restriction), cognitive (eg, excessive dietary restraint, negative body image) and emotional factors (Figure 7). The consequences of eating disorders encompass medical, psychological, social and occupational difficulties ${ }^{118,119}$ and are associated with an increased prevalence of mood disorders. $^{119}$

Prevalence data on eating disorders in PCOS is limited, with links between PCOS and bulimia nervosa ${ }^{120,121}$ and abnormal eating behaviour. ${ }^{120,122,123}$ The prevalence of any eating disorder has been reported at $21 \%$ in PCOS, ${ }^{124}$ with high rates of associated mood disorders; $62 \%$ had major depression and $41 \%$ anxiety disorders. Women with PCOS have higher prevalence of risk factors for eating disorders, including excess weight, depression, anxiety, low selfesteem and poor body image. ${ }^{119}$ There may also be more motivation for weight loss and prescribed and selfimposed dietary restriction in PCOS.

Available research suggests that fewer than half of patients with clinically significant eating disorders are identified in primary care, potentially related to patient ambivalence, secrecy and shame or health practitioner knowledge, attitudes and skills. Health practitioners are well placed to identify these problems, with management potentially improving physical and psychological functioning and QoL in PCOS (Figure 6); hence, this area was prioritised during the process of guideline development.

Evidence informing the recommendations. We did not identify any evidence in women with PCOS to answer the question; therefore, a clinical consensus recommendation was made based on key relevant sources of evidence-based information for the general population and the clinical 
expertise of the multidisciplinary guideline development group.

Implications of the recommendations. It is not usual practice to screen women with PCOS for disordered eating. It is likely that improved screening practices will increase identification of disordered eating/eating disorders, whereby effective treatment is available. ${ }^{125-128}$ Resource implications include potentially increased consultation lengths, which the emotional wellbeing general screening tool and other tools recommended here may assist with (see Appendices V and VI in the full guideline). ${ }^{8}$ Increased detection of disordered eating will result in increased referrals to other health professionals, and access to appropriately trained and experienced health professionals will be required. A shift in cultural mindset may be required to ensure the clinical impact of eating disorders is understood and addressed.

Clinical question. In women with PCOS, what is the most effective tool/method to assess psychosexual dysfunction?

Recommendations

Clinical consensus recommendation

4.4a Psychosexual dysfunction should be considered in women with polycystic ovary syndrome.

If a woman with polycystic ovary syndrome is positive on screening, the practitioner should further assess for psychosexual dysfunction.

If psychosexual dysfunction is detected, appropriate management should be offered.

Clinical practice point

4.4b To screen for psychosexual dysfunction, the following questions could be asked:

1 During the last few months, have you often been bothered by problems with your sex life such as reduced satisfaction, diminished desire, pain, or any other problems?

2 Do you feel that polycystic ovary syndrome affects your sex life?

3 (If relevant) Do sexual problems affect your current relationship and/or have sexual problems affected your past relationships?

If a woman with polycystic ovary syndrome answers yes to any of the above questions or where sexual function is a concern, the practitioner should assess this through more detailed clinical interview, and in particular screen for depression and/or anxiety if not already done (see 4.1) and negative body image (see 4.2) or refer to a more appropriately qualified health practitioner.

Specific validated scales could be used as outcome measures at baseline to monitor progress over time. The choice of scale should be selected by the discretion of the clinician, based on the specific sexual problem, accessibility and expertise of the practitioner.
Context. Psychosexual dysfunction refers to sexual problems or difficulties that have a psychological origin based in cognitions and/or emotions such as depression, low self-esteem and negative body image. ${ }^{129}$ It appears that women with PCOS suffer from greater psychosexual dysfunction than women in the general population with correlations between PCOS and reduced QoL, sexual satisfaction and feminine identity. ${ }^{130-132}$

Physical PCOS symptoms such as hirsutism, obesity, menstrual irregularity and infertility may cause loss of feminine identity that can affect sexuality. ${ }^{130,132,133}$ Women with PCOS also report less sexual satisfaction and lower sexual self-worth than women without PCOS, which adversely affects their relationships. ${ }^{134}$ Clinicians should be aware of potential psychosexual dysfunction in PCOS and screening and assessment should be considered.

Evidence informing the recommendations. We did not identify any PCOS-specific evidence to answer the question; therefore, a clinical consensus recommendation was made based on key relevant sources of evidence-based information for the general population and the clinical expertise of the multidisciplinary guideline development group.

Implications of the recommendations. Screening and assessment for psychosexual dysfunction should be considered in sexually active women in order to facilitate appropriate intervention aiming to optimise sexual function, limit the social impact of PCOS and improve QoL (Figure 6). It is not usual practice to screen and assess women with PCOS for psychosexual dysfunction and screening may detect undiagnosed cases. Screening may have resource implications such as an impact on length of consultation, and intervention may require referral to other specialist health professionals.

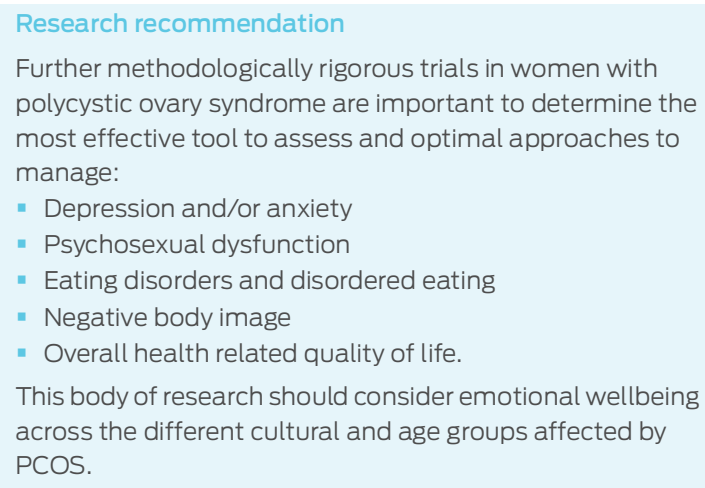

To find a psychologist, visit the Australian Psychological Society website: http://www.psychology.org.au/ FindaPsychologist. 


\section{Section 5. Lifestyle management in PCOS (Algorithm 3)}

\begin{abstract}
Clinical question. In women with PCOS, are lifestyle interventions (combined compared to minimal or nothing) effective for improving weight loss, metabolic, reproductive, fertility, quality of life and emotional wellbeing outcomes?

Recommendation

Evidence-based recommendation

5.1a Lifestyle management (single or combined approaches of diet, exercise and/or behavioural interventions) for weight loss, prevention of weight gain, or for general health benefits should be recommended in women with polycystic ovary syndrome. - level B
\end{abstract}

Context. Women with PCOS appear to be heavier than women without PCOS, and to gain more weight longitudinally. ${ }^{135,136}$ Excess weight increases the risk of developing PCOS and increases the clinical severity of the condition by exacerbating both androgen and insulin levels. ${ }^{93,102}$ Indeed, excess weight increases the severity of reproductive, ${ }^{20,21}$ psychological and metabolic features in PCOS. ${ }^{84,85}$ Along with the recognised challenges of managing established excess weight, there is a clear argument for prevention of weight gain.

Weight loss also appears to offer considerable benefits to most women with PCOS who are already overweight. There is a large number of small, uncontrolled trials demonstrating that weight loss achieved through lifestyle management decreases abdominal fat, hyperandrogenism and IR and improves lipid profiles, ovulation, menstrual cyclicity, fertility, risk factors for DM2 and CVD and psychological health in women with PCOS who are overweight. ${ }^{137-140}$ Furthermore, among women with PCOS and excess weight, a reduction of as little as $5 \%$ of total body weight has been shown to improve outcomes. $^{137-159}$

Hence, lifestyle management is useful for targeting weight loss and prevention of weight gain, and is first-line treatment for a large proportion of women with PCOS. Lifestyle management may also improve PCOS independent of weight loss, with exercise intervention improving metabolic risk factors associated with PCOS, including hypertension, IR and elevated blood glucose levels, even when no weight loss occurs. ${ }^{160-162}$ However, it is difficult to be certain about the effectiveness of lifestyle interventions in women with PCOS, because available information is based on small uncontrolled trials that address different outcomes in different subgroups of women, and specific recommendations remain unclear. Hence, this area was prioritised during guideline development.

Evidence informing the recommendations. One highquality systematic review (level I) with a low risk of bias appraised six randomised controlled trials (RCTs) (low to moderate quality and moderate to high risk of bias) and found that lifestyle intervention (diet, exercise and/or behaviour) outperformed minimal treatment for total testosterone $(P=0.004)$, hirsutism by Ferriman-Gallwey score $(P=0.04)$, weight $(P<0.001)$, waist circumference $(P=0.006)$, waist-hip ratio $(P=0.02)$, fasting insulin $(P=$
0.002) and OGTT insulin $(P<0.001)$, and percent weight change $(P<0.001)$. There was no difference between the two interventions for BMI, FAI, SHBG, blood glucose levels or lipid levels. QoL, patient satisfaction and acne were not reported. ${ }^{163}$ Due to the inconsistencies and methodological weaknesses of included studies, caution is recommended when interpreting the combined metaanalyses and results of the systematic review. None of the studies addressed fertility outcomes.

Implications of the recommendations. It is likely that the recommendation will reduce variation in practice and ensure lifestyle advice is prescribed to all women with PCOS, targeting prevention of weight gain and promoting weight loss, where appropriate. The recommendation may increase consultation times, use of care plans, and referral to allied health professionals. This may increase health care costs, but long-term benefits of lifestyle change should reduce the health and economic burden of PCOS. Barriers to implementation include engagement of health practitioners, access to allied health professionals and financial constraints. Insufficient consultation time allocated by health professionals may also be a barrier, and a shift in cultural mindset may be required to focus on lifestyle interventions, especially around prevention of weight gain. In high-risk populations, where cardiometabolic and DM2 risk is increased, the impact of weight gain appears to be more significant than in Caucasian populations, and this needs to be considered when assessing and managing PCOS.

Research recommendation

Further methodologically rigorous trials in women with polycystic ovary syndrome are important to address:

" The extent of the benefits of lifestyle management compared to no or minimal therapy for all clinically relevant outcomes

- Comparing efficacy of different types of lifestyle management (diet alone, exercise alone, behavioural modification alone, or combinations of the three)

- The effect of lifestyle management in prevention of weight gain/weight maintenance compared to weight loss

" The effect of lifestyle management for women who are both overweight and not overweight and specific reproductive outcomes such as menstrual regularity, ovulation and fertility and the relative efficacy of lifestyle management either compared to or in combination with pharmacological therapy.

Clinical question. In women with PCOS, what is the effectiveness of diet compared to exercise for improving weight loss, metabolic, reproductive, fertility, quality of life and emotional wellbeing outcomes?

Recommendations

Evidence-based recommendation

5.2a Lifestyle management targeting weight loss (in women with a body mass index $\geqslant 25 \mathrm{~kg} / \mathrm{m}^{2}$ [overweight]) and prevention of weight gain (in women with a body mass index $<25 \mathrm{~kg} / \mathrm{m}^{2}$ [lean]) should include both reduced dietary energy (caloric) intake and exercise and should be 
first-line therapy for all women with polycystic ovary syndrome. - level C

Clinical practice point

5.2b Psychological factors should be considered and managed to optimise engagement and adherence with lifestyle interventions.

Context. Identifying key components of successful lifestyle interventions may improve clinical features of PCOS and reduce complications including DM2. Although lifestyle management including diet, exercise and weight loss are first-line treatment for PCOS and should precede and accompany pharmacological treatment, there are no specific dietary or exercise recommendations in PCOS, with few comparative effectiveness studies. Lifestyle change in adults at risk of DM2 shows that targeting both exercise and diet is most successful, yet lifestyle interventions have low engagement and adherence rates, and it is clear that more research is needed to identify key successful components. Hence, this clinical question was prioritised during the guideline development process.

Evidence informing the recommendations. One lowquality non-RCT (level III-2) with a high risk of bias compared exercise to low-caloric, high-protein diet in PCOS. ${ }^{164}$ Structured exercise training (SET) was better than diet for menses frequency $(P=0.04)$ and ovulation rate $(P=0.03)$. There was no difference between interventions for anthropometric, metabolic, non-fertility or QoL outcomes; however, subgroup analysis in ovulatory women revealed that SET was better than diet for fasting insulin $(P<0.05)$, fasting glucose-to-insulin ratio $(P<0.05)$, homeostasis model of assessment of IR $(P<0.05)$, SHBG $(P<0.05)$ and FAI $(P<0.05)$. Diet was better than SET for weight $(P<0.05)$ and BMI $(P<0.05)$. This evidence is generalisable to PCOS, particularly to women who have BMI $\geqslant 33 \mathrm{~kg} / \mathrm{m}^{2}$. ${ }^{164}$ There may be limitations to generalising the findings of this study, which was conducted in Italy, to other ethnic groups. The evidence is limited to the particular exercise (bicycling) and diet (high-protein, low-carbohydrate) interventions used in the study.

Implications of the recommendations. Successful lifestyle components, including diet and exercise, separately and in combination, have significant health benefits and should be combined. It is acknowledged that there may be changes to usual care and the way care is organised, and there are potentially greater barriers to integration of exercise in lifestyle interventions for young women. However, it is expected that the cost of lifestyle interventions will be less than the cost of pharmacological intervention.

Clinical question. In women with PCOS, are diet interventions (compared to no diet or different diets) effective for improving weight loss, metabolic, fertility, and emotional wellbeing outcomes?

Recommendations

Evidence-based recommendations

5.3a Weight loss should be targeted in all women with polycystic ovary syndrome and body mass index $\geqslant 25 \mathrm{~kg} / \mathrm{m}^{2}$ (overweight) through reducing dietary energy (caloric) intake in the setting of healthy food choices, irrespective of diet composition. - level C

5.3b Prevention of weight gain should be targeted in all women with polycystic ovary syndrome through monitored caloric intake, in the setting of healthy food choices, irrespective of diet composition. - level D Clinical practice point

5.3c Weight loss (in women with a body mass index $\geqslant 25 \mathrm{~kg} / \mathrm{m}^{2}$ [overweight]) and prevention of weight gain (in women with a body mass index $<25 \mathrm{~kg} / \mathrm{m}^{2}$ [lean]) is the joint responsibility of all health professionals, partnering with women with polycystic ovary syndrome. Where complex dietary issues arise (or obesity is present), referral to a dietitian should be considered as part of an enhanced primary care plan.

Tools such as Lifescripts (http://www.health.gov.au/ lifescripts) could be used for engagement in dietary change.

Context. An area of increasing focus in PCOS is the macronutrient composition of the dietary component of a lifestyle intervention. Specific approaches including modifying carbohydrate, protein or fat are proposed to have favourable hormonal or metabolic effects or be more sustainable. Systematic reviews in the general population report similar or less weight loss and compliance with lowfat diets, ${ }^{165,166}$ and a large RCT reported similar changes in weight for a range of reduced energy diets with different macronutrient content over 2 years. ${ }^{167}$ Caloric (energy) restriction per se, rather than changes in macronutrient composition, appears most effective for weight loss and clinical benefits, and with limited research in this area in PCOS this question was prioritised during guideline development.

Evidence informing the recommendations. Six articles reporting five RCTs of moderate ${ }^{139,145,156,168}$ to high risk of bias $^{169,170}$ were identified to answer this question. The studies compared macronutrient dietary compositions, including low-carbohydrate, low glycaemic-index, monounsaturated fatty acid-enriched, conventional healthy diet, high-protein, carbohydrate-counting, or fatcounting dietary approaches. There was no difference for most anthropometric, metabolic, fertility, non-fertility, QoL and emotional wellbeing outcomes. Regardless of diet composition, a diet aimed at reducing weight yielded benefits in PCOS. The evidence is generalisable to PCOS, particularly to women over 30 years of age and who have BMI $\geqslant 30 \mathrm{~kg} / \mathrm{m}^{2}$. However, most studies recruited Caucasian women and may not be generalisable to other ethnic groups.

Implications of the recommendations. Healthy food choices (Figure 8) in energy-reduced diets, irrespective of composition, are recommended in overweight and obese women (BMI $\geqslant 25 \mathrm{~kg} / \mathrm{m}^{2}$ ) with PCOS for weight loss, and in lean women $\left(\mathrm{BMI}<25 \mathrm{~kg} / \mathrm{m}^{2}\right)$ with PCOS for prevention of weight gain. Most practitioners do not recommend specific dietary composition, so this may not change usual care. However, consumer-targeted marketing claims benefits for specific macronutrient composition in PCOS, so greater education to ensure consumers understand the principles of evidence-based dietary approaches is important. The guideline development group is not aware of resource implications, changes to the way care is 
organised, or barriers to implementation of these recommendations.

\section{Research recommendation}

Further methodologically rigorous trials in women with polycystic ovary syndrome are important to address:

1 A range of diet compositions including low glycaemic index for both prevention of weight gain/weight maintenance and weight loss in women who are overweight and in women who are lean

2 Monitoring of safety and harms of diets and longer follow-up

3 Increasing engagement and adherence with intervention studies.

Clinical question. In women with PCOS, what is the most effective method to deliver dietary information for improving weight loss, quality of life and emotional wellbeing outcomes?

Recommendations

Evidence-based recommendation

5.4a Face to face, tailored dietary advice, including education, behavioural change techniques and ongoing support should be provided to women with polycystic ovary syndrome and a body mass index $\geqslant 25 \mathrm{~kg} / \mathrm{m}^{2}$ (overweight). Dietary modification is the joint responsibility of all health professionals, partnering with women with polycystic ovary syndrome. - level C

\section{Clinical consensus recommendation}

5.4b Behaviour-change techniques should target prevention of weight gain in all women with polycystic ovary syndrome including those with a body mass index $<25 \mathrm{~kg} / \mathrm{m}^{2}$ (lean).

Clinical practice point

5.4c Behavioural change techniques, including motivational interviewing, should be used in addition to advice/education. Simple strategies, including selfmonitoring, pedometers and time management techniques should be encouraged. Interventions could be individual, group or mixed mode, in a range of settings, delivered by a range of health professionals. Individual techniques should not be used in isolation and should be part of a coherent multidisciplinary interventional model. Key messages should be reinforced with women with polycystic ovary syndrome, including that achievable goals ( $5 \%$ to $10 \%$ loss of body weight in overweight women) yield significant clinical improvements.

Context. Few comparative studies have explored methods to deliver diet and lifestyle interventions to improve weight loss among women with PCOS. In general populations, motivational interviewing and established behaviour techniques appear more effective than traditional education and advice. ${ }^{172}$ Providing instruction, establishing self-monitoring (including self-weighing and pedometer use), time-management techniques, relapseprevention techniques, individual tailoring, engaging social support and setting goals all appear useful. Individual, group and mixed intervention, and a wide range of trained providers, including doctors, nurses, dietitians, nutritionists and exercise specialists, can deliver effective lifestyle interventions. ${ }^{173-177}$

Again, in general populations, behaviour-change techniques, increased contact time with health professionals, and greater intensity and duration of
Figure 8 . Healthy food choices ${ }^{171}$

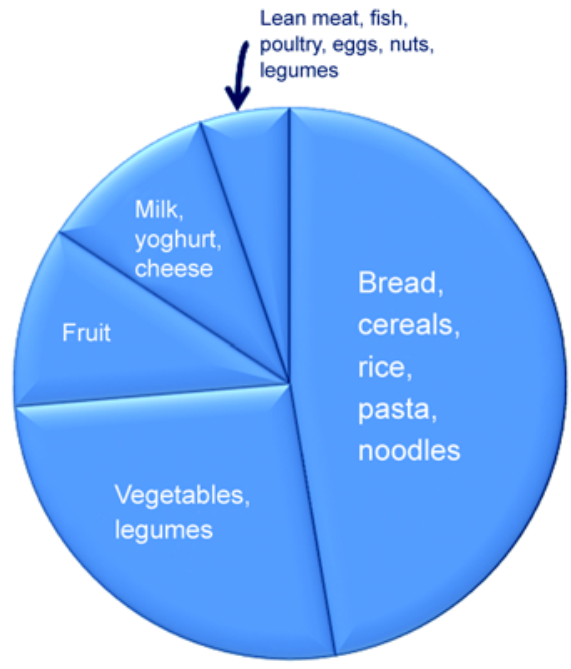

behavioural intervention all generate significantly more weight loss, ${ }^{178}$ whereas a variety of settings, including primary care and community and workplace settings, appear effective. Overall, interventions to promote changes in diet and/or exercise in adults with increased risk of DM2 or CVD are more likely to be effective if they combine intensive lifestyle interventions and sustainable behaviour-change methods. However, optimising engagement, adherence and successful outcomes in young women with PCOS remains a challenge; hence, this clinical question was prioritised during guideline development.

Evidence informing the recommendations. Eight articles reporting seven RCTs of moderate ${ }^{139,152,156,168,179}$ to high risk of bias ${ }^{169,170,180}$ were identified for this question. The multidisciplinary guideline development group included evidence comparing diet compositions as different diets have inherent differences in delivery. The included studies used various diet-delivery methods/tools (and associated support mechanisms) and macronutrient diet compositions. Studies consistently involved dietitian consultation, with monitoring varying from daily ${ }^{169}$ and weekly ${ }^{156}$ to monthly ${ }^{139,152,168,179,180}$ or mixed interval monitoring. ${ }^{152,170}$

Of the diet-delivery methods used, two studies used official dietary guidelines ${ }^{152,156,170}$ and three studies provided food for participants. ${ }^{152,169,179}$ Of these, two used partial meal replacement. ${ }^{152,179}$ Other studies changed composition of whole foods. ${ }^{139,152,156,168,170,179,180}$ Diet changes were made to all daily meals, except in the case of one study where only breakfast was altered. ${ }^{179}$ Exercise was only increased in two studies. ${ }^{139,168}$ Other studies reported regular exercise, ${ }^{152,170}$ or having no change in exercise levels. ${ }^{156,179}$ One study offered other lifestyle support mechanisms such as menu plans, emails, newsletters, articles, recipes, and motivational support. ${ }^{152,170}$

There was no difference between the diet-delivery methods for most anthropometric, QoL, and emotional wellbeing outcomes. Two studies reported differences for anthropometric outcomes, ${ }^{169,179}$ and another reported 
differences in an emotional wellbeing outcome; ${ }^{170}$ however, the studies varied by the diet-delivery regimen and by degree of support. Overall, the diet-delivery approaches that were consistently reported included faceto-face advice and education about diet composition, food types and practical approaches to healthy eating, including behaviour-change techniques.

The evidence from the included studies is directly generalisable to the patient population of PCOS, particularly to women over 30 years of age and who have BMI $\geqslant 30 \mathrm{~kg} / \mathrm{m}^{2}$. However, most of the women in the studies were Caucasian, so there may be limitations to generalising these findings to other ethnic groups.

Implications of the recommendations. Face-to-face, tailored dietary advice, including education, behavioural change techniques and ongoing support should be provided to women with PCOS for weight loss in overweight women (BMI $\geqslant 25 \mathrm{~kg} / \mathrm{m}^{2}$ ) with PCOS and for prevention of weight gain in lean women $\left(\mathrm{BMI}<25 \mathrm{~kg} / \mathrm{m}^{2}\right.$ ) with PCOS. Current practice is unclear. Dietary modification is the joint responsibility of all health professionals, partnering with women with PCOS, and these recommendations may result in increased referrals to allied health professionals. PCOS information to aid allied health management of PCOS has been developed (See Appendix IV in full guideline). ${ }^{8}$ There may be resource implications due to increased consultation times and increased use of care plans. Engagement of health practitioners and financial barriers for patients may present implementation barriers.

\section{Research recommendation}

Further methodologically rigorous trials in women with polycystic ovary syndrome are important to address:

1 The types of diet delivery methods and diet support and tools that are effective - an initial study would be to have diet compositions the same and vary the delivery method or support tools between the groups

2 Comparison of the delivery methods for lifestyle interventions including comparing settings and providers.

To find a dietitian, follow the links on the Dietitians Association of Australia website: http://www.daa.asn.au.

Clinical question. In women with PCOS, are exercise interventions (compared to no exercise or different exercises) effective for improving weight loss, metabolic, reproductive, fertility, quality of life and emotional wellbeing outcomes?

Recommendations

Evidence-based recommendation

5.5a Exercise participation of at least 150 minutes per week should be recommended to all women with polycystic ovary syndrome, especially those with a body mass index $\geqslant 25 \mathrm{~kg} / \mathrm{m}^{2}$ (overweight), given the metabolic risks of polycystic ovary syndrome and the long-term metabolic benefits of exercise. Of this, 90 minutes per week should be aerobic activity at moderate to high intensity ( $60 \%-90 \%$ of maximum heart rate) to optimise clinical outcomes. - level D

\section{Clinical practice point}

5.5b Encouraging exercise is the joint responsibility of all health professionals, partnering with women with polycystic ovary syndrome. Where appropriate, referral to an exercise physiologist or specialist could be considered as part of an enhanced primary care plan. Where there are significant comorbidities, assessment for exercise participation should be undertaken by the relevant health care professionals.

Tools such as Lifescripts (http://www.health.gov.au/ lifescripts) could be used for engagement in dietary change.

Context. Exercise effectively ameliorates IR and provides an effective intervention for management of PCOS. Moderate aerobic exercise can be defined as an intensity between $50 \%-80 \%$ of maximum oxygen consumption or $60 \%-90 \%$ of maximal heart rate (Figure 9). ${ }^{181}$ A single session of moderate exercise enhances glucose disposal and improves insulin sensitivity. ${ }^{182}$ Ongoing moderate exercise, at least three to five times per week, consistently reduces DM2 risk $^{48,183}$ and improves CVD risk factors. ${ }^{184,185}$ Similarly, resistance or weight-bearing exercise alone or in combination with aerobic exercise improves health outcomes in high-risk groups. ${ }^{186-189}$ There is significant evidence to support exercise alone, independent of weight loss; ${ }^{160,190}$ but the intensity, type and frequency of exercise remains unclear; hence, this clinical question was prioritised during guideline development.

Evidence informing the recommendations. Eight RCTs with a high risk of bias were identified by the search to answer this question. Two studies compared different exercises. ${ }^{140,191,192}$ Orio and colleagues showed that 24 weeks' exercise was better than no exercise with diet for fasting glucose, insulin area under the curve (AUC), glucose-insulin AUC ratio, LDL-C, HDL-C, BMI, waisthip ratio, and waist circumference. ${ }^{191}$ However, evidence was inconsistent among the five studies that compared exercise to no exercise. ${ }^{193-197}$ Exercise was better than no exercise for triglycerides; ${ }^{193}$ BMI $(P=0.05) ;{ }^{196}$ fasting insulin $(P<0.01)$; insulin AUC $(P<0.001)$; glucose-insulin AUC ratio $(P<0.001)$; highly sensitive $C$-reactive protein $(P<0.01)$; SHBG $(P<0.05)$; BMI $(P<0.05)$; waist-hip ratio $(P<0.05) ;{ }^{195}$ glucose-insulin AUC ratio $(P<0.001)$; systolic blood pressure $(P<0.01)$; and diastolic blood pressure at peak exercise $(P<0.01){ }^{197}$

One study found that exercise was better for menses frequency $(P=0.04)$; increased ovulation rate $(P=0.03)$; and increased cumulative ovulation rate $(P=0.01){ }^{164}$ There was no difference between aerobic exercise and aerobic-resistance exercise for percent of body fat, fat mass, fat-free mass, depression scores and PCOS-related QoL. ${ }^{140,192}$ Overall, although exercise has benefits for women with PCOS who are overweight, the evidence is limited, inconsistent and of low quality.

The evidence from the included studies is directly generalisable to women with PCOS who are overweight, with practical exercise regimens used. There may be limitations to generalising these findings to other ethnic groups, especially to non-European populations.

Implications of the recommendations. Health care professionals can recommend 150 minutes of exercise per week based on current national and international guidelines for the general population ${ }^{198-206}$ on exercise 
Figure 9. Intensities of different types of exercise activities ${ }^{200}$

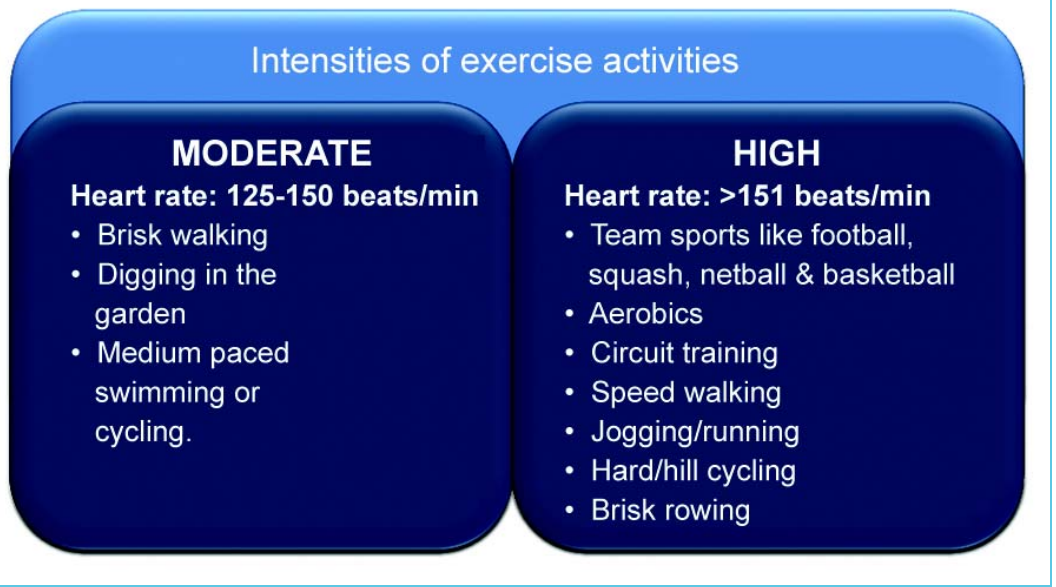

prescription for health and weight maintenance. Within this, 90 minutes of moderate- to high-intensity exercise (Figure 9) can be recommended. Where there are barriers to exercise, consider involving an exercise physiologist to help with structured exercise training. For most practices, this recommendation will change usual care, reduce variation in practice and optimise exercise prescription and may result in increased referral to exercise physiologists. There may initially be extra costs to the health care system. Problems with engagement of, and adequate access to, health professionals, including insufficient consultation time with GPs, may be barriers. Drop-out from or nonadherence to exercise programs continues to be a problem, but if there is adequate allied health and clinical support, these challenges may be reduced. A shift in cultural mindset may be required to ensure clinicians and women with PCOS understand the importance of exercise in PCOS management.

Research recommendation

Further methodologically rigorous trials in women with polycystic ovary syndrome are important to help define what types, intensities and delivery methods of exercise strategies are optimal for lean and overweight women for improved clinical outcomes.

To find an exercise physiologist, follow the links on the Exercise and Sports Science Australia website (http:// www.essa.org.au). 


\section{Section 6. Non-pharmacological first-line management of infertility in PCOS (Algorithms 4 and 5)}

\begin{abstract}
Clinical question. In women with PCOS, what is the effectiveness of lifestyle interventions compared with pharmacological interventions (ie, metformin and clomiphene citrate) for improving fertility, quality of life and emotional wellbeing outcomes?

Recommendations

Evidence-based recommendations

6.1a Lifestyle management, including diet and exercise programs, should be used throughout the life span in women with polycystic ovary syndrome to optimise health generally and to alleviate polycystic ovary syndrome clinical severity including infertility. - level C

6.1b In women with polycystic ovary syndrome and body mass index $\geqslant 30 \mathrm{~kg} / \mathrm{m}^{2}$ with due consideration given to age-related infertility, intensive (frequent multidisciplinary contact) lifestyle modification alone (and not in combination with pharmacological ovulation induction therapy) should be first line therapy for 3 to 6 months to determine if ovulation is induced. - level C

6.1c Pharmacological ovulation induction should not be recommended for first line therapy in women with polycystic ovary syndrome who are morbidly obese (body mass index $\geqslant 35 \mathrm{~kg} / \mathrm{m}^{2}$ ) until appropriate weight loss has occurred either through diet, exercise, bariatric surgery, or other appropriate means. - level C

6.1d Pharmacological ovulation induction could be second line therapy, after intensive lifestyle modification has been undertaken. - level C
\end{abstract}

Clinical practice points

6.le Morbid obesity (body mass index $\geqslant 35 \mathrm{~kg} / \mathrm{m}^{2}$ ) increases risks during pregnancy and should be regarded as a relative contraindication to assisted fertility.

6.If Psychological factors should be considered and managed in infertile women with polycystic ovary syndrome, to optimise engagement and adherence with lifestyle interventions.

Context. Lifestyle therapy is deemed first line for women with PCOS, targeting prevention of weight gain and promoting weight loss where required, with significant health benefits. ${ }^{6,163,207}$ Fertility rates are lower among women with a BMI $\geqslant 30-32 \mathrm{~kg} / \mathrm{m}^{2}$, and lifestyle intervention benefits extend well beyond fertility and include DM2 prevention. There remains inadequate highquality evidence on pregnancy outcomes with lifestyle intervention in PCOS. ${ }^{163}$ Pharmacological therapy including clomiphene citrate, metformin and gonadotrophins are available and effective in improving fertility in women with PCOS. ${ }^{208}$ However, there is little comparative data on the benefits of lifestyle versus pharmacological therapy in women with PCOS who are overweight or obese; hence, this clinical question was prioritised during guideline development.

Evidence informing the recommendations. Ten RCTs (level II), of moderate ${ }^{157,209,210}$ to high ${ }^{145,154,155,211-214}$ risk of bias compared various types of lifestyle interventions (including low-carbohydrate or healthy diets, exercise programs and group sessions) to pharmacological therapy (including metformin or clomiphene citrate) among women with PCOS and a BMI $\geqslant 25 \mathrm{~kg} / \mathrm{m}^{2}$. There was little difference between lifestyle (diet) therapy (with or without placebo) and pharmacological therapy (with or without lifestyle [diet] therapy) for fertility outcomes. Gambineri and colleagues reported that metformin was better than placebo plus diet for menstrual pattern after $6(P=0.03)$ and 12 months $(P=0.003),{ }^{145,211}$ and found a change in frequency of menstruation from baseline to 12 months $(P=$ 0.01). Pasquali and colleagues reported that metformin plus diet was better than placebo plus diet $(P<0.05)$ for menstruation frequency. ${ }^{154}$ Another study reported that lifestyle plus clomiphene citrate was better than lifestyle alone for ovulation rate and menstrual frequency $(P<0.05){ }^{210}$

Adverse events were not consistently reported. Two patients had adverse events in the metformin group, ${ }^{212}$ and Qublan and colleagues reported that 11 women withdrew due to side effects in the metformin plus diet group. ${ }^{155}$ Six women withdrew from the placebo plus diet group due to undefined side effects within the first 2 months of the study. ${ }^{155}$ It is important to note that there was inconsistency in the components of lifestyle (diet) therapy, so a specific intervention cannot be recommended; however, given the similar efficacy of lifestyle and pharmacological therapy overall, the guideline development group deemed clear benefits in using lifestyle interventions over pharmacological interventions for firstline management of infertility among women with PCOS.

Implications of the recommendations. Lifestyle management, including diet and exercise programs, should be used throughout the life span in women with PCOS to optimise health generally and to improve fertility outcomes. Pharmacological ovarian stimulation and/or assisted fertility should not be prescribed to women who are morbidly obese $\left(\mathrm{BMI} \geqslant 35 \mathrm{~kg} / \mathrm{m}^{2}\right.$ ) unless appropriate weight loss has occurred, in accordance with the Royal Australian and New Zealand College of Obstetricians and Gynaecologists recommendations. ${ }^{215}$ With lifestyle intervention as first line for women with PCOS who are overweight, initial therapy for infertility can be commenced in primary care. Barriers to implementation include impact on workloads in general practice, increased referral to allied health professionals and availability and funding for lifestyle programs. A shift in cultural mindset may be required to focus on lifestyle interventions, especially around prevention of weight gain.

\section{Research recommendation}

Further methodologically rigorous trials are important to investigate the impact of lifestyle improvement on fertility outcomes including live birth. Implementation research into the optimal engagement, adherence and delivery of lifestyle intervention is needed to inform consumers, health professionals and policy makers. 


\section{Section 7. Pharmacological management of infertility in PCOS (Figure 10) (Algorithms 4 and 5)}

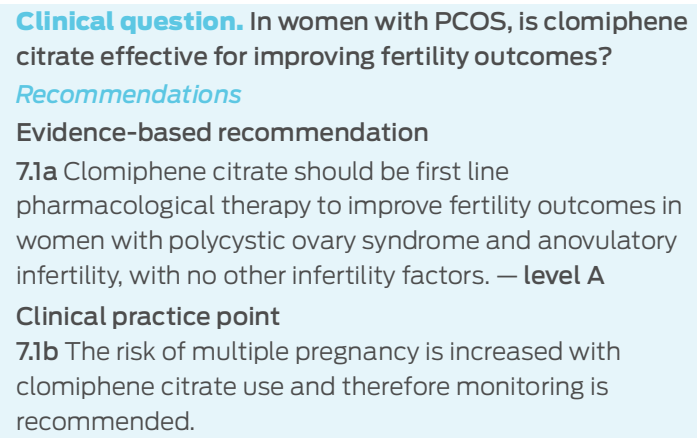

Context. Clomiphene citrate is a selective oestrogen receptor modulator with both oestrogenic and antioestrogenic properties. ${ }^{216}$ It was first approved for management of anovulation in 1967 it has been a first-line ovulation-induction agent for over 40 years. It inhibits oestradiol receptors binding in the hypothalamus and pituitary, blocking negative feedback. This hypothalamus release from negative inhibition increases gonadotrophinreleasing hormone, which in turn increases FSH and stimulates follicular growth and ovulation. ${ }^{217}$

Standard practice is to titrate clomiphene citrate doses up to $150 \mathrm{mg} /$ day if ovulation is not achieved, at which point clomiphene citrate resistance (CCR) is reached. If ovulation is not achieved after six ovulatory cycles with clomiphene citrate, this is termed a state of clomiphene citrate failure. ${ }^{218}$ Studies with clomiphene citrate show ovulation rates of $60 \%-85 \%$ and pregnancy rates of $30 \%-$ $50 \%$ after six ovulatory cycles. The discrepancy between ovulation rates and pregnancy rates has been attributed to anti-oestrogenic clomiphene citrate effects. The rates of twin and triplet pregnancy with clomiphene citrate are $5 \%-7 \%$ and $0.3 \%$, respectively. The incidence of ovarian hyperstimulation syndrome is less than $1 \%{ }^{219}$ It is best to limit lifetime exposure to 12 treatment cycles, with concerns of increased risk of borderline ovarian tumours with longer exposure. ${ }^{220}$ Comparative effectiveness of clomiphene citrate among infertile women with PCOS needs to be established, especially in light of potential risks.

Evidence informing the recommendations. One highquality systematic review (level I) with low risk of bias found that clomiphene citrate was better than placebo for pregnancy rate per patient $(P<0.009)$ and ovulation rate per patient $(P<0.001)$ in women with PCOS; however, this included participants whose sensitivity to clomiphene citrate was not reported. ${ }^{221}$ The evidence obtained from this systematic review is generalisable to the patient population in terms of age and BMI. The setting was varied and study locations included the United States and Canada. None of the studies were conducted in Australia and may not be generalisable to Indigenous populations.

Implications of the recommendations. First-line clomiphene citrate therapy should be prescribed in specialist care. It should be noted that there are limitations, which differ between Australian states, in terms of which

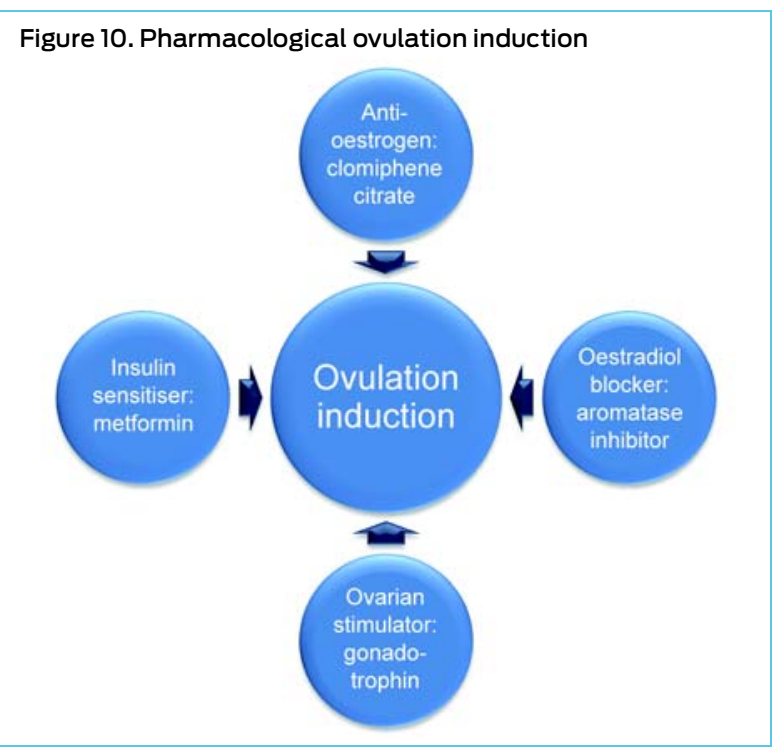

specialists are able to prescribe and monitor clomiphene citrate therapy. There may be resource implications as adequate monitoring will require additional resources. Costs to the patient of having adequate monitoring (tests and specialist visits) and accessibility to specialist care may be barriers to the use of clomiphene citrate; however, it is anticipated that increased costs will be offset by reduced multiple pregnancy-related costs.

Clinical question. In women with PCOS, is metformin effective for improving fertility outcomes?

Recommendations

Evidence-based recommendations

7.2a Metformin should be combined with clomiphene citrate to improve fertility outcomes rather than persisting with further treatment with clomiphene citrate alone in women with polycystic ovary syndrome who are clomiphene citrate resistant, anovulatory and infertile with no other infertility factors. - level A

7.2b Metformin could be used alone to improve ovulation rate and pregnancy rate in women with polycystic ovary syndrome who are anovulatory, have a body mass index $\leqslant 30 \mathrm{~kg} / \mathrm{m}^{2}$ and are infertile with no other infertility factors. - level B

$7.2 \mathrm{c}$ If one is considering using metformin alone to treat women with polycystic ovary syndrome who are anovulatory, have a body mass index $\geqslant 30 \mathrm{~kg} / \mathrm{m}^{2}$, and are infertile with no other infertility factors, clomiphene citrate should be added to improve fertility outcomes. - level A

Context. IR leading to hyperinsulinaemia increases ovarian androgen biosynthesis and decreased SHBG synthesis, further increasing free androgens. Excess androgens and insulin cause premature follicular atresia and anovulation. These mechanistic insights have led to the use of insulin-sensitising drugs in PCOS. Metformin has been used extensively in DM2, is the most studied in PCOS, and has the most reassuring safety profile. ${ }^{222}$ 
Studies examining the reproductive effects of metformin in PCOS suggest benefits but are limited by study design. 223 Metformin regimens in PCOS are not well standardised in research or clinical practice, with doses varying between 500 and $2550 \mathrm{mg}$ per day and both standard and slowrelease preparations available. ${ }^{209}$

Evidence informing the recommendations.

Metformin versus placebo: Forty-four articles met selection criteria, with the most current, high-level and high-quality evidence used. Three systematic reviews (two high-quality systematic reviews [level I] with low risk of bias $^{208,224}$ and a medium-quality systematic review [level I] with moderate risk of bias ${ }^{225}$ ) and as one high-quality RCT [level II] with low risk of bias (that superseded the recent systematic review) ${ }^{226}$ were appraised. The findings in women with PCOS are presented in Box 2.

Metformin versus clomiphene citrate: Two systematic reviews (one high-quality systematic review [level I] with low risk of bias ${ }^{208}$ and a medium-quality systematic review [level I] with moderate risk of bias ${ }^{225}$ ) and two RCTs (one high-quality RCT [level II] with low risk of bias ${ }^{226}$ and a medium-quality RCT [level II] with moderate risk of bias ${ }^{213}$ (that superseded the recent systematic review) were appraised. The findings for women with PCOS are presented in Box 3.

Metformin plus clomiphene citrate versus clomiphene citrate alone: Three systematic reviews (two high-quality systematic reviews [level I] with low risk of bias ${ }^{208,224}$ and a medium-quality systematic review [level I] with moderate risk of bias ${ }^{225}$ ) and five RCTs (three high-quality RCT [level II] with low risk of bias, ${ }^{226-228}$ a medium-quality RCT [level II] with moderate risk of bias ${ }^{213}$ and a low-quality RCT [level II] with high risk of bias ${ }^{229}$ ) (all RCTs superseded the most current systematic review) were appraised. The findings in women with PCOS are presented in Box 4.

Metformin plus clomiphene citrate versus metformin alone: One high-quality systematic review (level I) with low risk of bias evaluating two RCTs with a mean BMI $\geqslant 30 \mathrm{~kg} / \mathrm{m}^{2,230}$ and two RCTs (one high-quality RCT [level II] with low risk of bias ${ }^{226}$ and one medium-quality RCT [level II] with moderate risk of bias ${ }^{213}$ ) (both RCTs superseded recent systematic review). The findings in women with PCOS are presented in Box 5.

The evidence is generalisable to the patient population in terms of age and BMI and was completed in multiple countries; however, it may not be generalisable to Indigenous populations.

Implications of the recommendations. Metformin should be added to clomiphene citrate in women with CCR PCOS; metformin alone could be used in women with PCOS and a BMI $\leqslant 30 \mathrm{~kg} / \mathrm{m}^{2}$; and clomiphene citrate should be added to metformin for women with PCOS and a BMI $\geqslant 30 \mathrm{~kg} / \mathrm{m}^{2}$. This may result in a change in usual care as clinicians may now be more likely to prescribe metformin. Metformin may be associated with mild gastrointestinal adverse events and women should be informed about these. The guideline development group is not aware of resource implications or changes to the way care is organised with implementation of the recommendations.
One barrier may be that although metformin is listed as an unrestricted benefit on the Pharmaceutical Benefits Scheme, no application for metformin use in PCOS has been made to the Australian Therapeutic Goods Administration (TGA) and hence it is not approved for this indication. For TGA approval, industry must propose metformin for approval, and as metformin is generic and produced by many companies, it is unlikely that a company will fund and support an application to the TGA. This technical challenge is independent of effectiveness or evidence. Although use is evidence-based, patient explanation and consent is appropriate.

\section{Research recommendation}

Further methodologically rigorous trials are important to address:

1 Whether the addition of metformin to clomiphene citrate improves live birth rate in anovulatory women with PCOS and no other infertility factors

2 Whether there is a difference in effectiveness between clomiphene citrate and metformin in women with PCOS who are anovulatory and infertile with a body mass index $\leqslant 30 \mathrm{~kg} / \mathrm{m}^{2}$ to improve fertility outcomes.

Clinical question. In women with PCOS, are gonadotrophins effective for improving fertility outcomes?

Recommendations

Evidence-based recommendations

7.3a Gonadotrophins should be second line pharmacological therapy in women with polycystic ovary syndrome who have clomiphene citrate resistance and/or failure, are anovulatory and infertile, with no other infertility factors. - level B

7.3b Gonadotrophins could be considered as first line pharmacological therapy in women with polycystic ovary syndrome who are therapy naive, anovulatory and infertile, with no other infertility factors. - level C

Clinical practice point

7.3c Where gonadotrophins or laparoscopic ovarian

surgery (see 8.1) are to be prescribed, the following should be considered:

- Cost of either intervention for ovulation induction

- Expertise required for the use of either intervention for ovulation induction

- The degree of intensive monitoring that is required for gonadotrophin therapy

- Implications of potential multiple pregnancy for gonadotrophin therapy

- Implications of the potential risk of ovarian hyperstimulation syndrome for gonadotrophin therapy

- Laparoscopic surgery in women who are overweight or obese is associated with both intra-operative and postoperative risks.

Context. Gonadotrophins are often used as second-line therapy in anovulatory PCOS women with CCR; however, there is an increased risk of multiple pregnancy and ovarian hyperstimulation syndrome. ${ }^{231}$ A low-dose "stepup" protocol is used to reduce these risks. ${ }^{232}$ A "stepdown" protocol can also be used ${ }^{233}$ with similar success, but is not recommended for routine use. Despite a large body of observational evidence supporting the use of gonadotrophin therapy in anovulatory women with 
Box 2. Metformin versus placebo in polycystic ovary syndrome (PCOS)

Ovulation rates: Metformin outperformed placebo among overall women with PCOS (defined as all the women with PCOS in the relevant study or studies, regardless of body mass index [BMI] or clomiphene citrate exposure or sensitivity) $(P<0.001)$, among women with $P C O S$ and with a $\mathrm{BMl} \leqslant 30 \mathrm{~kg} / \mathrm{m}^{2}(P<0.001)$, with $\mathrm{a} \mathrm{BMI} \geqslant 30 \mathrm{~kg} / \mathrm{m}^{2}(P=0.007), 208$ and among women with nonclomiphene citrate-resistant (CCR) PCOS (defined as clomiphene citrate sensitive or unknown clomiphene citrate sensitivity).224 There was significant statistical heterogeneity among overall women with PCOS $\left(1^{2}=69 \%\right)$ and among those with a BMI $\leqslant 30 \mathrm{~kg} / \mathrm{m}^{2}\left(\mathrm{I}^{2}=88 \%\right)$. Ovulation rates were similar between groups in CCR women.

Pregnancy rates: Metformin outperformed placebo among overall women with PCOS $(P<0.001)$, without statistical heterogeneity $\left(\mathrm{I}^{2}=0\right)$ and among those with a $\mathrm{BMI} \leqslant 30 \mathrm{~kg} / \mathrm{m}^{2}(P<0.001)$ with little statistical heterogeneity $\left(I^{2}=40 \%\right)^{208}$ but no difference was noted in those with $\mathrm{BMI} \geqslant 30 \mathrm{~kg} / \mathrm{m}^{2}$, in clomiphene citrate-naive women, women with CCR PCOS or women with non-CCR PCOS.

Live birth rates: There was no difference between metformin and placebo in overall women with PCOS, in women with CCR and women with $\mathrm{BMI} \geqslant 30 \mathrm{~kg} / \mathrm{m}^{2}$.

Miscarriage rates: There was no difference between metformin and placebo in overall women with PCOS.

Adverse effects: Metformin induced more gastrointestinal-related adverse events compared with placebo $(P<0.001)$, with little statistical heterogeneity $\left(I^{2}=25 \%\right){ }^{208}$

PCOS, ${ }^{232}$ its comparative effectiveness needs to be evaluated using the best available evidence.

Evidence informing the recommendations. One systematic review and one RCT were identified by our search. There was no difference in effectiveness between gonadotrophins and laparoscopic ovarian surgery. The high-quality RCT (level II) with low risk of bias found that in therapy-naive women with PCOS, FSH and clomiphene citrate were similar for all fertility outcomes; ${ }^{234}$ however, with small sample size and inadequate power, this cannot inform a recommendation in isolation.

A multicentre RCT on first-line clomiphene citrate versus gonadotrophins in therapy-naive anovulatory women with PCOS reported higher clinical pregnancy rate with gonadotrophins. Pregnancy rates with gonadotrophin therapy were almost double in the first cycle compared with clomiphene citrate. ${ }^{235}$ However, this study is only published in abstract form, informing the guideline development group but not included in the evidence review.

The evidence is generalisable to patient populations in terms of age and BMI and applicability in Australia, but may not be relevant to Indigenous populations.

Implications of the recommendations. Gonadotrophins are suitable for second-line therapy for improving infertility in women with PCOS in specialist care. The guideline development group is not aware of resource implications or barriers to implementation. Gonadotrophins appear to be as effective as laparoscopic ovarian surgery and clinical practice points should be considered in deciding between the two options.

\section{Research recommendation}

Further methodologically rigorous trials are important to address the role of gonadotrophins in ovulation induction in polycystic ovary syndrome.
Box 3. Metformin versus clomiphene citrate in polycystic ovary syndrome (PCOS)

Ovulation rates: Clomiphene citrate outperformed metformin among overall women with PCOS (defined as all the women with PCOS in the relevant study or studies, regardless of body mass index $[\mathrm{BMI}])(P<0.001)$ and among those with a $\mathrm{BMI} \geqslant 30 \mathrm{~kg} / \mathrm{m}^{2}$ $(P<0.001){ }^{208}$ Statistical heterogeneity was significant among overall women with PCOS $\left(1^{2}=78 \%\right)$. Clomiphene citrate was comparable to metformin among those with a $\mathrm{BMI} \leqslant 30 \mathrm{~kg} / \mathrm{m}^{2}$. Pregnancy rates: $\mathrm{Clomiphene} \mathrm{citrate} \mathrm{outperformed} \mathrm{metformin}$ among overall women with PCOS $(P=0.018)$ and among those with a $\mathrm{BMI} \geqslant 30 \mathrm{~kg} / \mathrm{m}^{2}(P<0.001)$. However, there was significant statistical heterogeneity seen in overall women with PCOS $\left(1^{2}=\right.$ $91 \%$ ). Metformin outperformed clomiphene citrate among those with a $\mathrm{BMI} \leqslant 30 \mathrm{~kg} / \mathrm{m}^{2}$; however, this was based on a single study $(P=0.003){ }^{208}$

Live birth rates: Clomiphene citrate and metformin were comparable in overall women with PCOS, with statistical heterogeneity. Clomiphene citrate was better than metformin for live birth rate in those with a BMI $\geqslant 30 \mathrm{~kg} / \mathrm{m}^{2}(P<0.002)$ without statistical heterogeneity $\left(I^{2}=0\right)$. Metformin outperformed clomiphene citrate in those with a $\mathrm{BMI} \leqslant 30 \mathrm{~kg} / \mathrm{m}^{2}$; however, this was based on a single study $(P<0.001){ }^{208}$

Adverse effects: There was no difference in multiple pregnancy rate (without statistical heterogeneity) and miscarriage rate (with statistical heterogeneity) between metformin and clomiphene citrate in overall women with PCOS.

Clinical question. In women with PCOS, are aromatase inhibitors effective for improving fertility outcomes?

Recommendations

Evidence-based recommendations

7.4a Letrozole should not be first line pharmacological therapy in women with polycystic ovary syndrome who are anovulatory, and infertile, with no other infertility factors. level $B$

7.4b Under caution either letrozole or anastrozole could be used if one is considering using aromatase inhibitors in women with polycystic ovary syndrome women who are clomiphene citrate resistant, anovulatory and infertile with no other infertility factors. If using letrozole, it is preferable to treat for 10 days at a dose of $2.5 \mathrm{mg} /$ day. - level $D$

Context. Aromatase inhibitors were proposed as ovulation-inducing agents in 2001. ${ }^{236}$ Aromatase converts androgens to oestrogens, and aromatase inhibitors therefore inhibit oestrogen biosynthesis, interfering with hypothalamus/pituitary feedback, increasing FSH and causing ovarian follicle stimulation. ${ }^{237}$ These agents theoretically avoid adverse effects of clomiphene citrate, $^{238,239}$ as they do not affect oestrogen receptors centrally or thin the endometrium. ${ }^{237}$

Adverse effects include gastrointestinal disturbances, asthenia, hot flushes, headache and back pain. ${ }^{237}$ The potential for teratogenic effects of letrozole has been raised, with an abstract suggesting higher risk of congenital cardiac and bone malformations in newborns. $^{240}$ However, two subsequent publications suggest that letrozole may not be associated with fetal anomalies. ${ }^{241,242}$ Given their current use in ovulation induction with limited data and potential adverse effects, this clinical question was prioritised by the guideline development group.

Evidence informing the recommendations. Our search did not identify any studies addressing the effectiveness of letrozole as first-line therapy in PCOS (ie, letrozole versus 
Box 4. Metformin plus clomiphene citrate versus clomiphene citrate alone in polycystic ovary syndrome (PCOS)

Ovulation rates: Metformin plus clomiphene citrate was better than clomiphene citrate alone among overall women with PCOS (defined as all the women with PCOS in the relevant study or studies, regardless of body mass index [BMI] $)(P<0.001)$ and among those with a BMI $\leqslant 30 \mathrm{~kg} / \mathrm{m}^{2}(P=0.009)$, with a BMI $\geqslant 30 \mathrm{~kg} / \mathrm{m}^{2}(P<0.001)$, with clomiphene citrate-resistant (CCR) PCOS $(P<0.001), 208$ with non-CCR PCOS, 224 and where CCR status was unknown $(P<0.001) .208$ However, there was significant statistical heterogeneity in these group comparisons $\left(I^{2}>65 \%\right)$, except among women with CCR PCOS $\left(I^{2}=0\right)$. There was no difference between the two treatments in women with clomiphene citrate-sensitive PCOS.

Pregnancy rates: Metformin plus clomiphene citrate was better than clomiphene citrate alone among overall women with PCOS $(P=0.006)$, among those with a $\mathrm{BMI} \geqslant 30 \mathrm{~kg} / \mathrm{m}^{2}(P=0.004){ }^{208}$ clomiphene citrate-naive women $(P<0.001)$, and women with CCR PCOS $(P<0.001) .225$ There was significant statistical heterogeneity in all these group comparisons $\left(I^{2}>58 \%\right)$ except among those with $\mathrm{a} B M I \geqslant 30 \mathrm{~kg} / \mathrm{m}^{2}\left(1^{2}=40 \%\right)$ and those with CCR PCOS $\left(1^{2}=0\right)$.

There was no difference between metformin plus clomiphene citrate and clomiphene citrate alone among those with a BMI $\leqslant 30 \mathrm{~kg} / \mathrm{m}^{2}$, non-CCR PCOS, and those with unknown clomiphene citrate sensitivity.

Live birth rates: Metformin plus clomiphene citrate was better than clomiphene citrate alone for increasing the live birth rate among women with CCR PCOS $(P=0.03),{ }^{225}$ without statistical heterogeneity $\left(I^{2}=0\right)$. Live birth rate was similar between the two groups overall, in those with a $\mathrm{BMl} \leqslant 30 \mathrm{~kg} / \mathrm{m}^{2}$, a BMl $\geqslant 30 \mathrm{~kg} / \mathrm{m}^{2}$ and clomiphene citrate-naive PCOS. Miscarriage rates were similar in overall women with PCOS and in those with a BMI $\leqslant 30 \mathrm{~kg} / \mathrm{m}^{2}$. Likewise, multiple pregnancy rates were similar in overall women with $\mathrm{PCOS}$, in those with a $\mathrm{BMI} \leqslant 30 \mathrm{~kg} / \mathrm{m}^{2}$ and in those with unknown clomiphene citrate sensitivity.

Adverse effects: Clomiphene citrate alone had fewer gastrointestinal adverse events compared with metformin plus clomiphene citrate.

placebo in treatment-naive patients). We identified 11 studies on letrozole as second-line therapy in PCOS. One high-quality RCT (level II) with a low risk of bias using letrozole versus placebo in PCOS with CCR, found letrozole better for ovulation rates $(P=0.006)$ but similar for pregnancy rate and live birth rate per patient. ${ }^{243}$

Two RCTs (level II), one with a high risk of bias ${ }^{244}$ and one with a low risk of bias, ${ }^{245}$ found no difference between letrozole and anastrozole for ovulation and pregnancy rate per cycle and miscarriage rate per pregnancy among women with CCR PCOS. ${ }^{245}$ The lower-quality study ${ }^{244}$ found letrozole to be better; however, results should be interpreted with caution.

One high-quality RCT (level II) with low risk of bias found 10 days of letrozole better than 5 days for pregnancy rate per cycle $(P=0.03)$, with no differences in ovulation rate per patient and miscarriage rate per pregnancy among women with CCR PCOS. ${ }^{246}$

Six RCTs (level II) of low, ${ }^{247,248}$ moderate 249,250 and high risk of bias ${ }^{251,252}$ compared letrozole to clomiphene citrate. Meta-analysis of these RCTs revealed that letrozole was
Box 5. Metformin plus clomiphene citrate versus metformin alone in polycystic ovary syndrome (PCOS)

Ovulation rates: Metformin plus clomiphene citrate was better than metformin alone among overall women with PCOS (defined as all the women with PCOS in the relevant study or studies $(P<0.001){ }^{230}$

Pregnancy rates: Metformin plus clomiphene citrate was better than metformin alone among overall women with PCOS $(P<0.001)^{230}$

Live birth rates: Metformin plus clomiphene citrate was better than metformin alone in overall women with PCOS $(P<0.001)$. $^{230}$

Adverse effects: There was no difference between metformin plus clomiphene citrate and metformin alone for miscarriage rate or adverse events in overall women with PCOS.

better than clomiphene citrate for ovulation rate per patient after combining three studies $(P<0.0001)^{249-251}$ of women with therapy-naive CCR PCOS (although some were without clarification on CCR status). Meta-analyses of two other studies showed no differences in ovulation rate per cycle ${ }^{247,248}$ in therapy-naive women with PCOS, ${ }^{247}$ and women with PCOS where CCR status was not reported. ${ }^{248}$ Further, no differences in pregnancy rate per patient, ${ }^{247-251}$ miscarriage and live birth rate per pregnancy and multiple pregnancy rate per patient were noted between letrozole and clomiphene citrate. ${ }^{247-251}$

One medium-quality RCT (level II) with moderate risk of bias compared letrozole to clomiphene citrate plus metformin. No difference in ovulation and pregnancy rate per cycle or miscarriage and multiple pregnancy rate per pregnancy was noted in CCR PCOS. ${ }^{253}$

The evidence was generalisable in terms of age and BMI and studies were conducted in many nations. They may be applicable in Australia, but may not be relevant to Indigenous populations.

Implications of the recommendations. We recommend caution with aromatase inhibitor use, as these agents have not been approved for use in ovulation induction in PCOS. The Pharmaceuticals Benefits Scheme does not reimburse costs and it is unlikely these agents would be first-line routine treatment in PCOS. The evidence about congenital anomalies with aromatase inhibitors is unclear and should prompt patient explanation and consent. The guideline development group is not aware of resource implications, changes to the way care is organised or barriers to implementation of these recommendations.

Research recommendations

Further methodologically rigorous trials are important to address the role of aromatase inhibitors in ovulation induction in polycystic ovary syndrome.

Further methodologically rigorous trials are important to address the impact of different ovulation induction agents in subgroups of polycystic ovary syndrome including those based on BMI. 


\section{Section 8. Surgical management of infertility in PCOS (Algorithms 4 and 5)}

Clinical question. In women with PCOS, is ovarian surgery effective for improving fertility outcomes? Recommendations

Evidence-based recommendation

8.1a Laparoscopic ovarian surgery should be second line therapy in women with polycystic ovary syndrome who are clomiphene citrate resistant, anovulatory, and infertile, with no other infertility factors. - level B

Clinical practice point

8.1b If undergoing laparoscopic ovarian surgery, the patient should be advised of the risks (see below).

Clinical consensus recommendation

8.1c Where ovulation induction would be considered appropriate, laparoscopic ovarian surgery can be used as first line treatment if laparoscopy is indicated for another reason in infertile women with PCOS.

Clinical practice point

8.1d Where gonadotrophins (see 7.3) or laparoscopic ovarian surgery are to be prescribed, the following should be considered:

- Cost of either intervention for ovulation induction

" Expertise required for the use of either intervention for ovulation induction

" The degree of intensive monitoring that is required for gonadotrophin therapy

- Implications of potential multiple pregnancy for gonadotrophin therapy

- Implications of the potential risk of ovarian hyperstimulation syndrome for gonadotrophin therapy - Laparoscopic surgery in women who are overweight or obese are associated with both intraoperative and postoperative risks.

Context. Laparoscopic ovarian "drilling" was first described in $1984^{254}$ with minor variations developed subsequently; all are characterised by an altered endocrine profile following surgery. Mechanisms underpinning hormonal changes and resumption of ovulation remain poorly understood. Laparoscopic ovarian drilling in PCOS is thought

- to cause fewer adhesions than performing a laparotomy;

- to be more cost-effective as an outpatient procedure; and

- to restore regular mono-ovulations, albeit for a limited time in most patients.

In contrast, ovulation induction with gonadotrophins is expensive and requires regular monitoring, with risks of multiple pregnancy and ovarian hyperstimulation syndrome. The mechanisms of action, comparative effectiveness and the long-term risks of laparoscopic ovarian surgery in infertile women with PCOS still remain unclear; hence, this area has been prioritised in guideline development.

Evidence informing the recommendations. Six articles reporting five studies were identified by our search to answer this question. One high-quality systematic review of RCTs (level I) with low risk of bias compared laparoscopic ovarian surgery to gonadotrophins with no differences for live birth rate, pregnancy and ovulation rate per patient and miscarriage rate per pregnancy, but laparoscopic ovarian surgery had fewer multiple pregnancies. $^{255}$

One high-quality RCT (level II) with a low risk of bias found that clomiphene citrate plus metformin was better than laparoscopic ovarian surgery for ovulation rate per cycle $(P=0.02)$, but there was no difference for live birth rate, pregnancy rate per cycle and miscarriage rate per pregnancy. ${ }^{256}$

Two medium-quality RCTs (level II) with moderate risk of bias found insufficient evidence to recommend laparoscopic ovarian surgery compared with metformin for live birth rate per patient, ovulation or pregnancy rate per cycle, pregnancy rate per patient and miscarriage rate per pregnancy. ${ }^{257-259}$ One reported laparoscopic ovarian surgery was better than metformin for ovulation $(P=0.001)$ and pregnancy rate per cycle and per patient $\left(P=0.03\right.$ and $P=0.03$, respectively), ${ }^{257}$ and the other reported metformin was better than laparoscopic ovarian surgery for pregnancy rate per cycle $(P<0.05)$, and miscarriage rate $(P<0.05){ }^{258-259}$

One high-quality RCT (level II ${ }^{260}$ and one high-quality systematic review of RCTs (level I), ${ }^{255}$ both with low risk of bias, found that laparoscopic ovarian surgery was better than gonadotrophins for multiple pregnancy rate, ${ }^{255}$ and that there was no difference between laparoscopic ovarian surgery and clomiphene citrate for live birth rate per patient, pregnancy rate per patient, ovulation rate per patient and miscarriage rate per pregnancy. ${ }^{260}$

There was insufficient evidence to support or refute the use of laparoscopic ovarian surgery over either metformin or clomiphene citrate alone, or clomiphene citrate plus metformin, for multiple pregnancies, ${ }^{257,260}$ and there was insufficient evidence to support or refute the use of laparoscopic ovarian surgery over any intervention for adverse effects and QoL.

The evidence was from multiple countries and may be generalisable to the patient population in terms of age and BMI. The RCT ${ }^{261}$ included in the systematic review by Farquhar and colleagues conducted in New Zealand ${ }^{255}$ may also be generalisable to Indigenous populations.

Implications of the recommendations. Laparoscopic ovarian surgery should be considered a second-line treatment indicated for infertile, anovulatory women with CCR PCOS. Laparoscopic ovarian surgery, when compared with other second-line treatments, is equally effective as gonadotrophins, with fewer multiple pregnancies. However, laparoscopic surgery, especially in overweight women, is associated with intra-operative risks (ie, difficulty with access to abdominal cavity and manipulation of surgical instruments, reduced operative field exposure) and postoperative risks (ie, bleeding, infection, thromboembolism, pulmonary atelectasis/ hypoxaemia, and wound complications). There is little research addressing the long-term complications of laparoscopic ovarian surgery. The guideline development group is not aware of changes to the way care is organised or barriers to implementation of these recommendations. Issues covered in the clinical practice point should be 
considered when deciding between laparoscopic ovarian surgery and gonadotrophins.

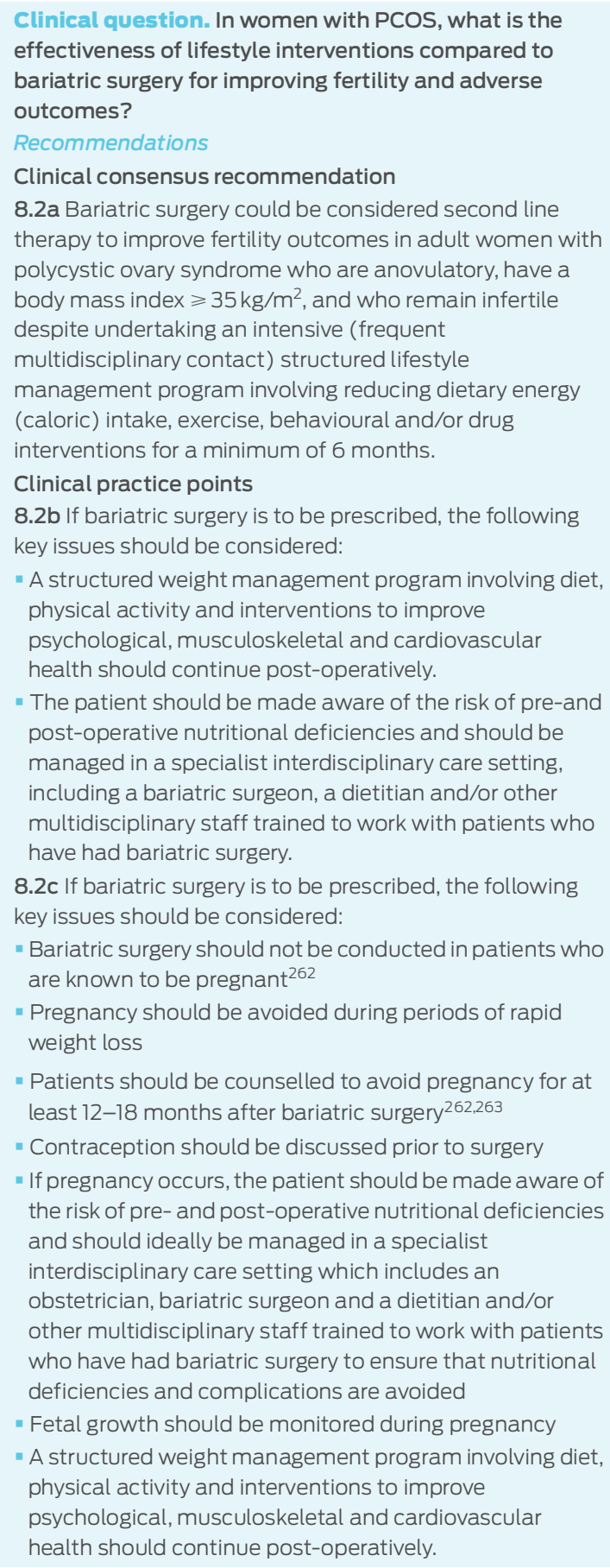

Context. Weight loss improves fertility and addresses maternal and neonatal complications associated with obesity, potentially also increasing the safety and effectiveness of ovulation induction. Intensive lifestyle intervention should be implemented for all overweight women wishing to improve fertility. It has been suggested that women with a BMI $\geqslant 35 \mathrm{~kg} / \mathrm{m}^{2}$ after failed attempts at weight loss for more than 1 year may consider bariatric surgery; however, surgical studies are generally poorly designed, ${ }^{264}$ and the 2009 American College of Obstetrics and Gynecology clinical practice guidelines do not currently recommend bariatric surgery for fertility treatment. $^{265}$

A recent meta-analysis of bariatric surgery in general populations reports weight loss of $38.5 \mathrm{~kg}$ or $56 \%$ excess weight loss and resolution of DM2 in $78 \%$ of patients with DM2. ${ }^{266}$ General international obesity guidelines ${ }^{267}$ recommended consideration of bariatric surgery with a $\mathrm{BMI} \geqslant 35 \mathrm{~kg} / \mathrm{m}^{2}$ and one or more complications, which are expected to abate with weight reduction, after failure of a structured lifestyle intervention. Surgery can be considered after unsuccessful lifestyle intervention with a BMI $\geqslant 40 \mathrm{~kg} / \mathrm{m}^{2}$ and can be first line with BMI $\geqslant 50 \mathrm{~kg} / \mathrm{m}^{2} .268$

Bariatric surgery has significant complications, including malabsorptive states, psychological issues, poor nutritional status, and disordered eating. Eating disorders are already relatively common among women with PCOS. Also, nutritional impact in pregnancy is unclear and although supplement use is widely recommended following bariatric surgery and for pregnant women, poor compliance is a problem. ${ }^{269}$

Specifically with regards to PCOS, recent guidelines from the American Association of Clinical Endocrinologists and the Obesity Society and the American Society for Metabolic and Bariatric Surgery noted a lack of conclusive clinical PCOS-specific evidence and suggested they should be advised that fertility status may be improved postoperatively. ${ }^{270}$

Overall, among people with morbid obesity, lifestyle interventions have very limited efficacy, but given the substantial efficacy of bariatric surgery for women who are severely obese, including in women with PCOS, it is important to determine the impact of bariatric surgery on infertility in women with PCOS. Benefits need to be balanced with risks of bariatric surgery, and controversies, including whether PCOS is considered an obesity-related condition and may reduce BMI cut-offs, ${ }^{271}$ need to be addressed. ${ }^{270}$ In this context, these issues were prioritised during guideline development.

Evidence informing the recommendations. We did not identify any PCOS-specific evidence to inform this recommendation and a clinical consensus recommendation has been made using the clinical expertise of the multidisciplinary guideline development group, informed by existing evidence-based clinical guidelines for bariatric surgery in the general population. In general populations, bariatric surgery is recommended for individuals with a $\mathrm{BMI} \geqslant 35 \mathrm{~kg} / \mathrm{m}^{2}$ and the presence of comorbid conditions that are expected to improve significantly with weight reduction. ${ }^{267,270}$ PCOS is a comorbid condition that improves significantly with weight reduction and is strongly associated with other comorbidities including DM2 and increased CVD risk. Therefore, it was concluded that general population evidence-based recommendations for bariatric surgery are applicable to this evidence review and patient population.

Implications of the recommendations. Bariatric surgery could be considered second-line therapy to improve fertility outcomes in PCOS. Current practice is unclear, although few women with PCOS are likely to be having this surgery. Implementation of the recommendations has resource implications including the cost of surgery and 
specialist care, potentially offset by less use of assisted reproductive technologies and fewer complications in pregnancy and beyond. Ethnic differences in obesity and complications are recognised and cut-off points for surgery may need to be adjusted for those at high risk. It is important to note that women with PCOS who become pregnant after bariatric surgery could be considered to have high-risk pregnancies, as are women in the general population who are obese and pregnant.

Research recommendation

Further methodologically rigorous trials are important to address the efficacy, safety and role of bariatric surgery in polycystic ovary syndrome including improving fertility. 


\section{Limitations of the guideline}

Given the complexity of PCOS, it was beyond the resources, time frames and scope of this guideline to address all aspects of this condition and the questions addressed here reflect a development and prioritisation process described in the full guideline. ${ }^{8}$ Generally in PCOS, there is a paucity of evidence and the evidence that is available is of poor quality; hence, consensus recommendations informed by evidence in other similar conditions often underpinned recommendations in this guideline. This guideline does not seek to provide full safety and usage information on pharmacological and surgical interventions. The pharmacological and surgical interventions recommended in the guideline should not be applied without consideration to the patient's clinical profile and personal preferences. It is recommended that the reader consults the full guideline, ${ }^{8}$ the Therapeutic Guidelines (http://www.tg.com.au) and the National
Prescribing Service (http://www.nps.org.au) for detailed prescribing information.

A formal analysis of cost-effectiveness was not completed. Consideration was given to cost in guideline development group meetings and impacted on recommendations. The guideline is based on the best evidence available up to November 2010. Development of technology and pharmacological intervention progresses rapidly. To ensure the guideline is updated appropriately, new significant publications will be inserted promptly if they have bearing on safety or if changes occur in the TGA registration status.

All recommendations are limited by their applicability and generalisability, therefore clinical judgement remains important. Finally, a guideline is only useful if it has been translated and implemented into practice appropriately. In doing so, cultural barriers may be experienced.

\begin{abstract}
Acknowledgements: We gratefully acknowledge the major contribution made by the Project Manager for the PCOS Australian Alliance and evidence-based guidelines, Linda Downes, Jean Hailes Foundation for Women's Health. We also acknowledge members of the Alliance who volunteered their time to aid the development of the guideline, including the Alliance Strategic Advisory Group (for membership, see Appendix II of full guideline ${ }^{8}$ ), the Jean Hailes Foundation for Women's Health team who contributed so much to this project over the past 2 years, the Australian Government Minister for Health and Ageing, the Hon Nicola Roxon MP, and the Australian Government Department of Health and Ageing for their support and funding of this guideline and the subsequent translation program. Finally, we acknowledge the commitment and support of the Centre for Clinical Effectiveness for providing evidence synthesis and guideline development expertise and to the Australasian Cochrane Centre for appraising the guidelines (using AGREE [Appraisal of Guidelines for Research and Evaluation] II).
\end{abstract}

Competing interests: Rob Norman has part-ownership in Fertility SA, a company providing fertility and IVF services in Adelaide; has received speaker fees from MSD Australia and Merck Serono Australia, received honoraria from MSD-Schering-Plough and Merck Serono Australia; and is a member of the MSD-Schering-Plough Advisory Board. Bronwyn Stuckey has received travel funding from Bayer Australia and ScheringPlough, both of which have interests in the oral contraceptives Diane-35 and Yasmin which are commonly prescribed to women with PCOS. Michael Costello has shares in IVF Australia; was the recipient of a grant from Schering-Plough in 2002 for research project unrelated to PCOS (Schering-Plough manufactures FSH injections for ovulation induction and ovarian stimulation); received sponsorship to attend and present at national and international scientific meetings on a broad range of topics determined by the individual conference organising committees, from pharmaceutical companies (Merck Serono Australia and Schering-Plough) who have a commercial interest in PCOS treatment products or guidelines; and is a member of the MSD-Schering-Plough Advisory Board. 
1 March W, Moore V, Willson K, et al. The prevalence of polycystic ovary syndrome in a community sample assessed under contrasting diagnostic criteria. Hum Reprod 2010; 25: 544-551.

2 Moran L, Teede H. Metabolic features of the reproductive phenotypes of polycystic ovary syndrome. Hum Reprod Update 2009; 15: 477-488.

3 Norman RJ, Dewailly D, Legro RS, Hickey TE. Polycystic ovary syndrome. Lancet 2007; 370: 685-697.

4 Ching H, Burke V, Stuckey B. Quality of life and psychological morbidity in women with polycystic ovary syndrome: body mass index, age and the provision of patient information are significant modifiers. Clin Endocrinol (Oxf) 2007; 66: 373-379.

5 Deeks A, Gibson-Helm M, Teede H. Anxiety and depression in polycystic ovary syndrome: a comprehensive investigation. Fertil Steril 2010; 93: 2421-2423.

6 Moran L, Pasquali R, Teede H, et al. Treatment of obesity in polycystic ovary syndrome: a position statement of the Androgen Excess and Polycystic Ovary Syndrome Society. Fertil Steril 2009; 92: 1966-1982.

7 Cussons A, Stuckey B, Walsh J, et al. Polycystic ovarian syndrome: marked differences between endocrinologists and gynaecologists in diagnosis and management. Clin Endocrinol (Oxf) 2005; 62: 289-295.

8 Evidence-based guideline for the assessment and management of polycystic ovary syndrome. Melbourne: Jean Hailes Foundation for Women's Health on behalf of the PCOS Australian Alliance, 2011.

9 Teede H, Deeks A, Moran L. Polycystic ovary syndrome: a complex condition with psychological, reproductive and metabolic manifestations that impacts on health across the lifespan. BMC Med 2010; 8: 41.

10 Moran L, Gibson-Helm M, Teede H, Deeks A. Polycystic ovary syndrome: a biopsychosocial understanding in young women to improve knowledge and treatment options. J Psychosom Obstet Gynaecol 2010; 31: 24-31.

11 Moran LJ, Misso ML, Wild RA, Norman RJ. Impaired glucose tolerance, type 2 diabetes and metabolic syndrome in polycystic ovary syndrome: a systematic review and meta-analysis. Hum Reprod Update 2010; 16: 347-363.

12 Boyle J. Polycystic ovary syndrome and associated metabolic features in Indigenous women in the Northern Territory [PhD thesis]. Adelaide: University of Adelaide, 2011.

13 Teede H, Zoungas S, Deeks A, et al. Check: Independent learning program for GPs. Polycystic ovary syndrome. Melbourne: Royal Australian College of General Practitioners, 2008.

14 Rotterdam ESHRE/ASRM-Sponsored PCOS Consensus Workshop Group. Revised 2003 consensus on diagnostic criteria and long-term health risks related to polycystic ovary syndrome. Fertil Steril 2004; 81: 19-25.

15 Zawadaki R, Dockerty M. Diagnostic criteria for polycystic ovarian syndrome: towards a rational approach. In: Dunaif A, Given JR, Haseltine F, Merriam GR, editors. Current issues in endocrinology and metabolism: polycystic ovary syndrome. Boston: Blackwell Scientific, 1992: 377-384.

16 Kristensen S, Ramlau-Hansen C, Ernst E, et al. A very large proportion of young Danish women have polycystic ovaries: is a revision of the Rotterdam criteria needed? Hum Reprod 2010; 25: 3117-3122.

17 Legro R, Strauss J. Molecular progress in infertility: polycystic ovary syndrome. Fertil Steril 2002; 78: 569-576.

18 Doi S, Al-Zaid M, Towers P, et al. Ovarian steroids modulate neuroendocrine dysfunction in polycystic ovary syndrome. J Endocrinol Invest 2005; 28: 882892.

19 Legro R, Castracane V, Kauffman R. Detecting insulin resistance in polycystic ovary syndrome: purposes and pitfalls. Obstet Gynecol Surv 2004; 59: 141-154.

20 Balen A, Conway G, Kaltsas G, et al. Polycystic ovary syndrome: the spectrum of the disorder in 1741 patients. Hum Reprod 1995; 10: 2107-2111.

21 Kiddy D, Sharp P, White D, et al. Differences in clinical and endocrine features between obese and non-obese subjects with polycystic ovary syndrome: an analysis of 263 consecutive cases. Clin Endocrinol (Oxf) 1990; 32: 213-220.

22 Shaw L, Bairey MerzC, Azziz R, et al. Postmenopausal women with a history of irregular menses and elevated androgen measurements at high risk for worsening cardiovascular event-free survival: results from the National Institutes of Health - National Heart, Lung, and Blood Institute sponsored Women's Ischemia Syndrome Evaluation. J Clin Endocrinol Metab 2008; 93 $1276-1284$.

23 Himelein M, Thatcher S. Polycystic ovary syndrome and mental health: a review. Obstet Gynecol Surv 2006; 61: 723-732.

24 Boomsma C, Eijkkemans M, Hughes E, et al. A meta-analysis of pregnancy outcomes in women with polycystic ovary syndrome. Hum Reprod Update 2006; 12:673-683.

25 Coffey $\mathrm{S}$, Mason $\mathrm{H}$. The effect of polycystic ovary syndrome on health-related quality of life. Gynecol Endocrinol 2003; 17: 379-386.

26 Haffner S. The insulin resistance syndrome revisited. Diabetes Care 1996; 19: 275.

27 Koskinen P, Manttari M, Manninen V. Coronary heart disease incidence in NIDDM patients in the Helsinki Heart study. Diabetes Care 1992; 15: 820.
28 Apridonidze T, Essah PA, luorno MJ, Nestler JE. Prevalence and characteristics of the metabolic syndrome in women with polycystic ovary syndrome. J Clin Endocrinol Metab 2005; 90: 1929-1935.

29 Meyer C, McGrath B, Cameron J, et al. Vascular dysfunction and metabolic parameters in polycystic ovary syndrome. J Clin Endocrinol Metab 2005; 90 : 4630-4635.

30 Meyer C, McGrath B, Teede H. Overweight women with polycystic ovary syndrome have evidence of subclinical cardiovascular disease. J Clin Endocrinol Metab 2005; 90: 5711-5716.

31 Women's Health Australia. The Australian Longitudinal Study on Women's Health. Australian women and their weight - a growing problem. Newcastle: WHA, 2005. http://www.alswh.org.au/Reports/Achievements/ achievements-weight.pdf (accessed Nov 2010).

32 Australian Institute of Health and Welfare. Australia's health 2006: the tenth biannual health report of the Australian Institute of Health and Welfare. Canberra: AlHW, 2006. (AlHW Cat. No. AUS 73.)

33 Finkelstein E, Fiebelkorn I, Wang G. National medical spending attributable to overweight and obesity: how much and who's paying? Health Aff (Millwood) 2003; Suppl Web Exclusives: W3-219-W3-226.

34 Wolf AM. Economic outcomes of the obese patient. Obes Res 2002; 10 Suppl 1: 58S-62S.

35 Hutchison S, Zoungas S, Teede $\mathrm{H}$. Insulin levels, insulin resistance and the use of metformin in polycystic ovary syndrome [letter]. Med J Aust 2007; 186: 268-269.

36 American Psychiatric Association. Diagnostic and statistical manual of mental disorders. 4th ed. Text revision. Arlington, Va: American Psychiatric Publishing Inc, 2000.

37 Australian Medical Association. Body image and health - 2002. Revised 2009 [position statement]. Canberra: AMA, 2009.

38 beyondblue. Clinical practice guidelines: depression in adolescents and young adults. Melbourne: beyondblue, 2011. http://www.beyondblue.org.au/ index.aspx?link_id=6.1247 (accessed Aug 2011).

39 National Collaborating Centre for Mental Health. Obsessive-compulsive disorder: core interventions in the treatment of obsessive-compulsive disorder and body dysmorphic disorder. London: National Institute for Health and Clinical Excellence, British Psychological Society, Royal College of Psychiatrists, 2006. (National Clinical Practice Guideline No. 31.)

40 National Institute for Health and Clinical Excellence. Depression in adults with a chronic physical health problem: treatment and management. London: National Collaborating Centre for Mental Health, National Health Service, 2009.

41 National Institute for Clinical Excellence. Eating disorders: core interventions in the treatment and management of anorexia nervosa, bulimia nervosa and related eating disorders. London: NICE, 2004.

42 National Institute for Health and Clinical Excellence. Depression: the treatment and management of depression in adults. London: National Collaborating Centre for Mental Health, National Health Service, 2009.

43 National Institute for Health and Clinical Excellence. Management of generalised anxiety disorder and panic disorder (with or without agoraphobia) in adults: management in primary, secondary and community care. London: National Collaborating Centre for Mental Health, National Collaborating Centre for Primary Care, National Health Service, 2011.

44 Martin KA, Chang RJ, Ehrmann DA, et al. Evaluation and treatment of hirsutism in premenopausal women: an Endocrine Society clinical practice guideline. J Clin Endocrinol Metab 2008; 93: 1105-1120.

45 National Health and Medical Research Council. National evidence based guidelines for the management of type 2 diabetes mellitus. Canberra: NHMRC, 2001.

46 National Vascular Disease Prevention Alliance. Guidelines for the assessment of absolute cardiovascular disease risk. Canberra: National Heart Foundation of Australia, 2009.

47 Meyer C, McGrath B, Teede H. Effects of medical therapy on insulin resistance and the cardiovascular system in polycystic ovary syndrome. Diabetes Care 2007; 30: 471-478.

48 Knowler W, Barrett-Connor E, Fowler S, et al. Reduction in the incidence of type 2 diabetes with lifestyle intervention or metformin. N Engl J Med 2002; 346: 393-403.

49 Teede $\mathrm{H}$, Hutchison S, Zoungas S. The management of insulin resistance in polycystic ovary syndrome. Trends Endocrinol Metab 2007; 18: 273-279.

50 Davis S, Knight S, White V, et al. Preliminary indication of a high prevalence of polycystic ovary syndrome in Indigenous Australian women. Gynecol Endocrinol 2002; 16: 443-446.

51 Australian Bureau of Statistics. National and Torres Strait Islander health survey 2004-05. Canberra: ABS, 2005. (ABS Cat. No. 4715.0.)

52 Vos T, Barker B, Stanley L, Lopez AD. The burden of disease and injury in Aboriginal and Torres Strait Islander peoples 2003. Brisbane: Centre for 
Burden of Disease and Cost-Effectiveness, School of Population Health, University of Queensland, 2007.

53 Azziz R, Carmina E, Dewailly E, et al. The Androgen Excess and PCOS Society criteria for the polycystic ovary syndrome: the complete task force report. Fertil Steril 2009; 91: 456-457.

54 National Health and Medical Research Council. Clinical practice guidelines for the management of overweight and obesity in adults. Canberra: Australian Government Publishing Service, 2004. http://www.health.gov.au/internet/ main/publishing.nsf/Content/obesityguidelines-guidelines-adults.htm (accessed Aug 2011).

55 National Health and Medical Research Council. NHMRC standards and procedures for externally developed guidelines. Canberra: NHMRC, 2007.

56 National Health and Medical Research Council. NHMRC levels of evidence and grades for recommendations for developers of guidelines. Canberra: NHMRC, 2009.

57 Southern Health Centre for Clinical Effectiveness. Critical appraisal templates. Melbourne: Southern Health, 2010.

58 Davison S, Bell R, Donath S, et al. Androgen levels in adult females: changes with age, menopause, and oophorectomy. J Clin Endocrinol Metab 2005; 90 : 3847-3853.

59 Vermeulen A, Verdonck L, Kaufman J. A critical evaluation of simple methods for the estimation of free testosterone in serum. J Clin Endocrinol Metab 1999 84: 3666-3672.

60 Escobar-Morreale HF, Asunción M, Calvo RM, et al. Receiver operating characteristic analysis of the performance of basal serum hormone profiles for the diagnosis of polycystic ovary syndrome in epidemiological studies. Eur JEndocrinol 2001; 145: 619-624.

61 Hahn S, Kuehnel W, Tan S, et al. Diagnostic value of calculated testosterone indices in the assessment of polycystic ovary syndrome. Clin Chem Lab Med 2007; 45: 202-207.

62 Koskinen P, Penttilä TA, Anttila L, et al. Optimal use of hormone determinations in the biochemical diagnosis of the polycystic ovary syndrome. Fertil Steril 1996; 65: 517-522.

63 Lemarchand-Béraud T, Zufferey M, Reymond M, Rey I. Maturation of the hypothalamo-pituitary-ovarian axis in adolescent girls. J Clin Endocrinol Metab 1982; 54: 241-246.

64 Treloar AE, Boynton RE, Behn BG, Brown BW. Variation of the human menstrual cycle through reproductive life. Int J Fertil 1967; 12: 77-126.

65 Flug D, Largo R, Prader A. Menstrual patterns in adolescent Swiss girls: a longitudinal study. Ann Hum Biol 1984; 11: 495-508.

66 Widholm 0 , Kantero R. A statistical analysis of the menstrual patterns of 8,000 Finnish girls and their mothers. Acta Obstet Gynecol Scand Suppl 1971; 14 Suppl 14: 1-36.

67 Adams Hillard P. Menstruation in young girls: a clinical perspective. Obstet Gynecol 2002; 99: 655-662.

68 Slap G. Menstrual disorders in adolescence. Best Pract Res Clin Obstet Gynaecol 2003; 17: 75-92.

69 Apter D, Vihko R. Early menarche, a risk factor for breast cancer, indicates early onset of ovulatory cycles. J Clin Endocrinol Metab 1983; 57: 82-86.

70 Hart R, Sloboda D, Doherty D, et al. Prenatal determinants of uterine volume and ovarian reserve in adolescence. J Clin Endocrinol Metab 2009; 94: 49314937.

71 Blank SK, Helm KD, McCartney CR, Marshall JC. Polycystic ovary syndrome in adolescence. Ann N Y Acad Sci 2008; 1135: 76-84.

72 Mortensen M, Rosenfield R, Littlejohn E. Functional significance of polycysticsize ovaries in healthy adolescents. J Clin Endocrinol Metab 2006; 91: 37863790.

73 Chen Y, Yang D, Li L, Chen X. The role of ovarian volume as a diagnostic criterion for Chinese adolescents with polycystic ovary syndrome. J Pediatr Adolesc Gynecol 2008; 21: 347-350.

74 San Martín-Rodríguez L, Beaulieu MD, D’Amour D, Ferrada-Videla M. The determinants of successful collaboration: a review of theoretical and empirical studies. J Interprof Care 2005; 19 Suppl 1: 132-147.

75 Smith G, Clarke D. Assessing the effectiveness of integrated interventions: terminology and approach. Med Clin North Am 2006; 90: 533-548.

76 Smith S, Allwright S, O'Dowd T. Effectiveness of shared care across the interface between primary and specialty care in chronic disease management. Cochrane Database Syst Rev 2007; (3): CD004910.

77 National Heart Foundation of Australia; Cardiac Society of Australia and New Zealand. Lipid management guidelines 2001 - summary paper. Med J Aust 2001; 175 (9 Suppl): S57-S88.

78 Wild R, Carmina E, Diamanti-Kandarakis E, et al. Assessment of cardiovascular risk and prevention of cardiovascular disease in women with the polycystic ovary syndrome: a consensus statement by the Androgen Excess and Polycystic Ovary Syndrome (AE-PCOS) Society. J Clin Endocrinol Metab 2010; 95: 2038-2049.
79 Tonkin A, Barter P, Best J, et al; National Heart Foundation of Australia; Cardiac Society of Australia and New Zealand. National Heart Foundation of Australia and the Cardiac Society of Australia and New Zealand: position statement of lipid management. Heart Lung Circ 2005; 14: 275-291.

80 National Blood Pressure and Vascular Disease Advisory Committee. Guide to management of hypertension 2008. Updated 2010. Canberra: National Heart Foundation of Australia, 2010.

81 Cussons A, Watts G, Burke V, et al. Cardiometabolic risk in polycystic ovary syndrome: a comparison of different approaches to defining the metabolic syndrome. Hum Reprod 2008; 23: 2352-2358.

82 Chan D, Watts G. Dyslipidaemia in the metabolic syndrome and type 2 diabetes: pathogenesis, priorities, pharmacotherapies. Expert Opin Pharmacother 2011; 12: 13-30.

83 Moran L, Hutchison S, Meyer C, et al. A comprehensive assessment of endothelial function in overweight women with and without polycystic ovary syndrome. Clin Sci (Lond) 2009; 116: 761-770.

84 Ehrmann D, Liljenquist D, Kasza K, et al. Prevalence and predictors of the metabolic syndrome in women with polycystic ovary syndrome. $J$ Clin Endocrinol Metab 2006; 91: 48-53.

85 Legro RS, Kunselman AR, Dodson WC, Dunaif A. Prevalence and predictors of risk for type 2 diabetes mellitus and impaired glucose tolerance in polycystic ovary syndrome: a prospective, controlled study in 254 affected women. JClin Endocrinol Metab 1999; 84: 165-168.

86 Legro R, Kunselman A, Dunaif A. Prevalence and predictors of dyslipidemia in women with polycystic ovary syndrome. Am J Med 2001; 111: 607-613.

87 Birdsall M, Farquhar C, White H. Association between polycystic ovaries and extent of coronary artery disease in women having cardiac catheterization. Ann Intern Med 1997; 126: 32-35.

88 Talbott E, Guzick D, Sutton-Tyrrell K, et al. Evidence for the association between polycystic ovary syndrome and premature carotid atherosclerosis in middle-aged women. Arterioscler Thromb Vasc Biol 2000; 20: 2414-2421.

89 Wild S, Pierpoint T, McKeigue P, Jacobs H. Cardiovascular disease in women with polycystic ovary syndrome at long-term follow-up: a retrospective cohort study. Clin Endocrinol (Oxf) 2000; 52: 595-600.

90 Solomon C. The epidemiology of polycystic ovary syndrome. Prevalence and associated disease risks. Endocrinol Metab Clin North Am 1999; 28: 247-263.

91 Gorgels W, vd Graaf Y, Blankenstein M, et al. Urinary sex hormone excretions in premenopausal women and coronary heart disease risk: a nested casereferent study in the DOM-cohort. J Clin Epidemiol 1997; 50: 275-281.

92 Pierpoint T, McKeigue $P$, Isaacs A, et al. Mortality of women with polycystic ovary syndrome at long term follow-up. J Clin Epidemiol 1998; 51: 581-586.

93 Rachon D, Teede H. Ovarian function and obesity - interrelationship, impact on women's reproductive lifespan and treatment options. Mol Cell Endocrinol 2010; 316: 172-179.

94 Australian Bureau of Statistics. Causes of death. Canberra: ABS, 2010. (ABS Cat. No. 3303.0.) http://abs.gov.au/AUSSTATS/abs@.nsf/allprimarymain features/5CCE256209F55B25CA2578840012A040?opendocument (accessed Aug 2011).

95 World Health Organization. Preventing chronic diseases: a vital investment: WHO global report. Geneva: WHO, 2005.

96 Geiss L, Herman W, Smith P. Mortality in non-insulin-dependent diabetes. In: Harris MI, Cowie CC, Stern MP, et al; National Diabetes Data Group, editors. Diabetes in America. 2nd ed. Washington, DC: US Government Printing Office, 1995: 233-257.

97 Chen L, Magliano D, Balkau B, et al. AUSDRISK: an Australian Type 2 Diabetes Risk Assessment Tool based on demographic, lifestyle and simple anthropometric measures. Med J Aust 2010; 192: 197-202.

98 Colagiuri S, Davies D, Girgis S, et al. National evidence based guideline for case detection and diagnosis of type 2 diabetes. Canberra: Diabetes Australia, NHMRC, 2009

99 Teede H, Stuckey B. Polycystic ovary syndrome and abnormal glucose tolerance [editorial]. Med J Aust 2007; 187: 324-325.

100 Reaven $G$. The insulin resistance syndrome: definition and dietary approaches to treatment. Annu Rev Nutr 2005; 25: 391-406.

101 DeUgarte C, Bartolucci C, Azziz R. Prevalence of insulin resistance in the polycystic ovary syndrome using the homeostasis model assessment. Fertil Steril 2005; 83: 1454-1460.

102 Acien P, Quereda F, Matallin P, et al. Insulin, androgens, and obesity in women with and without polycystic ovary syndrome: a heterogeneous group of disorders. Fertil Steril 1999; 72: 32-40.

103 Moran L, Strauss B, Teede H. Diabetes Risk Score in the diagnostic categories of polycystic ovary syndrome. Fertil Steril 2011; 95: 1742-1748.

104 Ehrmann D, Barnes R, Rosenfield R, et al. Prevalence of impaired glucose tolerance and diabetes in women with polycystic ovary syndrome. Diabetes Care 1999; 22: 141-146. 
105 Norman R, Masters L, Milner C, et al. Relative risk of conversion from normoglycaemia to impaired glucose tolerance or non-insulin dependent diabetes mellitus in polycystic ovarian syndrome. Hum Reprod 2001; 16: 19951998

106 Alberti K, Zimmet P, Shaw J. International Diabetes Federation: a consensus on type 2 diabetes prevention. Diabet Med 2007; 24: 451-463.

107 Azziz R, Carmina E, Dewailly D, et al. Position statement: criteria for defining polycystic ovary syndrome as a predominantly hyperandrogenic syndrome: an Androgen Excess Society guideline. J Clin Endocrinol Metab 2006; 91: 42374245.

108 Tomlinson J, Millward A, Stenhouse E, Pinkney J. Type 2 diabetes and cardiovascular disease in polycystic ovary syndrome: what are the risks and can they be reduced? Diabet Med 2010; 27: 498-515.

109 Bhattacharya S, Jha A. Prevalence and risk of depressive disorders in women with polycystic ovary syndrome (PCOS). Fertil Steril 2010; 94: 357-359.

110 Laggari V, Diareme S, Christogiorgos S, et al. Anxiety and depression in adolescents with polycystic ovary syndrome and Mayer-Rokitansky-KüsterHauser syndrome. J Psychosom Obstet Gynaecol 2009; 30: 83-88.

111 Australian Bureau of Statistics. ABS National Survey of Mental Health Wellbeing: summary of results, 2007. Canberra: ABS, 2008. (ABS Cat. No. 4326.0.)

112 Gwynn RC, McQuistion HL, McVeigh KH, et al. Prevalence, diagnosis, and treatment of depression and generalized anxiety disorder in a diverse urban community. Psychiatr Serv 2008; 59: 641-647.

113 Benson S, Hahn S, Tan S, et al. Prevalence and implications of anxiety in polycystic ovary syndrome: results of an internet-based survey in Germany. Hum Reprod 2009; 24: 1446-1451.

114 Deeks A, Gibson-Helm M, Teede H. Is having polycystic ovary syndrome (PCOS) a predictor of poor psychological function including depression and anxiety? Hum Reprod 2011; 26: 1399-1407.

115 Deeks A, Gibson-Helm M, Teede H. Negative body image and lower selfefficacy in women with polycystic ovary syndrome. Proceedings of the Australian Society for Behavioural Health and Medicine 8th Annual Scientific Conference; 2010; Feb 10-12; Brisbane.

116 Dawber R. Guidance for the management of hirsutism. Curr Med Res Opin 2005; 21: 1227-1234.

117 Trent M, Austin SB, Rich M, Gordon CM. Overweight status of adolescent girls with polycystic ovary syndrome: body mass index as mediator of quality of life. Ambul Pediatr 2005; 5: 107-111.

118 Hay P, Mond J, Buttner P, Darby A. Eating disorder behaviors are increasing: findings from two sequential community surveys in South Australia. PLoS One 2008; 3: el541.

119 Fairburn CG, Harrison PJ. Eating disorders. Lancet 2003; 361: 407-415.

120 McCluskey S, Lacey J, Pearce J. Binge-eating and polycystic ovaries. Lancet 1992; 340: 723.

121 Raphael F, Rodln D, Peattie A, et al. Ovarian morphology and insulin sensitivity in women with bulimia nervosa. Clin Endocrinol (Oxf) 1995; 43: 451455.

122 Hirschberg AL, Naessén S, Stridsberg M, et al. Impaired cholecystokinin secretion and disturbed appetite regulation in women with polycystic ovary syndrome. Gynecol Endocrinol 2004; 19: 79-87.

123 Jahanfar S, Eden J, Nguyent T. Bulimia nervosa and polycystic ovary syndrome. Gynecol Endocrinol 1995; 9: 113-117.

124 Månsson M, Holte J, Landin-Wilhelmsen K, et al. Women with polycystic ovary syndrome are often depressed or anxious - a case control study. Psychoneuroendocrinology 2008; 33: 1132-1138.

125 Fairburn CG, Wilson G, editors. Binge eating: nature, assessment and treatment. New York: Guilford, 1993.

126 Hay PP, Bacaltchuk J, Stefano S, Kashyap P. Psychological treatments for bulimia nervosa and binging. Cochrane Database Syst Rev 2009; (4): CD000562.

127 Shapiro J, Berkman N, Brownley K, et al. Bulimia nervosa treatment: a systematic review of randomized controlled trials. Int J Eat Disord 2007; 40: 321-336.

128 Wilson G. Psychological treatment of eating disorders. Annu Rev Clin Psychol 2005; 1: 439-465.

129 Watson J, Davies T. ABC of mental health: psychosexual problems. BMJ 1997; 315: 239-242.

130 Elsenbruch S, Hahn S, Kowalsky D, et al. Quality of life, psychosocial wellbeing, and sexual satisfaction in women with polycystic ovary syndrome. J Clin Endocrinol Metab 2003; 88: 5801-5807.

131 Hahn S, Benson S, Elsenbruch S, et al. Metformin treatment of polycystic ovary syndrome improves health-related quality-of-life, emotional distress and sexuality. Hum Reprod 2006; 21: 1925-1934.
132 Hahn S, Janssen O, Tan S, et al. Clinical and psychological correlates of quality-of-life in polycystic ovary syndrome. Eur J Endocrinol 2005; 153: 853860

133 Janssen 0, Hahn S, Tan S, et al. Mood and sexual function in polycystic ovary syndrome. Semin Reprod Med 2008; 26: 45-52.

134 Drosdzol A, Skrzypulec V, Mazur B, Pawliñska-Chmara R. Quality of life and marital sexual satisfaction in women with polycystic ovary syndrome. Folia Histochem Cytobiol 2007; 45 Suppl 1: S93-S97.

135 GlueckC, Dharashivkar S, Wang P, et al. Obesity and extreme obesity, manifest by ages 20-24 years, continuing through 32-41 years in women, should alert physicians to the diagnostic likelihood of polycystic ovary syndrome as a reversible underlying endocrinopathy. Eur J Obstet Gynecol Reprod Biol 2005; 122: 206-212.

136 Teede H, Deeks A, Gibson-Helm M, et al. Body mass index as a predictor of polycystic ovary syndrome risk: results of a longitudinal cohort study [abstract no. 246]. Endocrine Society Annual Meeting; 2010 Jun 19-22; San Diego, Calif.

137 Clark AM, Thornley B, Tomlinson L, et al. Weight loss in obese infertile women results in improvement in reproductive outcome for all forms of fertility treatment. Hum Reprod 1998; 13: 1502-1505.

138 Huber-Buchholz M, Carey D, Norman R. Restoration of reproductive potential by lifestyle modification in obese polycystic ovary syndrome: role of insulin sensitivity and luteinizing hormone. J Clin Endocrinol Metab 1999; 84: 14701474

139 Moran $\sqcup$, Noakes M, Clifton PM, et al. Dietary composition in restoring reproductive and metabolic physiology in overweight women with polycystic ovary syndrome. J Clin Endocrinol Metab 2003; 88: 812-819.

140 Thomson RL, Buckley JD, Lim SS, et al. Lifestyle management improves quality of life and depression in overweight and obese women with polycystic ovary syndrome. Fertil Steril 2010; 94: 1812-1816.

141 Andersen P, Seljeflot I, Abdelnoor M, et al. Increased insulin sensitivity and fibrinolytic capacity after dietary intervention in obese women with polycystic ovary syndrome. Metabolism 1995; 44: 611-616.

142 Clark AM, Ledger W, Galletly C, et al. Weight loss results in significant improvement in pregnancy and ovulation rates in anovulatory obese women. Hum Reprod 1995; 10: 2705-2712.

143 Crave JC, Fimbel S, Lejeune H, et al. Effects of diet and metformin administration on sex hormone-binding globulin, androgens, and insulin in hirsute and obese women. J Clin Endocrinol Metab 1995; 80: 2057-2062.

144 Crosignani PG, Colombo M, Vegetti W, et al. Overweight and obese anovulatory patients with polycystic ovaries: parallel improvements in anthropometric indices, ovarian physiology and fertility rate induced by diet. Hum Reprod 2003; 18: 1928-1932.

145 Gambineri A, Pelusi C, Genghini S, et al. Effect of flutamide and metformin administered alone or in combination in dieting obese women with polycystic ovary syndrome. Clin Endocrinol (Oxf) 2004; 60: 241-249.

146 Guzick DS, Wing R, Smith D, et al. Endocrine consequences of weight loss in obese, hyperandrogenic, anovulatory women. Fertil Steril 1994; 61: 598-604.

147 Holte J, Bergh T, Berne C, et al. Restored insulin sensitivity but persistently increased early insulin secretion after weight loss in obese women with polycystic ovary syndrome. J Clin Endocrinol Metab 1995; 80: 2586-2593.

148 Jakubowicz DJ, Nestler JE. 17 alpha-hydroxyprogesterone responses to leuprolide and serum androgens in obese women with and without polycystic ovary syndrome offer dietary weight loss. J Clin Endocrinol Metab 1997; 82: 556-560.

149 Kiddy DS, Hamilton-Fairley D, Bush A, et al. Improvement in endocrine and ovarian function during dietary treatment of obese women with polycystic ovary syndrome. Clin Endocrinol (Oxf) 1992; 36: 105-111.

150 Kiddy DS, Hamilton-Fairley D, Seppälä M, et al. Diet-induced changes in sex hormone binding globulin and free testosterone in women with normal or polycystic ovaries: correlation with serum insulin and insulin-like growth factor-I. Clin Endocrinol (Oxf) 1989; 31: 757-763.

151 Moran $\sqcup$, Noakes M, Clifton PM, et al. C-reactive protein before and after weight loss in overweight women with and without polycystic ovary syndrome. J Clin Endocrinol Metab 2007; 92: 2944-2951.

152 Moran L, Noakes M, Clifton PM, et al. Short-term meal replacements followed by dietary macronutrient restriction enhance weight loss in polycystic ovary syndrome. Am J Clin Nutr 2006; 84: 77-87.

153 Pasquali R, Antenucci D, Casimirri F, et al. Clinical and hormonal characteristics of obese amenorrheic hyperandrogenic women before and after weight loss. J Clin Endocrinol Metab 1989; 68: 173-179.

154 Pasquali R, Gambineri A, Biscotti D, et al. Effect of long-term treatment with metformin added to hypocaloric diet on body composition, fat distribution, and androgen and insulin levels in abdominally obese women with and without the polycystic ovary syndrome. J Clin Endocrinol Metab 2000; 85: 2767-2774. 
155 Qublan HS, Yannakoula EK, Al-Qudah MA, El-Uri FI. Dietary intervention versus metformin to improve the reproductive outcome in women with polycystic ovary syndrome. A prospective comparative study. Saudi Med J 2007; 28: 1694-1699.

156 Stamets K, Taylor DS, Kunselman A, et al. A randomized trial of the effects of two types of short-term hypocaloric diets on weight loss in women with polycystic ovary syndrome. Fertil Steril 2004; 81: 630-637.

157 Tang T, Glanville J, Hayden CJ, et al. Combined lifestyle modification and metformin in obese patients with polycystic ovary syndrome. A randomized, placebo-controlled, double-blind multicentre study. Hum Reprod 2006; 21: 80-89.

158 Van Dam EW, Roelfsema F, Veldhuis JD, et al. Increase in daily LH secretion in response to short-term calorie restriction in obese women with PCOS. Am J Physiol Endocrinol Metab 2002; 282: E865-E872.

159 Wahrenberg $\mathrm{H}, \mathrm{Ek}$ I, Reynisdottir S, et al. Divergent effects of weight reduction and oral anticonception treatment on adrenergic lipolysis regulation in obese women with the polycystic ovary syndrome. J Clin Endocrinol Metab 1999; 84 2182-2187.

160 Hutchison SK, Stepto NK, Harrison CL, et al. Effects of exercise on insulin resistance and body composition in overweight and obese women with and without polycystic ovary syndrome. JClin Endocrinol Metab 2011; 96: E48-E56.

161 Poehlman ET, Dvorak RV, DeNino WF, et al. Effects of resistance training and endurance training on insulin sensitivity in nonobese, young women: a controlled randomized trial. J Clin Endocrinol Metab 2000; 85: 2463-2468.

162 Ross R, Dagnone D, Jones P, et al. Reduction in obesity and related comorbid conditions after diet-induced weight loss or exercise-induced weight loss in men. Ann Intern Med 2000; 133: 92-103.

163 Moran LJ, Hutchison SK, Norman RJ, Teede HJ. Lifestyle changes in women with polycystic ovary syndrome. Cochrane Database Syst Rev 2011; (2): CD007506.

164 Palomba S, Giallauria F, Falbo A, et al. Structured exercise training programme versus hypocaloric hyperproteic diet in obese polycystic ovary syndrome patients with anovulatory infertility: a 24-week pilot study. Hum Reprod 2008; 23: 642-650.

165 Hession M, Rolland C, Kulkarni U, et al. Systematic review of randomized controlled trials of low-carbohydrate vs. low-fat/low-calorie diets in the management of obesity and its comorbidities. Obes Rev 2009; 10: 36-50.

166 Pirozzo S, Summerbell C, Cameron C, Glaziou P. Advice on low-fat diets for obesity. Cochrane Database Syst Rev 2002; (2): CD003640.

167 Sacks FM, Bray GA, Carey VJ, et al. Comparison of weight-loss diets with different compositions of fat, protein, and carbohydrates. N Engl J Med 2009; 360: 859-873.

168 Galletly C, Moran L, Noakes M, et al. Psychological benefits of a high-protein, low-carbohydrate diet in obese women with polycystic ovary syndrome - a pilot study. Appetite 2007; 49: 590-593.

169 Douglas CC, Gower BA, Darnell BE, et al. Role of diet in the treatment of polycystic ovary syndrome. Fertil Steril 2006; 85: 679-688.

170 Marsh KA, Steinbeck KS, Atkinson FS, et al. Effect of a low glycemic index compared with a conventional healthy diet on polycystic ovary syndrome. Am JClin Nutr 2010; 92: 83-92.

171 Children's Health Development Foundation, South Australia; Deakin University. The Australian guide to healthy eating. Canberra: Australian Government Department of Health and Ageing, 1998.

172 Miller W, Rollnick S. Motivational interviewing: preparing people for change 2nd ed. New York: Guilford Press, 2002.

173 Avenell A, Broom J, Brown TJ, et al. Systematic review of the long-term effects and economic consequences of treatments for obesity and implications for health improvement. Health Technol Assess 2004; 8: iii-iv, 1-182.

174 Ogilvie D, Foster CE, Rothnie H, et al. Interventions to promote walking: systematic review. BMJ 2007; 334: 1204.

175 Dombrowski SU, Sniehotta FF, Avenell A, et al. Identifying active ingredients in complex behavioural interventions for obese adults with obesity-related comorbidities or additional risk factors for co-morbidities: a systematic review. Health Psychol Rev 2010. [Epub ahead of print]. doi: 10.1080/17437199.2010. 513298

176 Gillies CL, Abrams KR, Lambert PC, et al. Pharmacological and lifestyle interventions to prevent or delay type 2 diabetes in people with impaired glucose tolerance: systematic review and meta-analysis. BMJ 2007; 334: 299

177 Thompson RL, Summerbell CD, Hooper L, et al. Dietary advice given by a dietitian versus other health professional or self-help resources to reduce blood cholesterol. Cochrane Database Syst Rev 2003; (3): CD001366.

178 Shaw K, O'Rourke P, Del Mar C, Kenardy J. Psychological interventions for overweight or obesity. Cochrane Database Syst Rev 2005; (2): CD003818.

179 Kasim-Karakas S, Almario R, Cunningham W. Effects of protein versus simple sugar intake on weight loss in polycystic ovary syndrome (according to the National Institutes of Health criteria). Fertil Steril 2009; 92: 262-270.
180 Atiomo W, Read A, Golding M, et al. Local recruitment experience in a study comparing the effectiveness of a low glycaemic index diet with a low calorie healthy eating approach at achieving weight loss and reducing the risk of endometrial cancer in women with polycystic ovary syndrome (PCOS). Contemp Clin Trials 2009; 30: 451-456.

181 Pate RR, Pratt M, Blair SN, et al. Physical activity and public health: a recommendation from the Centers for Disease Control and Prevention and the American College of Sports Medicine. JAMA 1995; 273: 402-407.

182 Richter EA, Mikines KJ, Galbo H, Kiens B. Effect of exercise on insulin action in human skeletal muscle. J Appl Physiol 1989; 66: 876-885.

183 Tuomilehto J, Lindström J, Eriksson JG, et al. Prevention of type 2 diabetes mellitus by changes in lifestyle among subjects with impaired glucose tolerance. NEngl J Med 2001; 344: 1343-1350.

184 Goodyear $\amalg$, Kahn BB. Exercise, glucose transport, and insulin sensitivity. Annu Rev Med 1998; 49: 235-261.

185 Shephard RJ, Balady GJ. Exercise as cardiovascular therapy. Circulation 1999; 99: 963-972.

186 Cuff DJ, Meneilly GS, Martin A, et al. Effective exercise modality to reduce insulin resistance in women with type 2 diabetes. Diabetes Care 2003; 26 2977-2982.

187 Maiorana A, O'Driscoll G, Goodman C, et al. Combined aerobic and resistance exercise improves glycemic control and fitness in type 2 diabetes. Diabetes Res Clin Pract 2002; 56: 115-123.

188 Park SK, Park JH, Kwon YC, et al. The effect of combined aerobic and resistance exercise training on abdominal fat in obese middle-aged women. $J$ Physiol Anthropol Appl Human Sci 2003; 22: 129-135.

189 Sigal RJ, Kenny GP, Boulé NG, et al. Effects of aerobic training, resistance training, or both on glycemic control in type 2 diabetes: a randomized trial. Ann Intern Med 2007; 147: 357-369.

190 Ross R, Bradshaw AJ. The future of obesity reduction: beyond weight loss. Nat Rev Endocrinol 2009; 5: 319-325.

191 Orio F, Giallauria F, Palomba S, et al. Metabolic and cardiopulmonary effects of detraining after a structured exercise training programme in young PCOS women. Clin Endocrinol (Oxf) 2008; 68: 976-981.

192 Thomson RL, Buckley JD, Noakes M, et al. The effect of a hypocaloric diet with and without exercise training on body composition, cardiometabolic risk profile, and reproductive function in overweight and obese women with polycystic ovary syndrome. J Clin Endocrinol Metab 2008; 93: 3373-3380.

193 Brown AJ, Setji TL, Sanders LL, et al. Effects of exercise on lipoprotein particles in women with polycystic ovary syndrome. Med Sci SPorts Exerc 2009; 41: 497504.

194 Bruner B, Chad K, Chizen D. Effects of exercise and nutritional counseling in women with polycystic ovary syndrome. Appl Physiol Nutr Metab 2006; 31: 384-391.

195 Giallauria F, Palomba S, Maresca L, et al. Exercise training improves autonomic function and inflammatory pattern in women with polycystic ovary syndrome (PCOS). Clin Endocrinol (Oxf) 2008; 69: 792-798.

196 Stener-Victorin E, Jedel E, Janson PO, Sverrisdottir YB. Low-frequency electroacupuncture and physical exercise decrease high muscle sympathetic nerve activity in polycystic ovary syndrome. Am J Physiol Regul Integr Comp Physiol 2009; 297: R387-R395.

197 Vigorito C, Giallauria F, Palomba S, et al. Beneficial effects of a three-month structured exercise training program on the cardiopulmonary functional capacity in young women with polycystic ovary syndrome. J Clin Endocrinol Metab 2007: 92: 1379-1384.

198 American College of Sports Medicine; American Heart Association. Physical activity and public health guidelines. Indianapolis: ACSM, 2007. http:// www.acsm.org/AM/Template.cfm?Section=Home_Page\&TEMPLATE=/CM/ HTMLDisplay.cfm\&CONTENTID=7764 (accessed Aug 2011)

199 Briffa T, Maiorana A, Allan R, et al; Executive Working Group and National Forum Participants. National Heart Foundation of Australia physical activity recommendations for people with cardiovascular disease. Sydney: National Heart Foundation of Australia, 2006

200 Australian Government Department of Health and Ageing. National physical activity guidelines. Canberra: DoHA, 2010. http://www.health.gov.au/ internet/main/publishing.nsf/Content/health-pubhlth-strateg-phys-actguidelines (accessed Aug 2011).

201 Haskell WL, Lee IM, Pate RR, et al. Physical activity and public health: updated recommendation for adults from the American College of Sports Medicine and the American Heart Association. Circulation 2007; 116: 1081-1093.

202 Nelson ME, Rejeski WJ, Blair SN, et al. Physical activity and public health in older adults: recommendation from the American College of Sports Medicine and the American Heart Association. Circulation 2007; 116: 1094-1105.

203 Norton K, Norton L, Sadgrove D. Position statement on physical activity and exercise intensity terminology. J Sci Med Sport 2010; 13: 496-502. 
204 Physical Activity Guidelines Advisory Committee. Physical Activity Guidelines Advisory Committee report 2008. Washington, DC: Department of Health and Human Services, 2008.

205 World Health Organization. Global strategy on diet, physical activity and health. Geneva: WHO, 2004.

206 World Health Organization. A guide for population-based approaches to increasing levels of physical activity: implementation of the WHO global strategy on diet, physical activity and health. Geneva: WHO, 2007.

207 Harrison CL, Lombard CB, Moran LJ, Teede HJ. Exercise therapy in polycystic ovary syndrome: a systematic review. Hum Reprod Update 2011; 17: 171-183.

208 Tang T, Lord JMM, Norman R, et al. Insulin-sensitising drugs (metformin, rosiglitazone, pioglitazone, D-chiro-inositol) for women with polycystic ovary syndrome, oligo amenorrhoea and subfertility. Cochrane Database Syst Rev 2010; (1): CD003053.

209 Hoeger K, Davidson K, Kochman L, et al. The impact of metformin, oral contraceptives, and lifestyle modification on polycystic ovary syndrome in obese adolescent women in two randomized, placebo-controlled clinical trials. J Clin Endocrinol Metab 2008; 93: 4299-4306.

210 Palomba S, Falbo A, Giallauria F, et al. Six weeks of structured exercise training and hypocaloric diet increases the probability of ovulation after clomiphene citrate in overweight and obese patients with polycystic ovary syndrome: a randomized controlled trial. Hum Reprod 2010; 25: 2783-2791.

211 Gambineri A, Patton L, Vaccina A, et al. Treatment with flutamide, metformin, and their combination added to a hypocaloric diet in overweight-obese women with polycystic ovary syndrome: a randomized, 12-month, placebocontrolled study. J Clin Endocrinol Metab 2006; 91: 3970-3980.

212 Hoeger KM, Kochman L, Wixom N, et al. A randomized, 48-week, placebocontrolled trial of intensive lifestyle modification and/or metformin therapy in overweight women with polycystic ovary syndrome: a pilot study. Fertil Steril 2004; 82: 421-429.

213 Karimzadeh MA, Javedani M. An assessment of lifestyle modification versus medical treatment with clomiphene citrate, metformin, and clomiphene citrate-metformin in patients with polycystic ovary syndrome. Fertil Steril 2010; $94: 216-220$.

214 Otta CF, Wior M, Iraci GS, et al. Clinical, metabolic, and endocrine parameters in response to metformin and lifestyle intervention in women with polycystic ovary syndrome: a randomized, double-blind, and placebo control trial. Gynecol Endocrinol 2010; 26: 173-178.

215 Royal Australian and New Zealand College of Obstetricians and Gynaecologists. C-Gyn 2: ovarian stimulation in infertility. Melbourne: RANZCOG, 2008.

216 Shelly W, Draper MW, Krishnan V, et al. Selective estrogen receptor modulators: an update on recent clinical findings. Obstet Gynecol Surv 2008; 63: 163-181.

217 Adashi EY.Clomiphene citrate: mechanism(s) and site(s) of action - a hypothesis revisited. Fertil Steril 1984; 42: 331-344.

218 Palomba S, Falbo A, Zullo F. Management strategies for ovulation induction in women with polycystic ovary syndrome and known clomifene citrate resistance. Curr Opin Obstet Gynecol 2009; 21: 465-473.

219 Kafy S, Tulandi T. New advances in ovulation induction. Curr Opin Obstet Gynecol 2007; 19: 248-252.

220 Rossing MA, Daling JR, Weiss NS, et al. Ovarian tumours in a cohort of infertile women. N Engl J Med 1994; 331: 771-776.

221 Brown J, Farquhar C, Beck J, et al. Clomiphene and anti-oestrogens for ovulation induction in PCOS. Cochrane Database Syst Rev 2009; (4): CD002249.

222 Palomba S, Falbo A, Zullo F, Orio F Jr. Evidence-based and potential benefits of metformin in the polycystic ovary syndrome: a comprehensive review. Endocr Rev 2009; 30: 1-50.

223 Costello MF, Eden JA. A systematic review of the reproductive system effects of metformin in patients with polycystic ovary syndrome. Fertil Steril 2003; 79: 1-13.

224 Creanga AA, Bradley HM, McCormick C, Witkop CT. Use of metformin in polycystic ovary syndrome: a meta-analysis. Obstet Gynecol 2008; 111: 959968.

225 Moll E, van der Veen F, van Wely M. The role of metformin in polycystic ovary syndrome: a systematic review. Hum Reprod Update 2007; 13: 527-537.

226 Johnson NP, Stewart AW, Falkiner J, et al. PCOSMIC: a multi-centre randomized trial in women with polycystic ovary syndrome evaluating metformin for infertility with clomiphene. Hum Reprod 2010; 25: 1675-1683.

227 Kazerooni T, Ghaffarpasand F, Kazerooni Y, et al. Short-term metformin treatment for clomiphene citrate-resistant women with polycystic ovary syndrome. Int J Gynaecol Obstet 2009; 107: 50-53.

228 Siebert TI, Kruger TF, Lombard C. Evaluating the equivalence of clomiphene citrate with and without metformin in ovulation induction in PCOS patients. Assist Reprod Genet 2009; 26: 165-171.
229 Ben Ayed B, Dammak dit Mlik S, Ben Arab H, et al. Metformin effects on clomifene-induced ovulation in the polycystic ovary syndrome. Tunis Med 2009; 87: 43-49.

230 Palomba S, Pasquali R, Orio F Jr, Nestler JE. Clomiphene citrate, metformin or both as first-step approach in treating anovulatory infertility in patients with polycystic ovary syndrome (PCOS): a systematic review of head-to-head randomized controlled studies and meta-analysis. Clin Endocrinol (Oxf) 2009; 70: 311-321.

231 Nugent D, Vanderkerchove P, Hughes E, et al. Gonadotrophin therapy for ovulation induction in subfertility associated with polycystic ovary syndrome. Cochrane Database Syst Rev 2000; (3): CD000410.

232 Messinis IE. Ovulation induction: a mini review. Hum Reprod 2005; 20: 26882697.

233 Macklon N, Fauser B. The step-down protocol. In: Tarlatzis B, editor. Ovulation induction [European practice in obstetrics and gynaecology series]. Paris: Elsevier, 2002.

234 López E, Gunby J, Daya S, et al. Ovulation induction in women with polycystic ovary syndrome: randomized trial of clomiphene citrate versus low-dose recombinant FSH as first line therapy. Reprod Biomed Online 2004; 9: 382390.

235 Homburg R, Hendriks M, Konig T, et al. Clomifene or low dose FSH for the first line treatment of anovulatory PCOS: a prospective randomised multinational study (COFFI) [oral abstract 0-058]. Conference of the European Society of Human Reproduction and Embryology; 2009; Amsterdam.

236 Mitwally MF, Casper RF. Use of an aromatase inhibitor for induction of ovulation in patients with an inadequate response to clomiphene citrate. Fertil Steril 2001; 75: 305-309.

237 Holzer H, Casper R, Tulandi T. A new era in ovulation induction. Fertil Steril 2006; 85: 277-284

238 Healey S, Tan SL, Tulandi T, Biljan MM. Effects of letrozole on superovulation with gonadotrophins in women undergoing intrauterine insemination. Fertil Steril 2003; 80: 1325-1329.

239 Casper RF. Letrozole: ovulation or superovulation? Fertil Steril 2003; 80: 13351337.

240 Biljan M, Hemmings R, Brassard N. The outcome of 150 babies following the treatment with letrozole or letrozole and gonadotropins [abstract 1033]. Fertil Steril 2005; 84: 0-231.

241 Forman R, Gill S, Moretti M, et al. Fetal safety of letrozole and clomiphene citrate for ovulation induction. J Obstet Gynaecol Can 2007; 29: 668-671.

242 Tulandi T, Martin J, Al-Fadhli R, et al. Congenital malformations among 911 newborns conceived after infertility treatment with letrozole or clomiphene citrate. Fertil Steril 2006; 85: 1761-1765.

243 Kamath MS, Aleyamma TK, Chandy A, George K. Aromatase inhibitors in women with clomiphene citrate resistance: a randomized, double-blind, placebo-controlled trial. Fertil Steril 2010; 94: 2857-2859.

244 Al-Omari WR, Sulaiman WR, Al-Hadithi N. Comparison of two aromatase inhibitors in women with clomiphene-resistant polycystic ovary syndrome. Int J Gynaecol Obstet 2004; 85: 289-291.

245 Badawy A, Mosbah A, Shady M, et al. Anastrozole or letrozole for ovulation induction in clomiphene-resistant women with polycystic ovarian syndrome: a prospective randomized trial. Fertil Steril 2008; 89: 1209-1212.

246 Badawy A, Mosbah A, Tharwat A, et al. Extended letrozole therapy for ovulation induction in clomiphene-resistant women with polycystic ovary syndrome: a novel protocol. Fertil Steril 2009; 92: 236-239.

247 Bayar U, Kiran S, Coskun A, et al. Use of an aromatase inhibitor in patients with polycystic ovary syndrome: a prospective randomized trial. Fertil Steril 2006; 86: 1447-1451.

248 Badawy A, Abdel Aal I, Abulatta M, et al. Clomiphene citrate or letrozole for ovulation induction in women with polycystic ovarian syndrome: a prospective randomized trial. Fertil Steril 2009; 92: 849-852.

249 Dehbashi S, Kazerooni T, Robati M, et al. Comparison of the effects of letrozole and clomiphene citrate on ovulation and pregnancy rate in patients with polycystic ovary syndrome. Iran J Med Sci 2009; 34: 23-28.

250 Begum MR, Ferdous J, Begum A, Quadir E. Comparison of efficacy of aromatase inhibitor and clomiphene citrate in induction of ovulation in polycystic ovarian syndrome. Fertil Steril 2009; 92: 853-857.

251 Atay V,Cam C, Muhcu M, et al. Comparison of letrozole and clomiphene citrate in women with polycystic ovaries undergoing ovarian stimulation. J Int Med Res 2006; 34: 73-76.

252 Zeinalzadeh M, Basirat Z, Esmailpour M. Efficacy of letrozole in ovulation induction compared to that of clomiphene citrate in patients with polycystic ovarian syndrome. J Reprod Med 2010; 55: 36-40.

253 Abu Hashim H, Shokeir T, Badawy A. Letrozole versus combined metformin and clomiphene citrate for ovulation induction in clomiphene-resistant women with polycystic ovary syndrome: a randomized controlled trial. Fertil Steril 2010; 94: 1405-1409. 
254 Gjönnaess H. Polycystic ovarian syndrome treated by ovarian electrocautery through the laparoscope. Fertil Steril 1984; 41: 20-25.

255 Farquhar C, Lilford R, Marjoribanks J, Van dekerckhove P. Laparoscopic "drilling" by diathermy or laser for ovulation induction in anovulatory polycystic ovary syndrome. Cochrane Database Syst Rev 2007; (3): CD001122.

256 Palomba S, Falbo A, Battista L, et al. Laparoscopic ovarian diathermy vs clomiphene citrate plus metformin as second-line strategy for infertile anovulatory patients with polycystic ovary syndrome: a randomized controlled trial. Am J Obstet Gynecol 2010; 202: el-e8.

257 Hamed HO, Hasan AF, Ahmed OG, Ahmed MA. Metformin versus laparoscopic ovarian drilling in clomiphene- and insulin-resistant women with polycystic ovary syndrome. Int J Gynaecol Obstet 2010; 108: 143-147.

258 Palomba S, Orio F Jr, Nardo LG, et al. Metformin administration versus laparoscopic ovarian diathermy in clomiphene citrate-resistant women with polycystic ovary syndrome: a prospective parallel randomized double-blind placebo-controlled trial. J Clin Endocrinol Metab 2004; 89: 4801-4809.

259 Palomba S, Orio F Jr, Falbo A, et al. Plasminogen activator inhibitor 1 and miscarriage after metformin treatment and laparoscopic ovarian drilling in patients with polycystic ovary syndrome. Fertil Steril 2005; 84: 761-765.

260 Amer SA, Li TC, Metwally M, et al. Randomized controlled trial comparing laparoscopic ovarian diathermy with clomiphene citrate as a first-line method of ovulation induction in women with polycystic ovary syndrome. Hum Reprod 2009; 24: 219-225.

261 Farquhar C, Williamson K, Gudex G, et al. A randomized controlled trial of laparoscopic ovarian diathermy versus gonadotrophin therapy for women with clomiphene citrate-resistant polycystic ovary syndrome. Fertil Steril 2002; 78: 404-411.

262 Apovian CM, Cummings S, Anderson W, et al. Best practice updates for multidisciplinary care in weight loss surgery. Obesity (Silver Spring) 2009; 17: 871-879.
263 Heber D, Greenway FL, Kaplan LM, et al. Endocrine and nutritional management of the post-bariatric surgery patient: an endocrine society clinical practice guideline. J Clin Endocrinol Metab 2010; 95: 4823-4843.

264 Shah DK, Ginsburg ES. Bariatric surgery and fertility. Curr Opin Obstet Gynecol 2010; 22: 248-254.

265 American College of Obstetrics and Gynecology. Clinical management guidelines for obstetrician-gynecologists. Washington, DC: ACOG, 2009

266 Picot J, Jones J, Colquitt J, et al. The clinical effectiveness and costeffectiveness of bariatric (weight loss) surgery for obesity: a systematic review and economic evaluation. Health Technol Assess 2009; 13: 1-190, 215357, iii-iv.

267 Scottish Intercollegiate Guidelines Network. Management of obesity: a national clinical guideline. Edinburgh: SIGN, 2010.

268 National Institute for Health and Clinical Excellence. NICE clinical guideline 43. Obesity: guidance on the prevention, identification, assessment and management of overweight and obesity in adults and children. London, 2006.

269 Nilsen RM, Vollset SE, Gjessing HK, et al. Patterns and predictors of folic acid supplement use among pregnant women: the Norwegian Mother and Child Cohort Study. Am J Clin Nutr 2006; 84: 1134-1141.

270 Mechanick J, Kushner RF, Sugerman HJ, et al; American Association of Clinical Endocrinologists; Obesity Society; American Society for Metabolic \& Bariatric Surgery. American Association of Clinical Endocrinologists, The Obesity Society, and American Society for Metabolic \& Bariatric Surgery medical guidelines for clinical practice for the perioperative nutritional, metabolic, and nonsurgical support of the bariatric surgery patient. Obesity (Silver Spring) 2009; 17 Suppl 1: S1-S70, v.

271 Rubino F, Kaplan LM, Schauer PR, Cummings DE. The Diabetes Surgery Summit consensus conference: recommendations for the evaluation and use of gastrointestinal surgery to treat type 2 diabetes mellitus. Ann Surg 2010; 251: 399-405. 


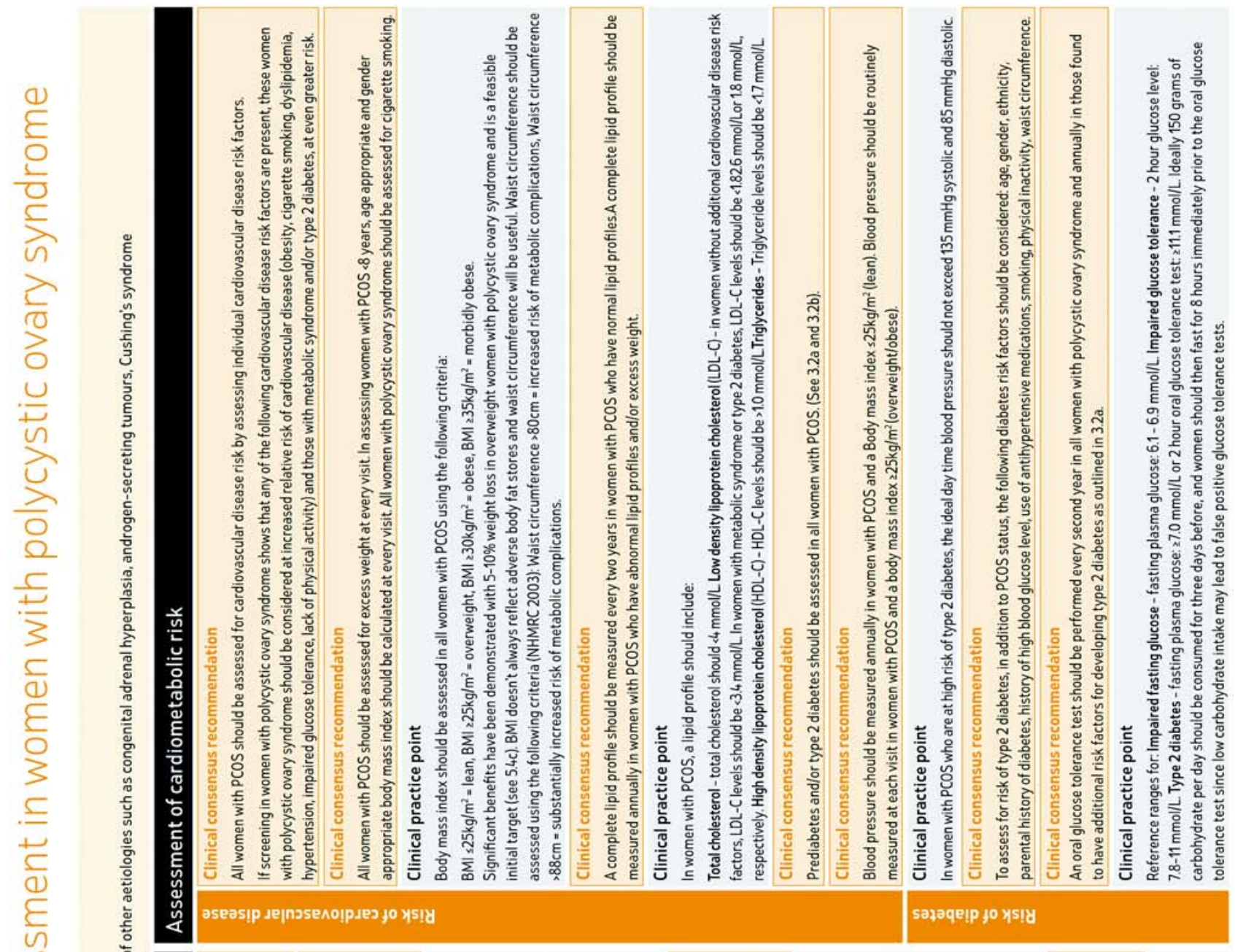

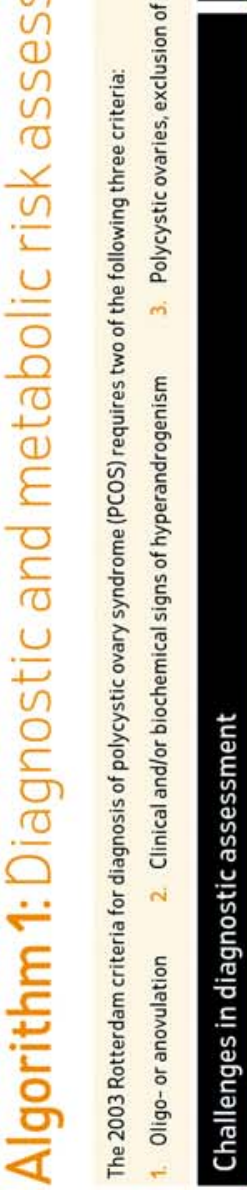

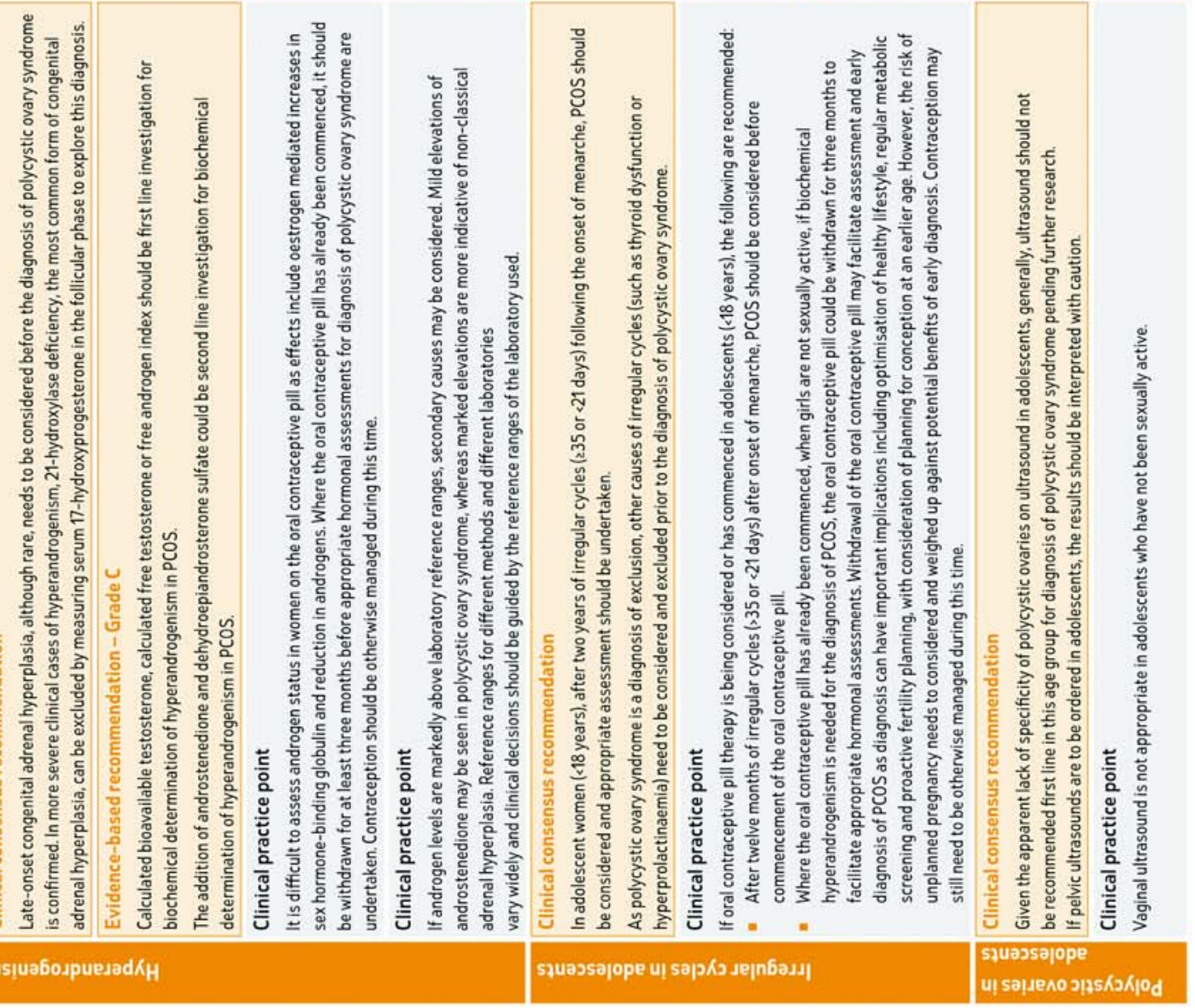




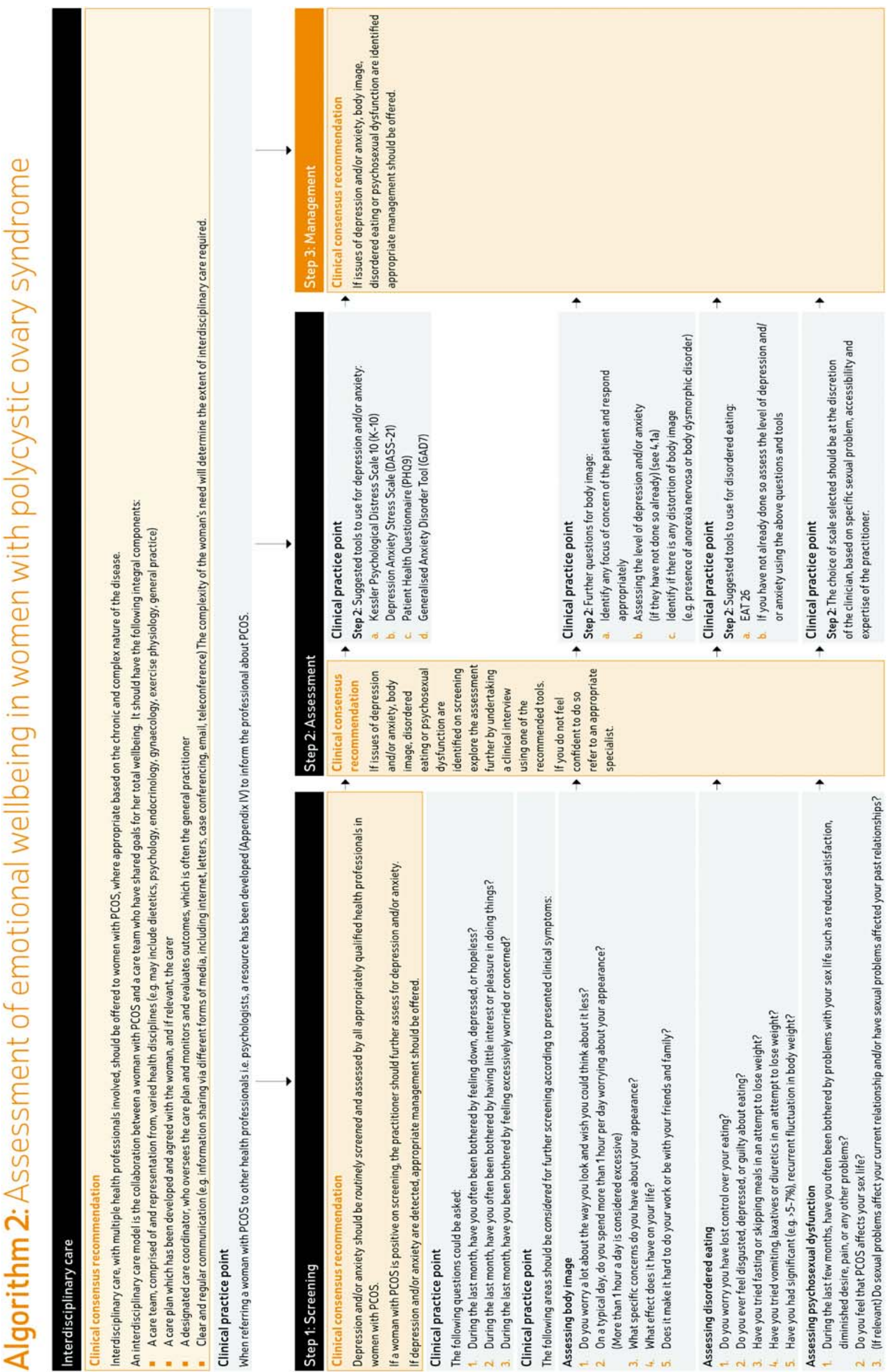

S108 MJA $195(6) \cdot 19$ September 2011 


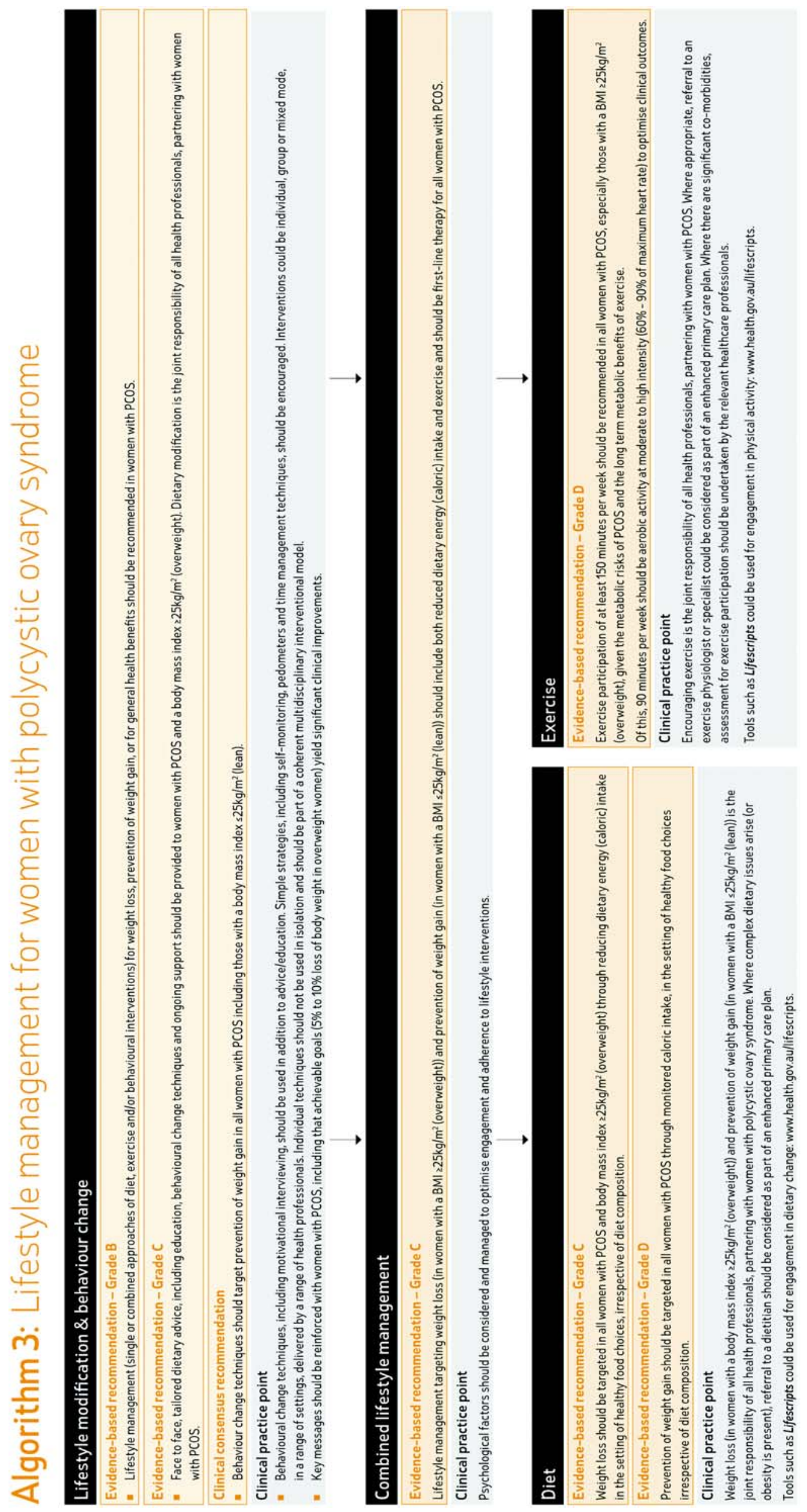




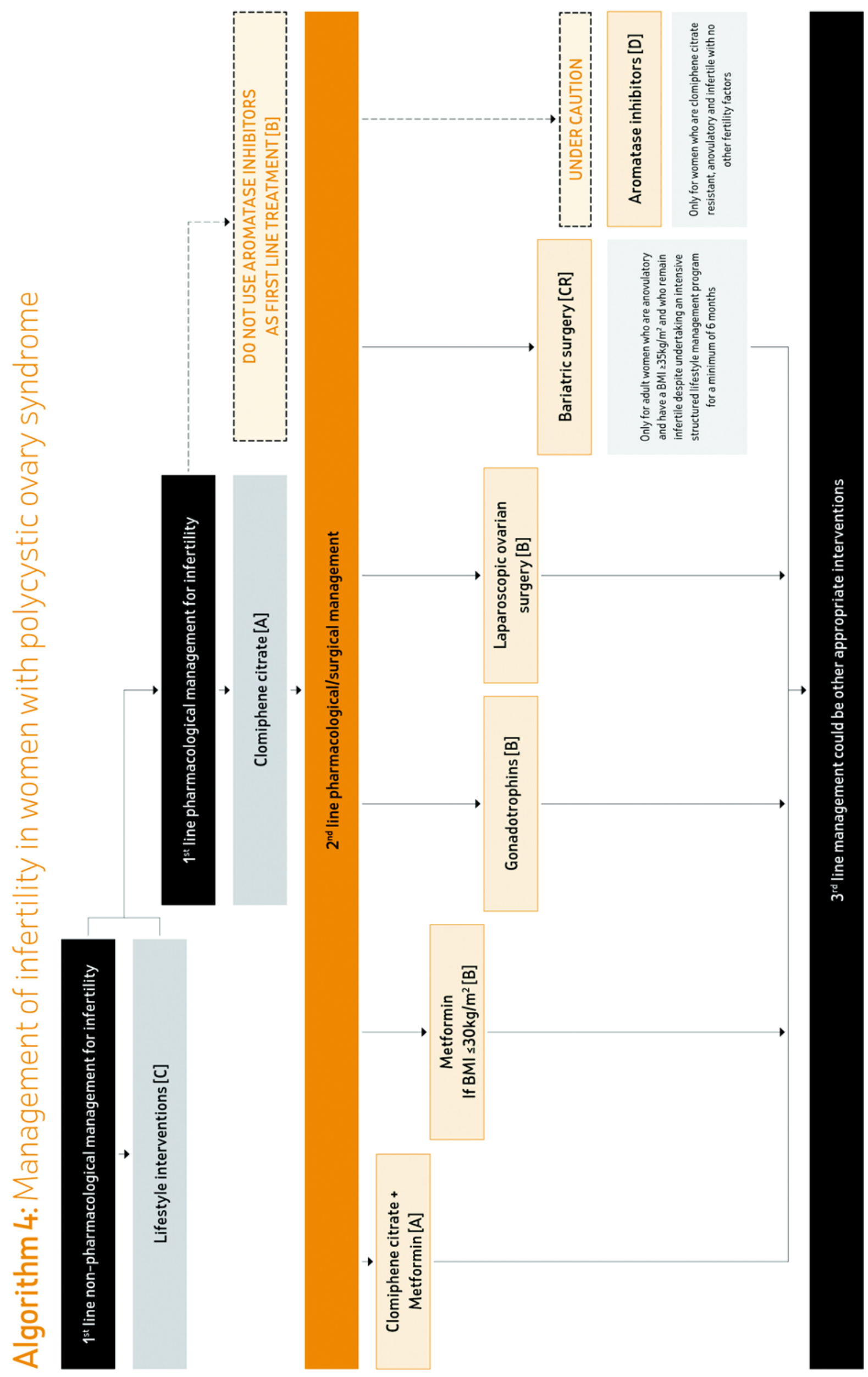

S110 MJA $195(6) \cdot 19$ September 2011 

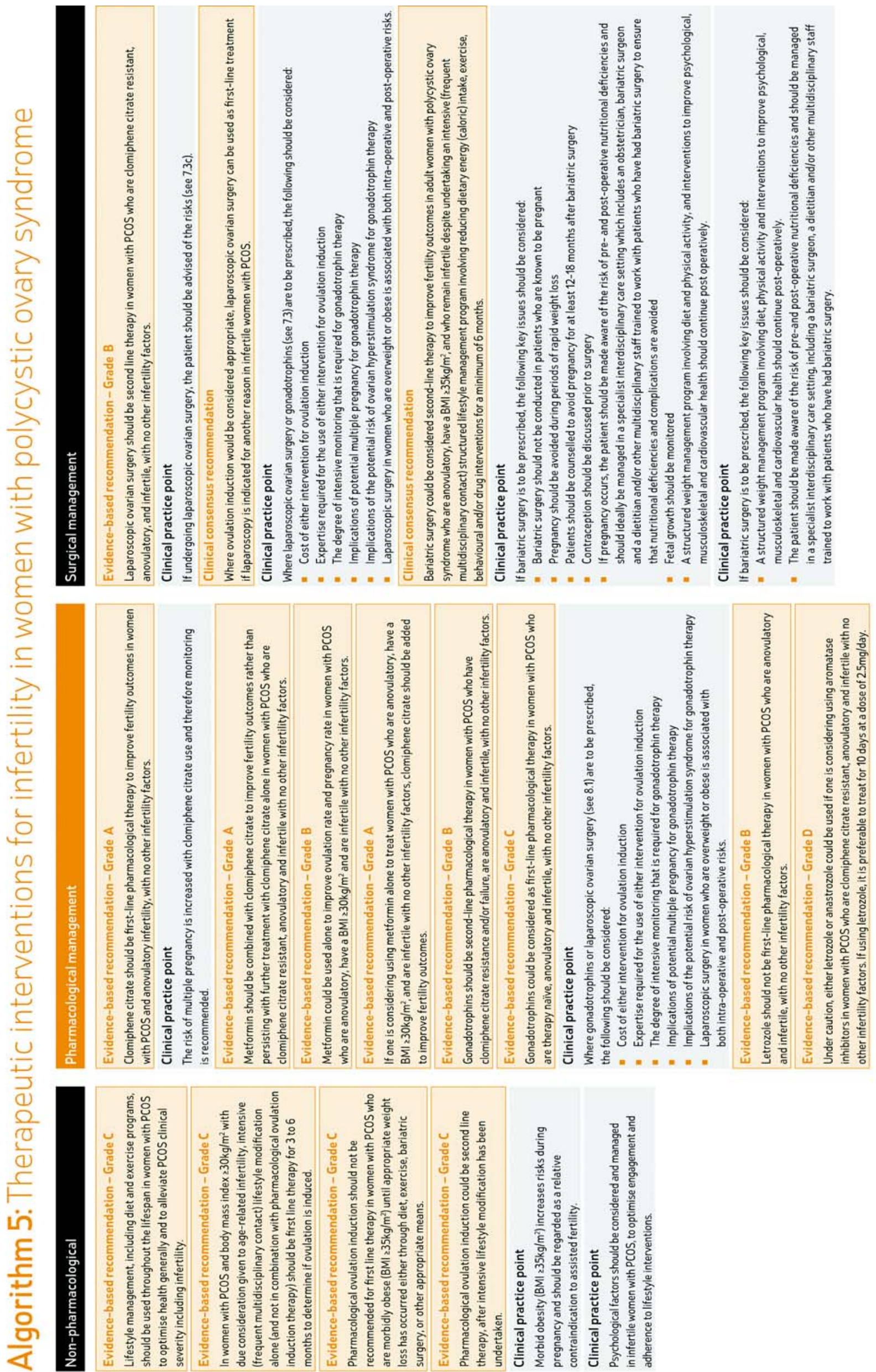


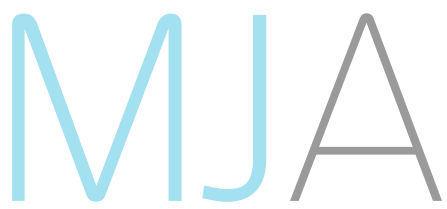

The Medical Journal of Australia

\section{Assessment and management of polycystic ovary syndrome: summary of an evidence-based guideline}

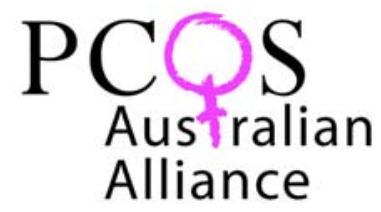

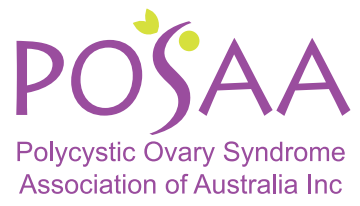

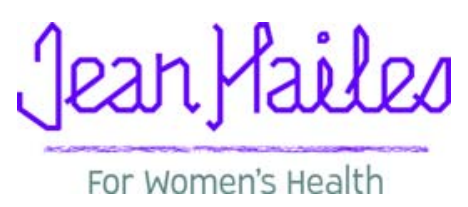

